
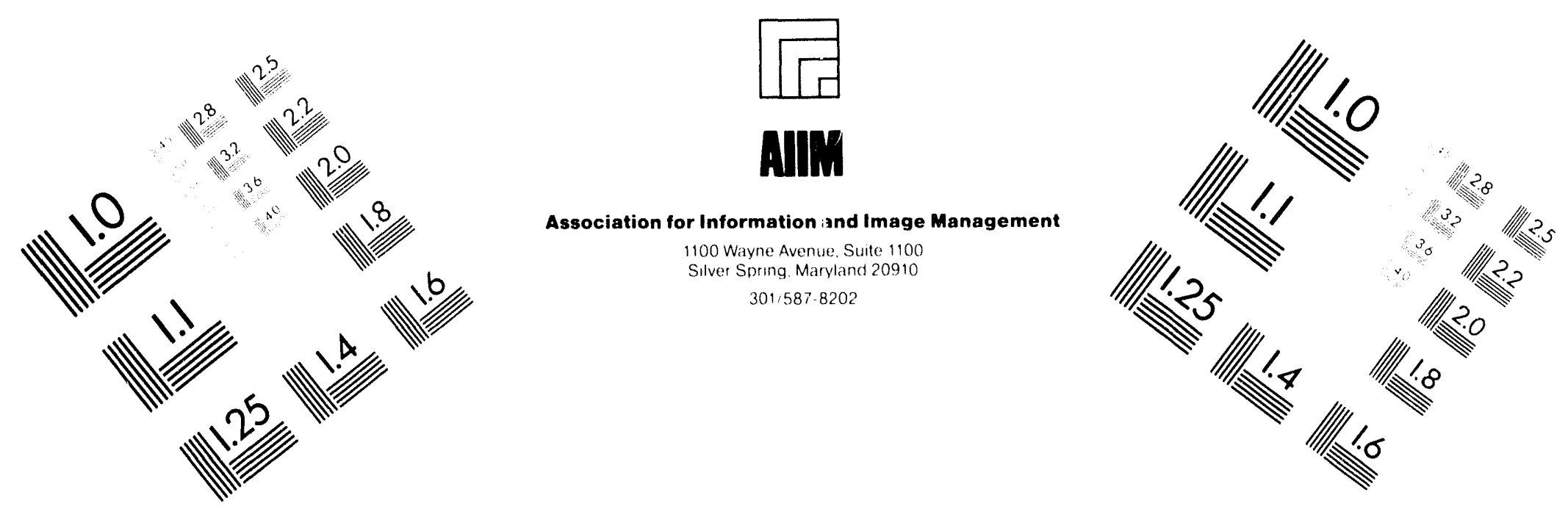

\title{
Centimeter
}

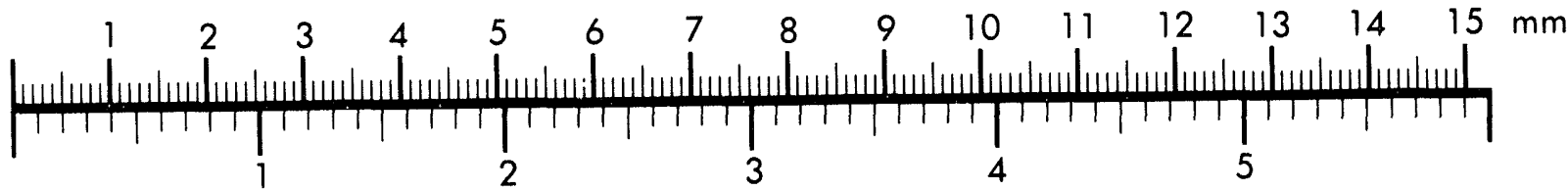
Inches
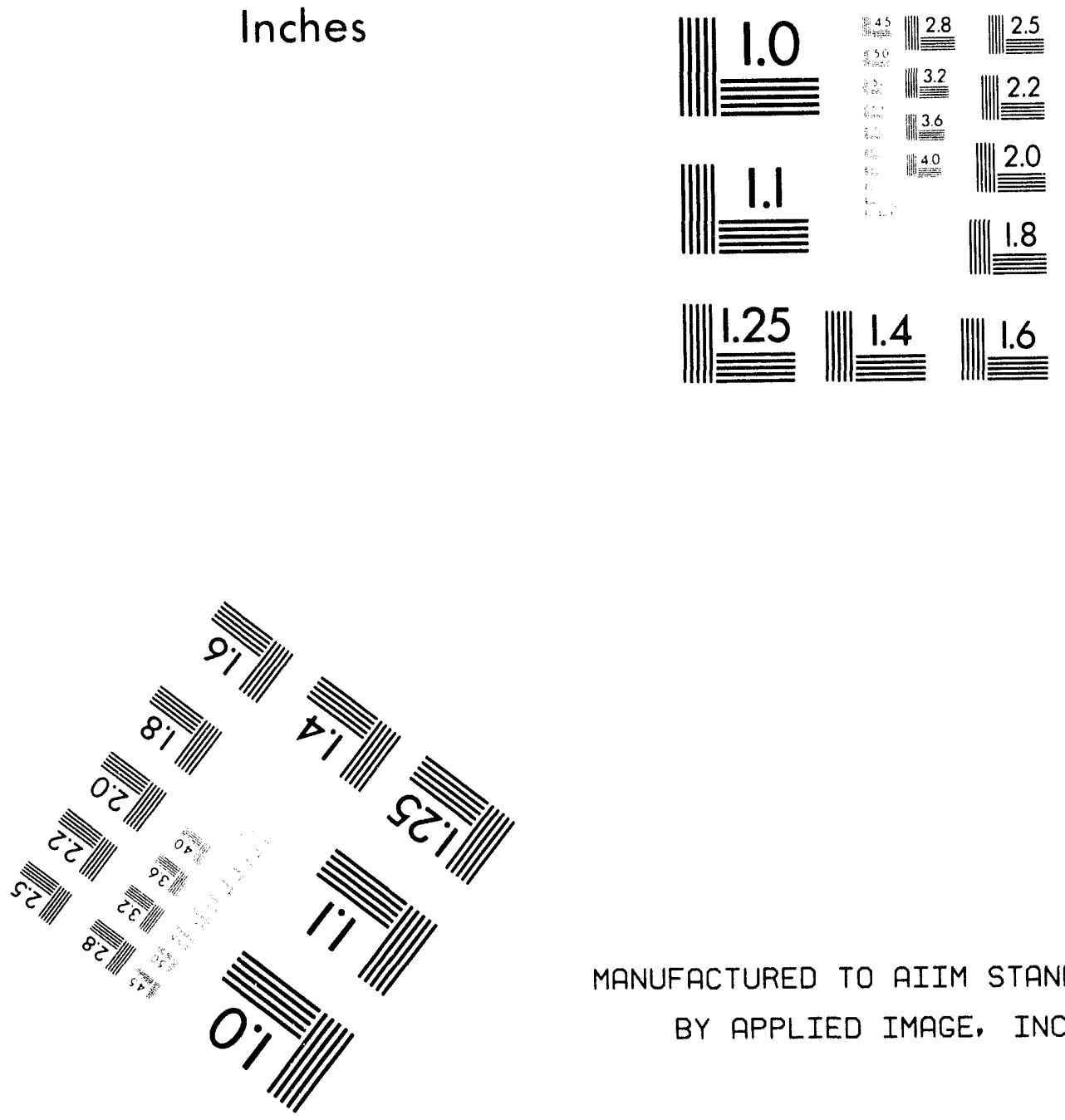

MANUFACTURED TO AIIM STANDARDS

BY APPLIED IMAGE, INC.

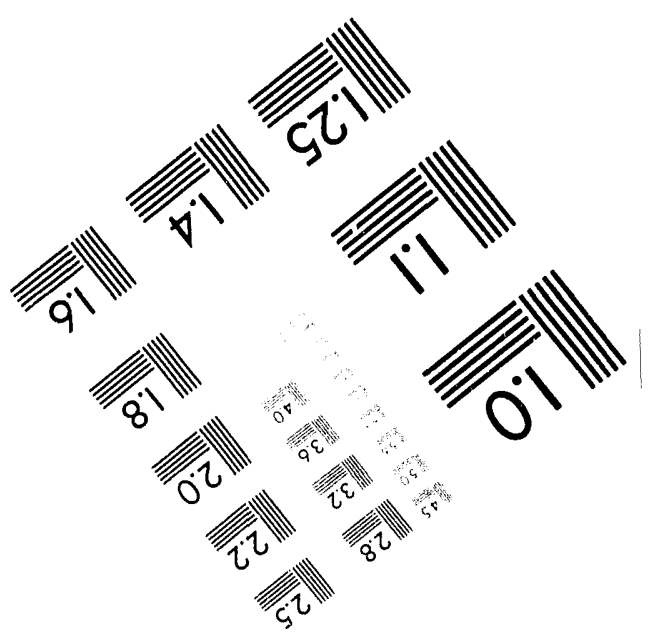



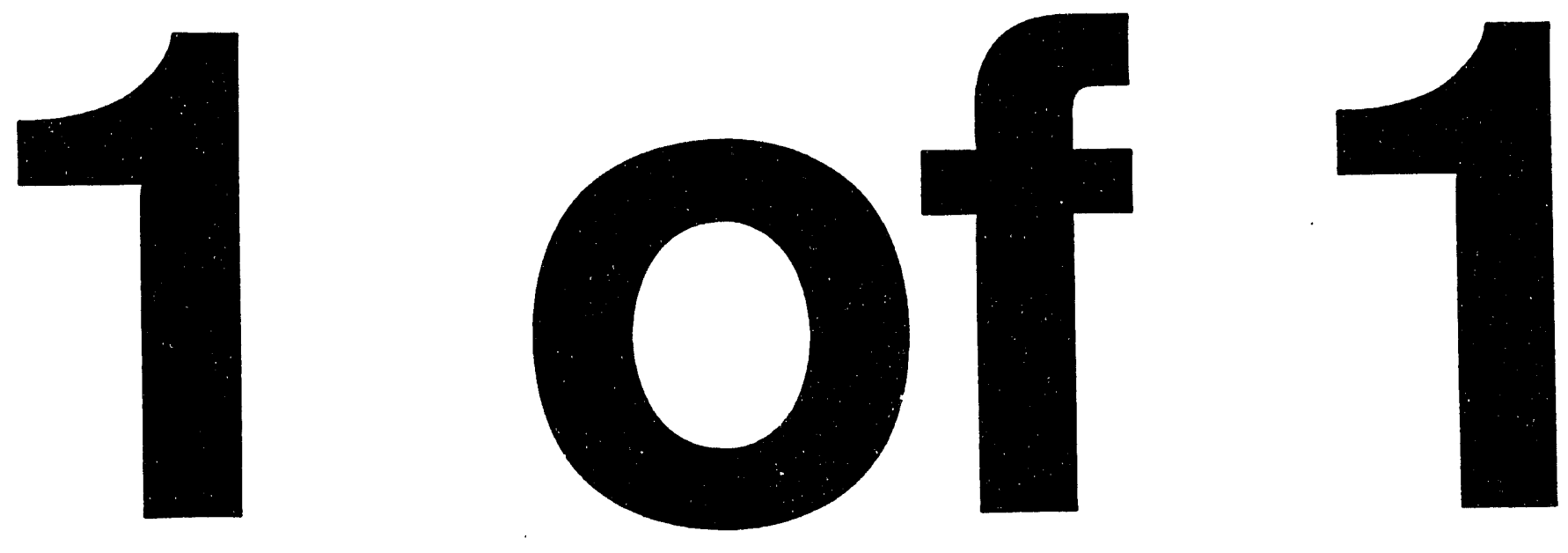


\section{Brm frotax}

2.

3.

4.

5.

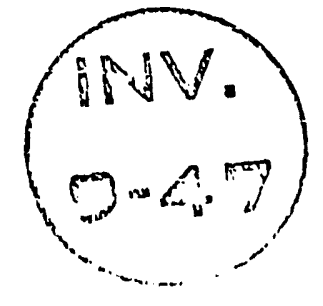

\section{$\%$}

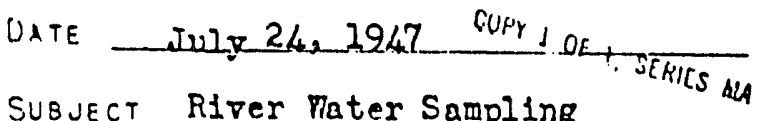

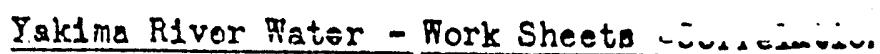
Tables

To F1lo

From B. F. Butler

\section{coov wo. + A}

BEFORE READING THIS DOCUMENT, SIGN AND DATF EELON:
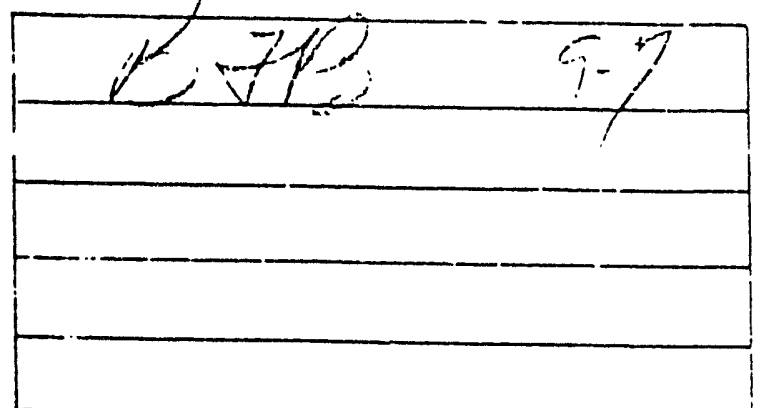

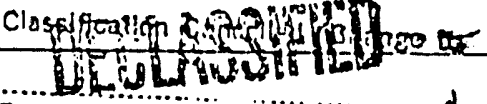

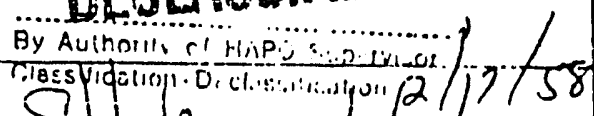

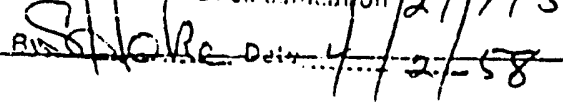

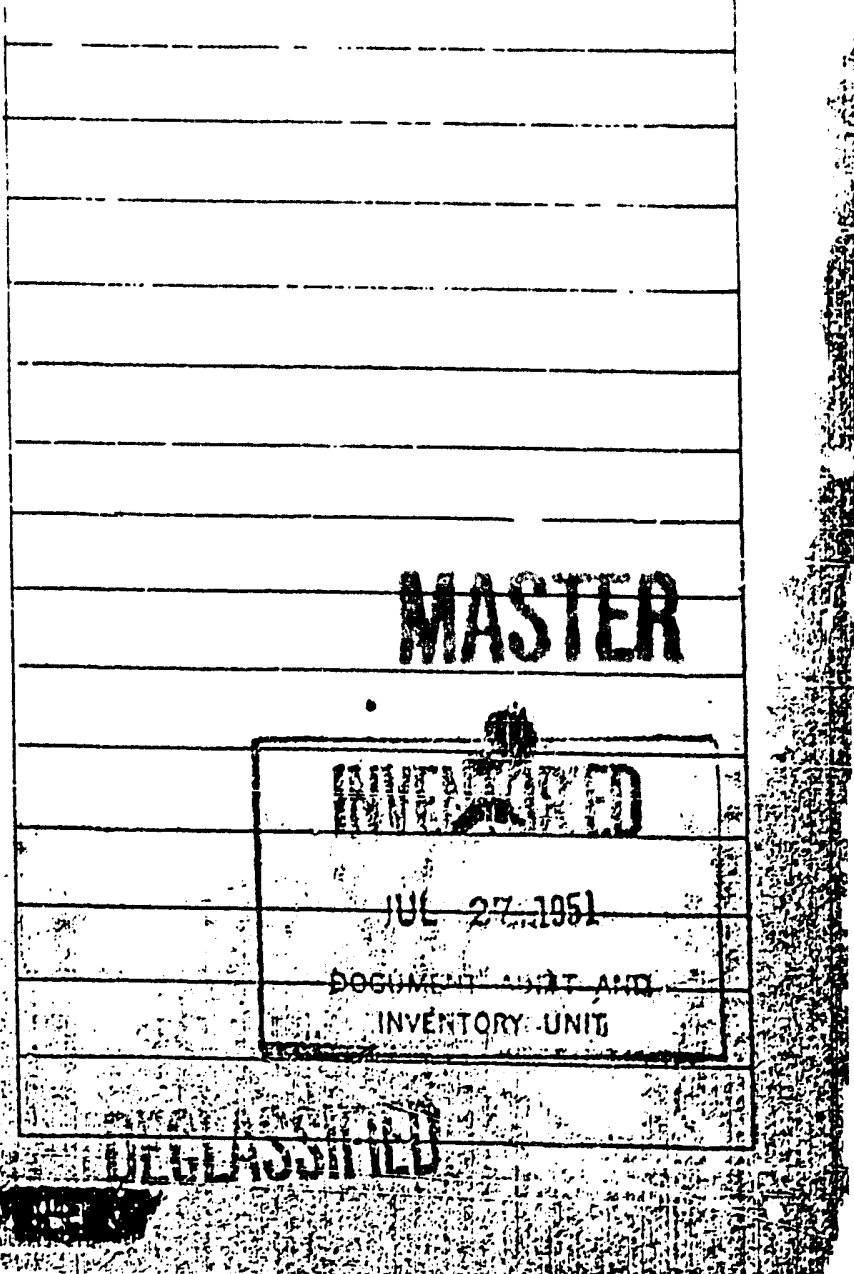
हit 6 


\section{DECLASSIFIEU}

chnchy

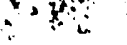

ENGINEERING DEPARTMENT

maser monyaturzia Piver Water

- Surtugeres tislex

II compurcin it Ale'ison

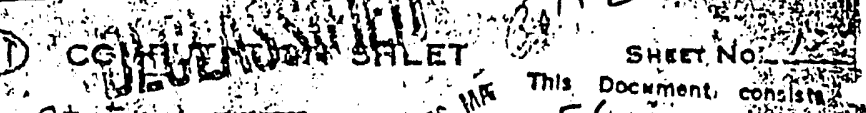

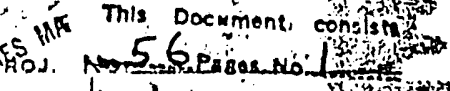

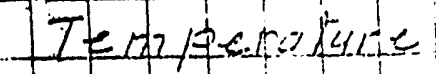

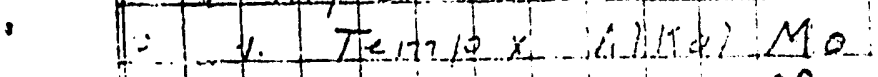

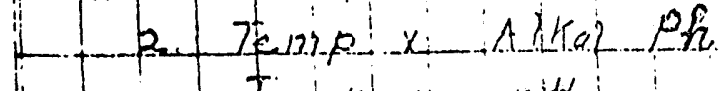

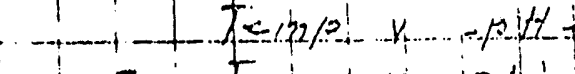

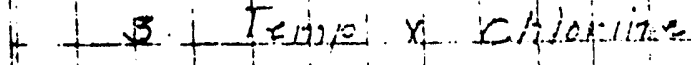

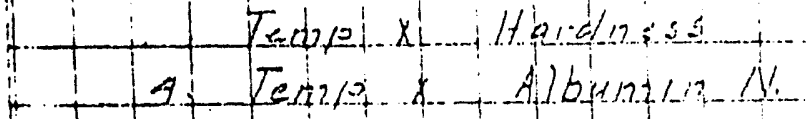

-........-

$\frac{1}{1}$

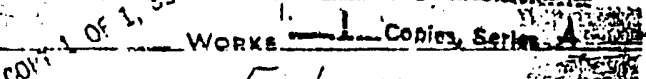

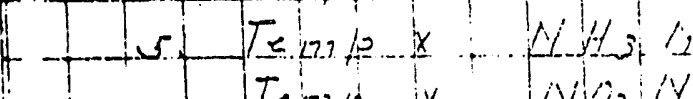

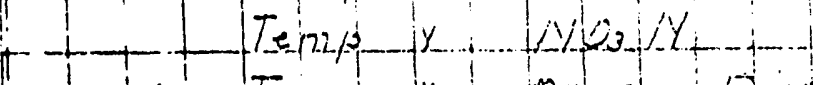

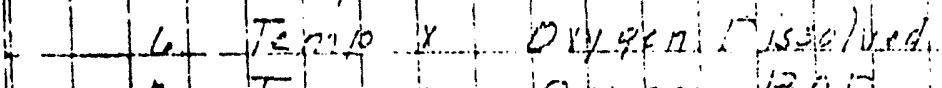

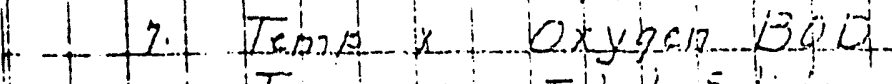

8.1 Tern ty: Thebielily

I.t. $+1+$

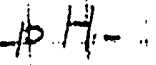

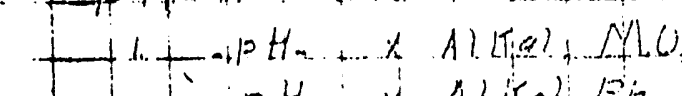

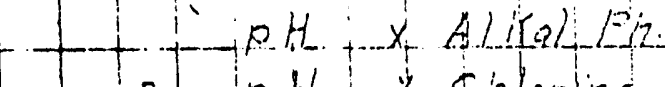

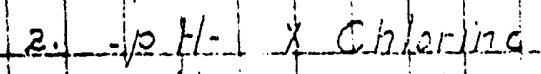

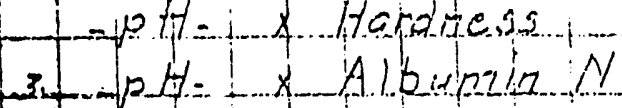

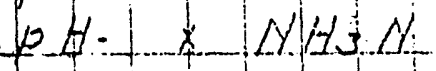

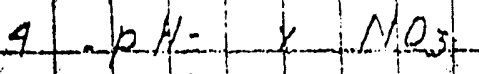

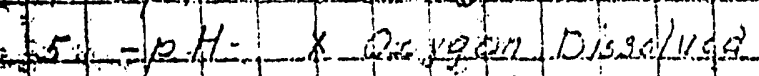

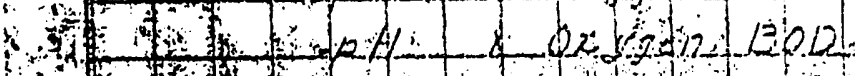

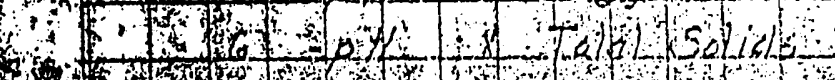

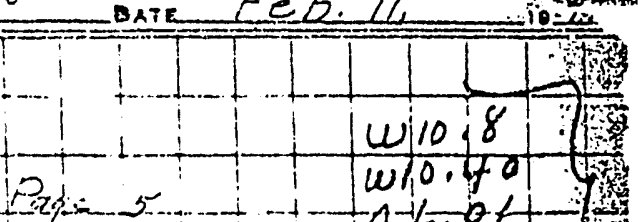

$A^{-1} 5.04-3$ $+A+6+(207)$

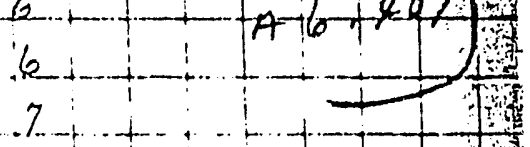

$2++1$
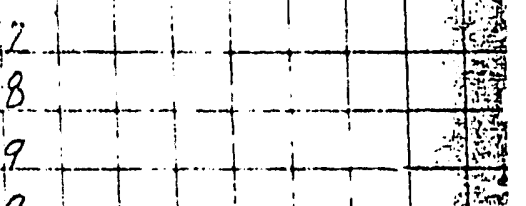

$9++\div+1+2$

10

11

is

+
+
+

in

ㄴ.

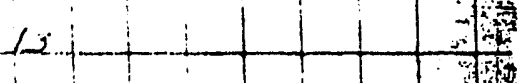

13

11.

$19 .+$

int

36

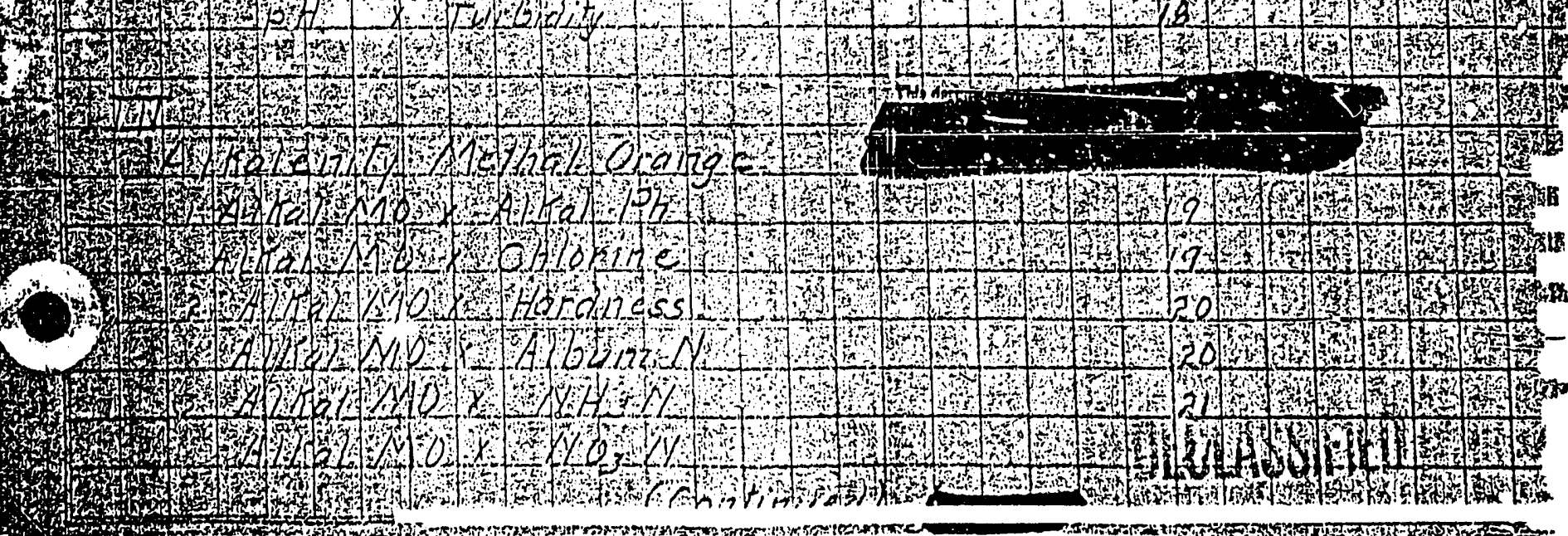

DECLASSIFIED 
$\therefore$ DECLASSIFIED

ENGINEERING DEPARTAENT JUPUID ${ }^{2}$ COMWUTATION SHEET

HQ

TITLE of pros

LIICLEz

PROJ. NO

SHEET NO

Worko

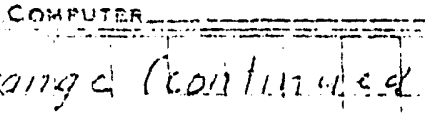

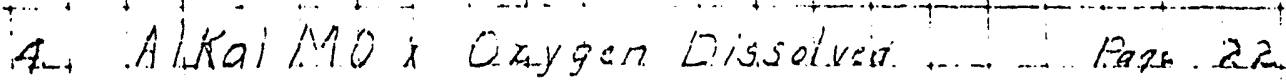

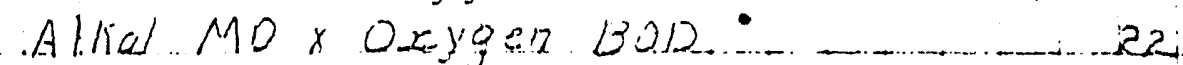

.5. Alkal MO x Tolal. Solia's

Alikal. Mo x Titrbidity.

2.3.

IV.

- Aikolenity Phenoiphthalfin

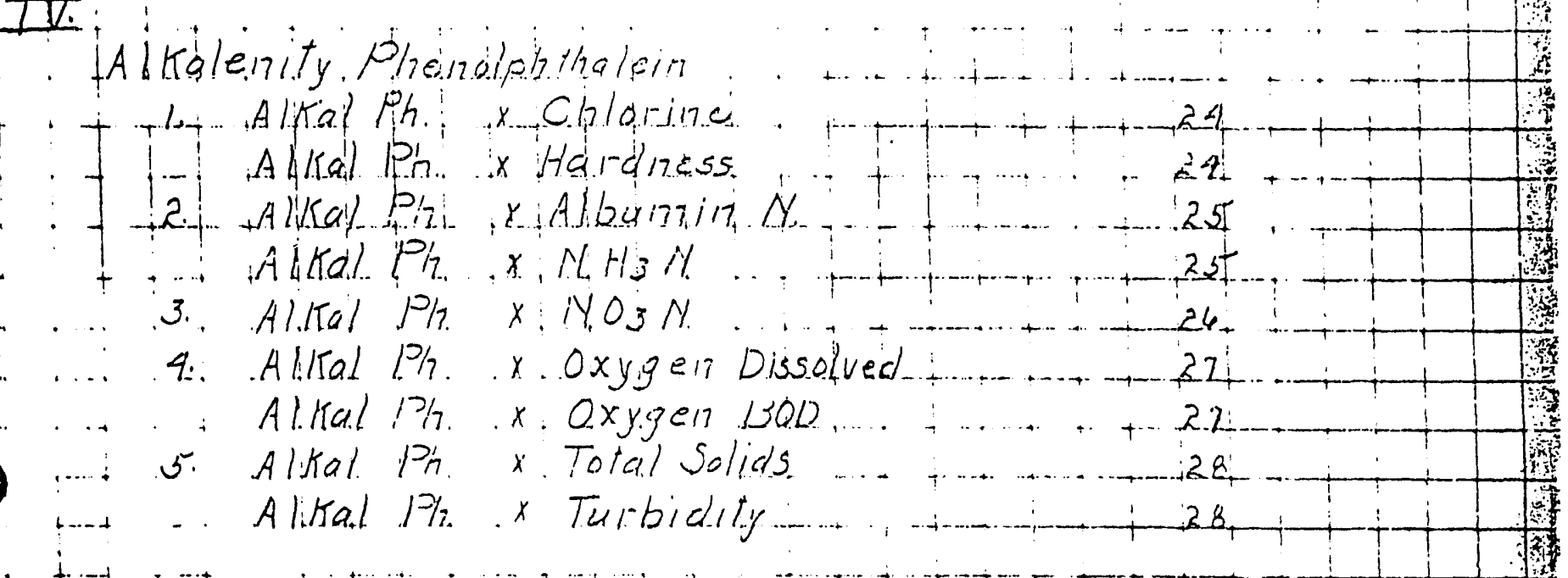

Ditosine

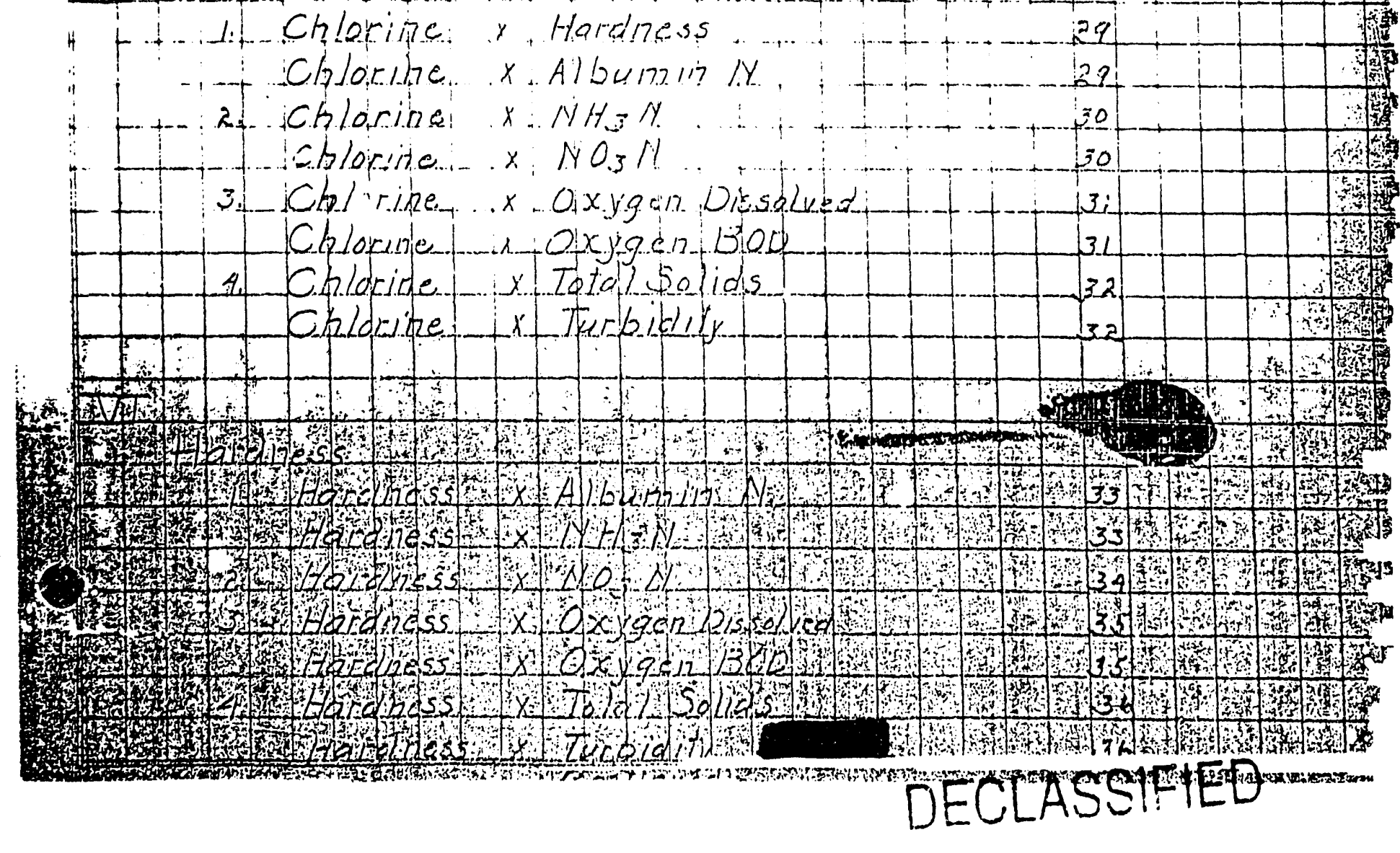




\section{DECLASSIFIED

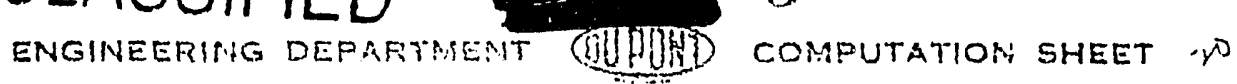 $l y d c . x$ \\ MOHKE \\ DATE 3}

susuect

VI.

A L bumpuaich Nitroger.

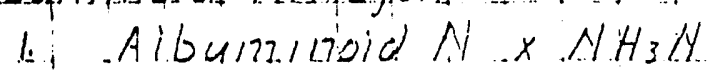

Albumziliz. N

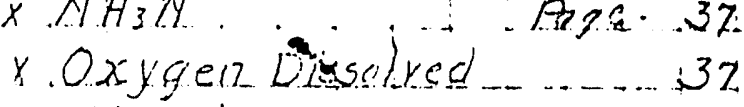

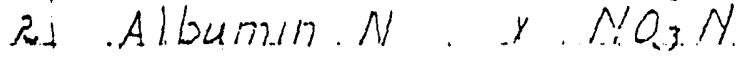

32

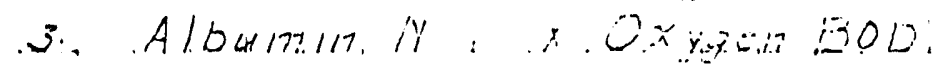

39

$\therefore$ Albums N A Turbidily

139

- $\quad+\quad+$

VETIT

$\mathrm{NH}_{3} N$

. $\mathrm{NH}_{3} \mathrm{~N}, \mathrm{~N}, \mathrm{NO}_{3} \mathrm{~N}$

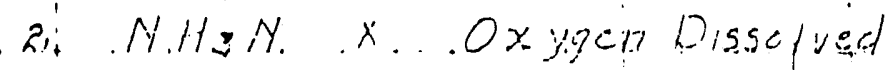

NHizil $\times$ Oxygen Boo

40

3. MYtiziti X. Total Solicis.

MHzh \% Turbirily

HI:

42

12.

23

12

Ix.

Mes 11

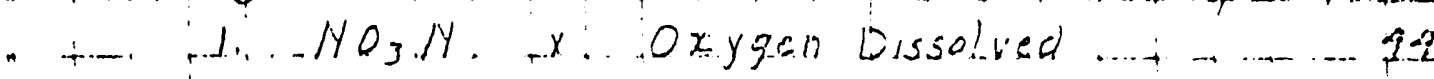

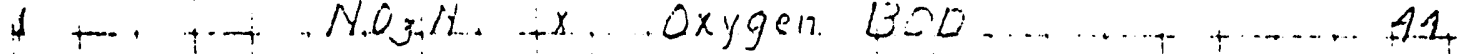

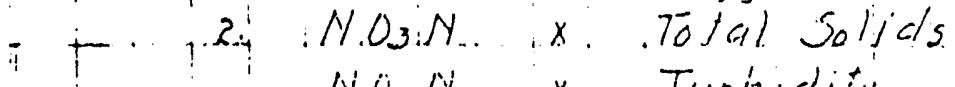

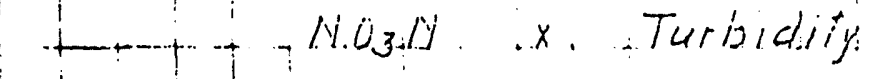

45

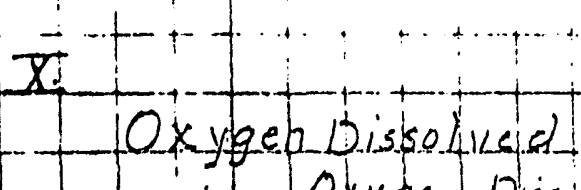

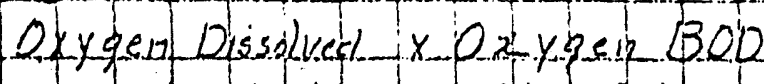

Oxygen Dissolvece $x$ Taltial Solids

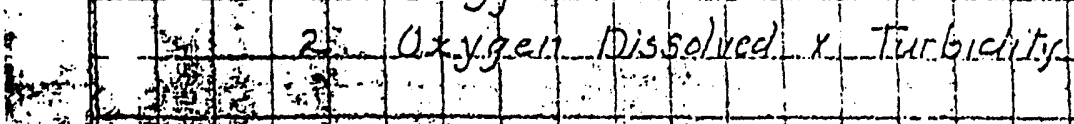

\section{-}

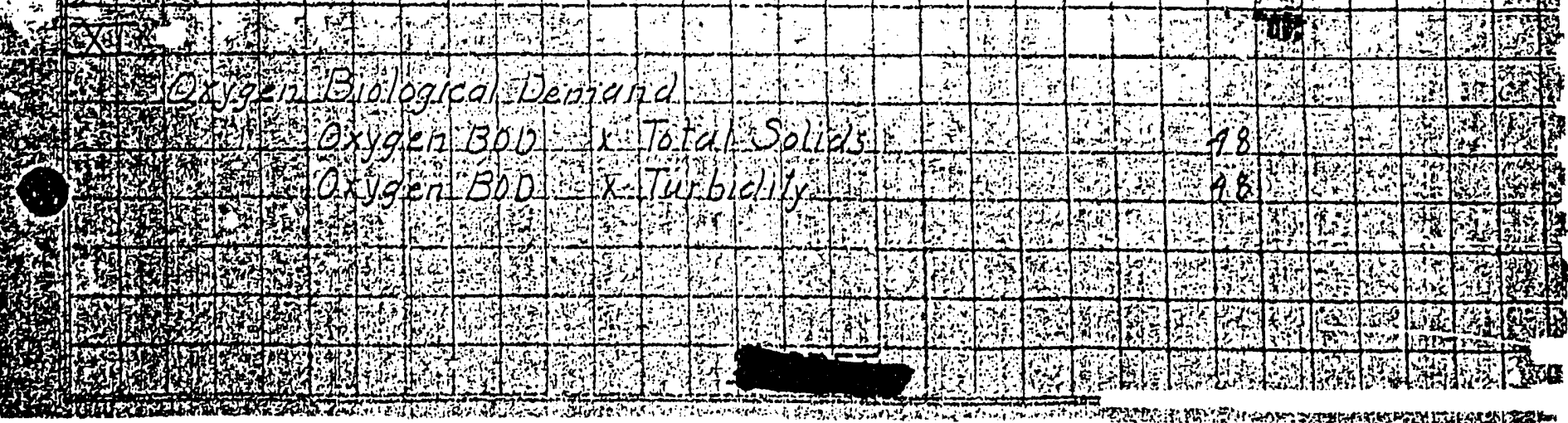

DECLASSIFIFח 


\section{DECLASSIFIED}

$\rightarrow-\infty$

ENGINEERIMG DEFARTMENT (IIU⿴囗WD

lindex

Tine of prod.

Busjzer

XII.

Total Solids

i. Th Totali Solids x.. Turbidity

Pags: 79

$x \sin$

Mostly of year.

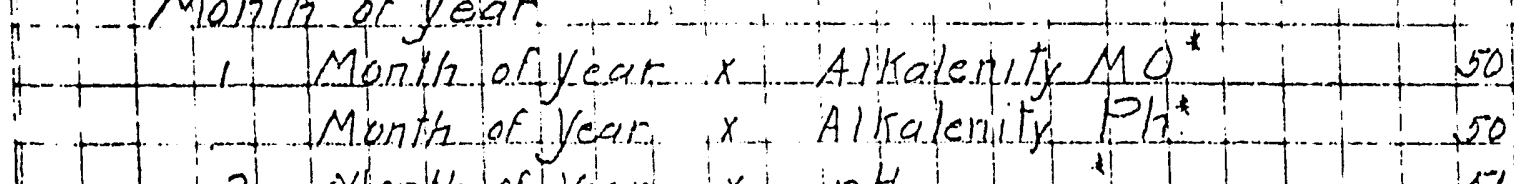

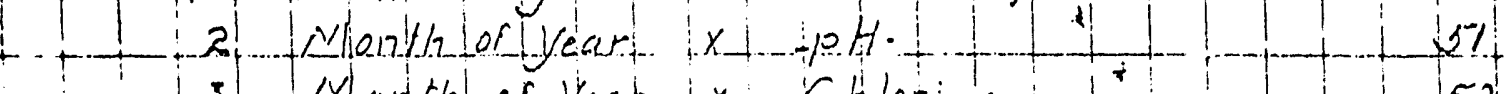

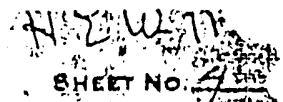

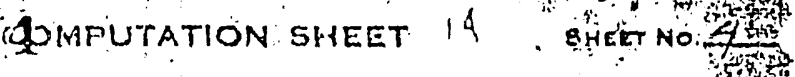

PROJ. No

, i.

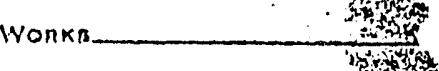

t +

53

ing

Month af year $x$. Hardizoss

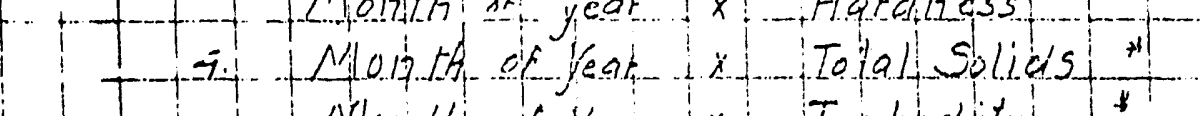

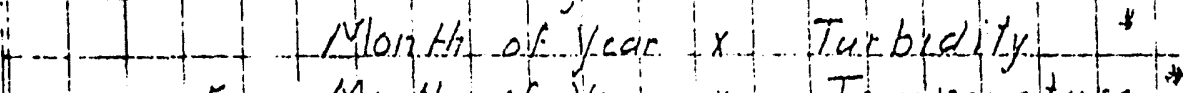

5

Month of yea x x Temperatule.

522

$1+1.1 \frac{19}{4}$

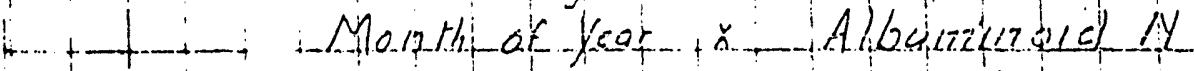

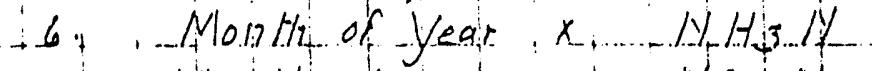

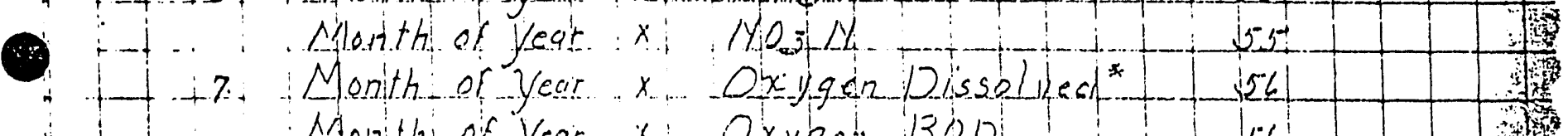

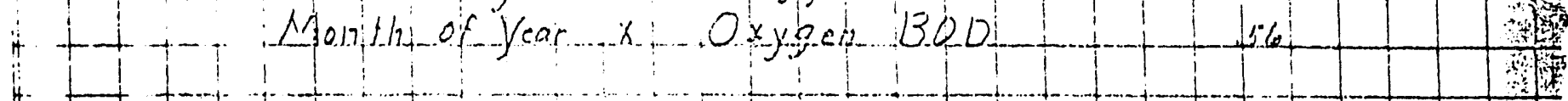

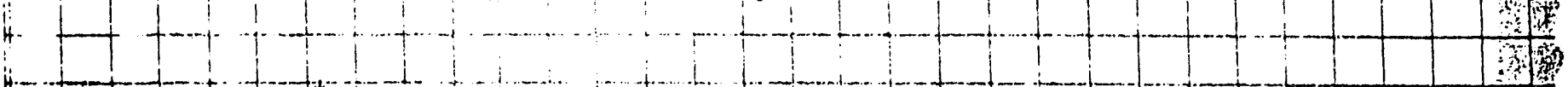

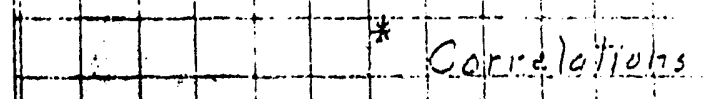

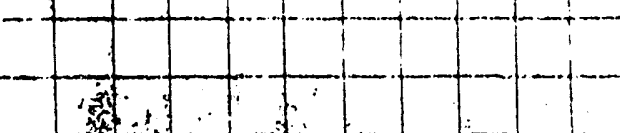

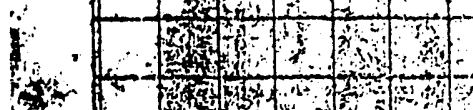

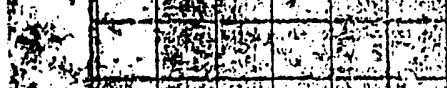

sots

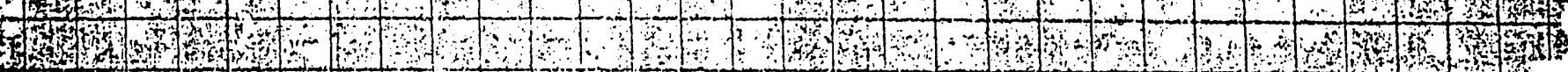
7. 30 (3)

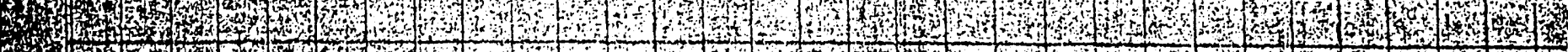
3
4

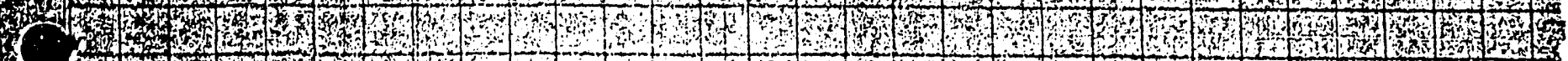

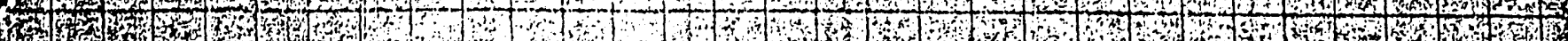
4 (s) s.

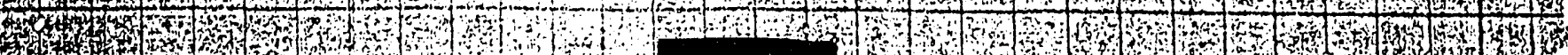

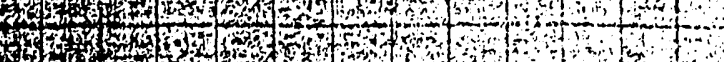

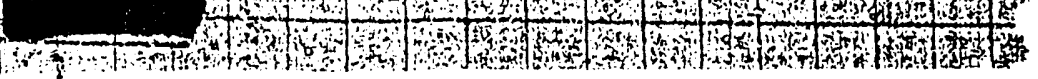
DECLASSIFIED 
PROJ. NO sity

\section{in 2}

TitLE or PROS.

Stethin $\sqrt{11}$

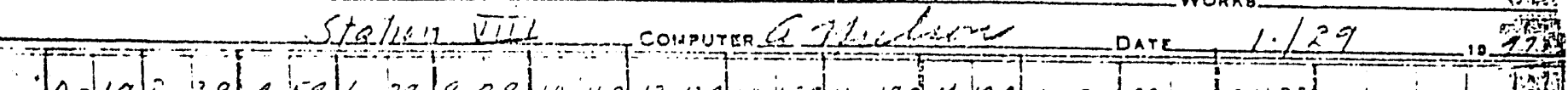
WÓR $\times 8$

ing

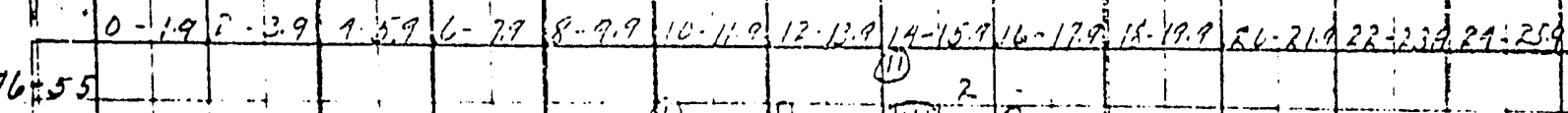

$56 \div 5$

46475

$26.85+\ldots+$ (III)

8495

$6+1$

.

(i) 15

(III) 2 -

(106155

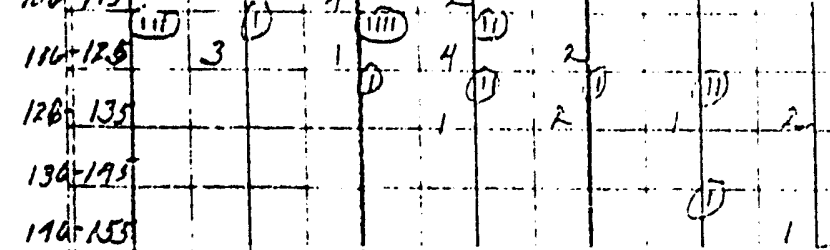

154165

$-16405$
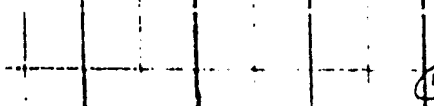

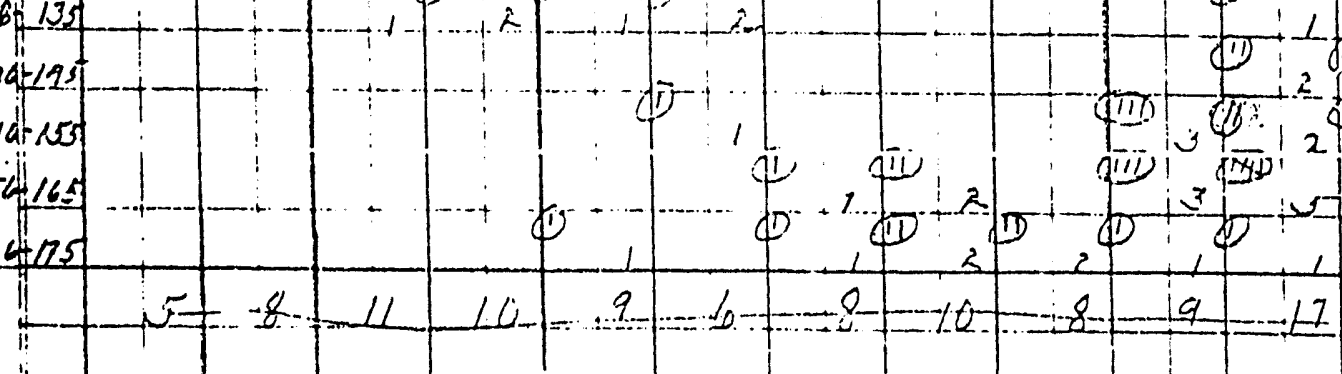

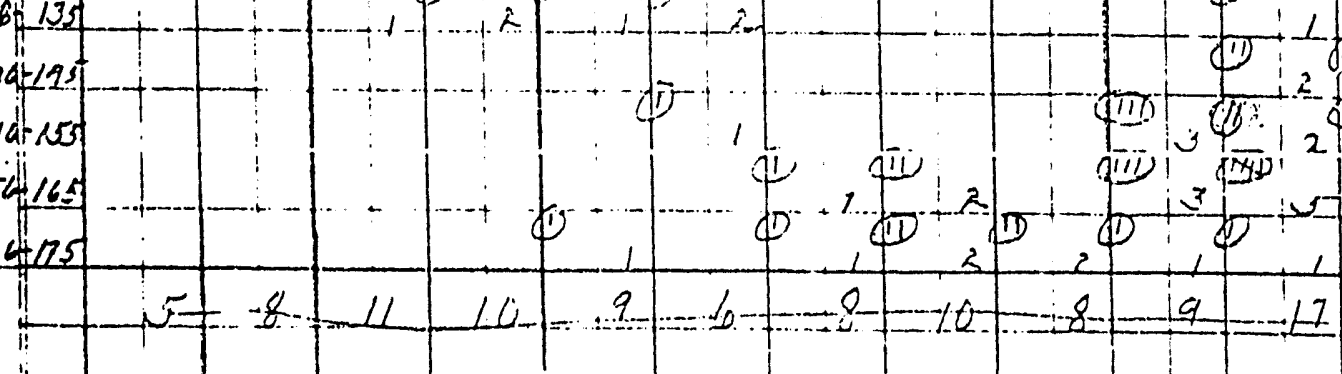

(ii) ${ }^{2}$

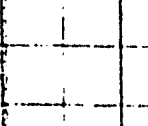

MII

2: $\quad \frac{1}{214}$

$0+5$

1129

\begin{tabular}{l|l|l}
$-i$ & $j$ & $j$ \\
4 & $j$
\end{tabular}

$+7$

1

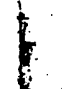

1: $\quad+4$

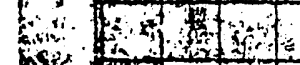

s.

(1)

(6)

3.

Th

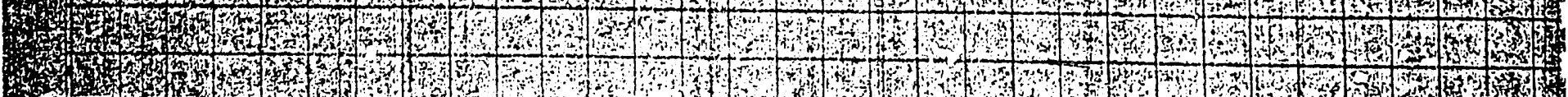

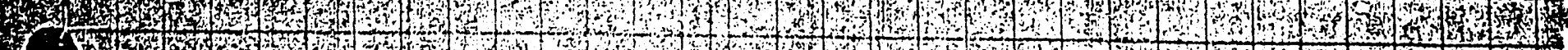

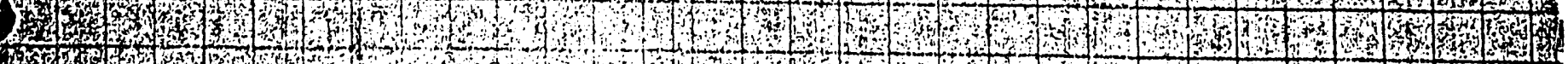

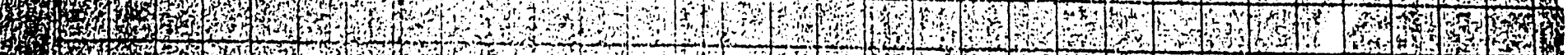
675 (x)

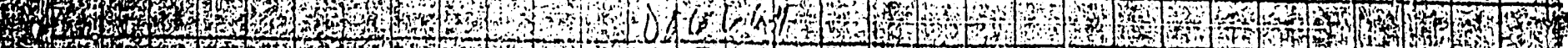
- 7 . 
TITL or PAOS

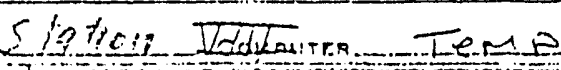

WORK:

SUEjeCt

-

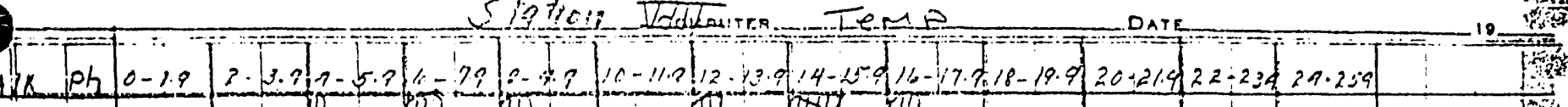
a-1.9 (11) (II)

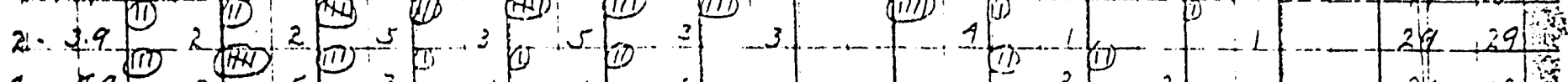

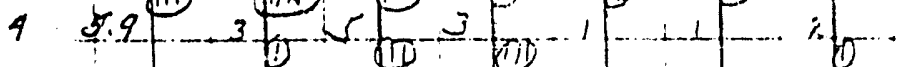

$6=1: 9$

$8=.9 .9$

$10-4 \cdot 9$

$12-13.9$

$1.2-12.9$

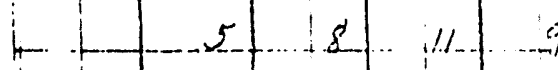

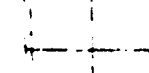

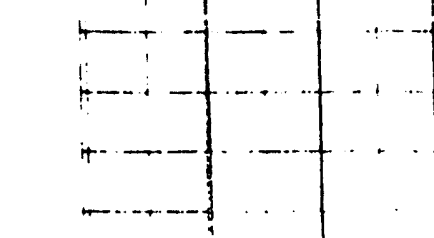

Tello The olf

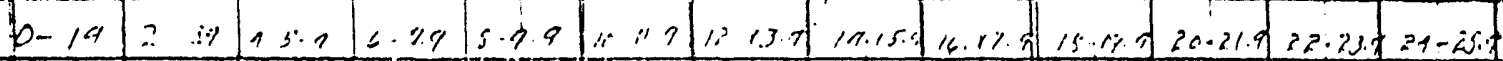

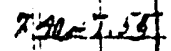

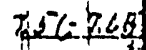 \\ Picle-270}

$2.26-2.89$

thergas

$20 b=28$.

\$26- 8.16

$\therefore \quad 860$

as
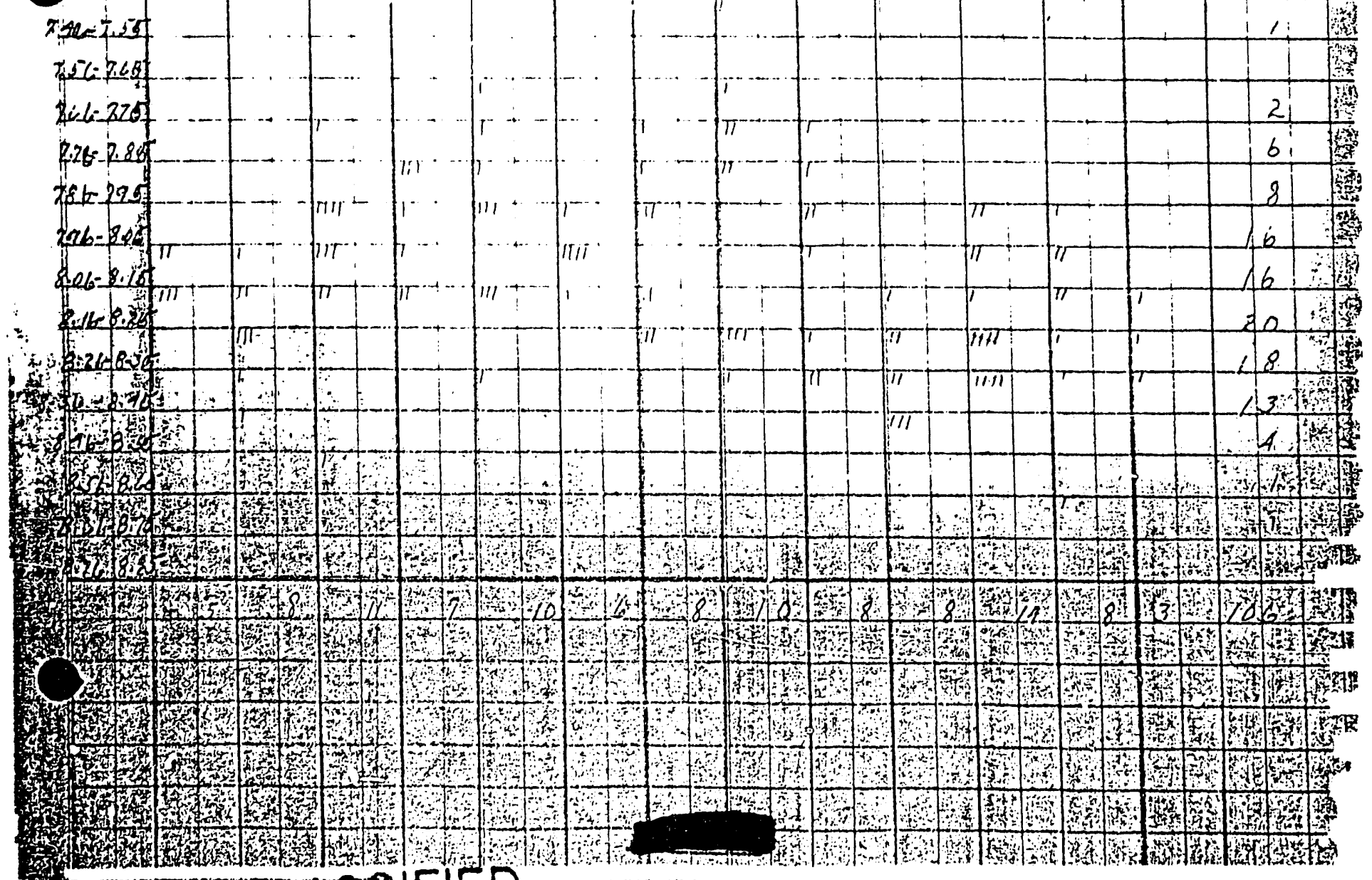

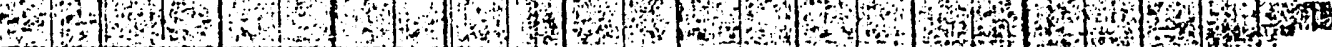

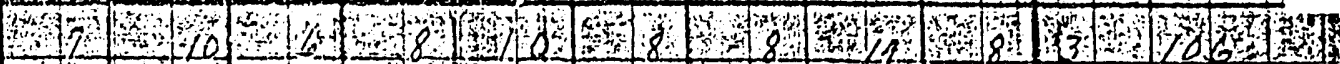

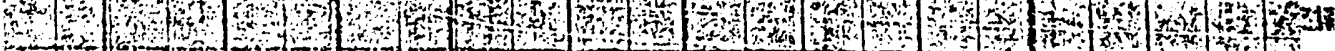

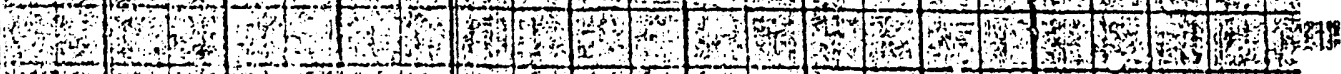

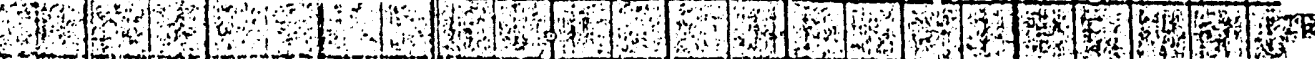
(2)

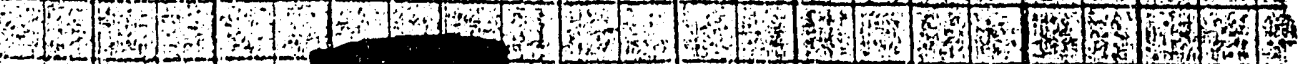

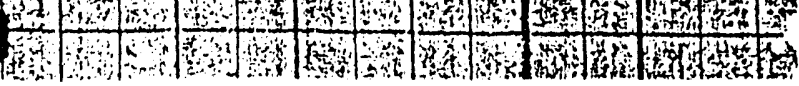


TITLE of PROS

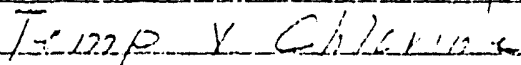
Wอกหร

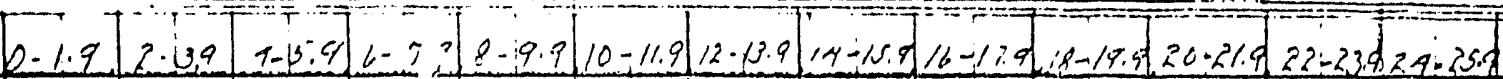

$\therefore 1-.5$.

6.60

$121-1.2$

$1.6-2.0$

$3.1 \cdot 2.57+11$

$34-3.4$

T-

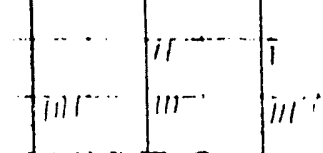

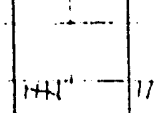

$9.6-9.0$

$0.1-1.5$

$1.6 \cdot 5.00$

Si: $1=5 ;$

$12 \quad 8979.6$

9.

9

-.......

-..

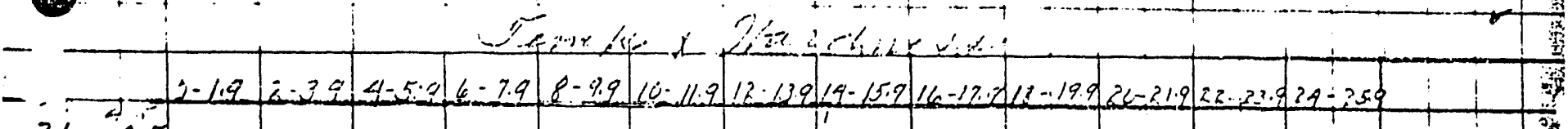

$34-25$

$4 k=5=5$

उद 5 पर

$4 k-175$

$76=85.7 r^{-\ldots i l l}$

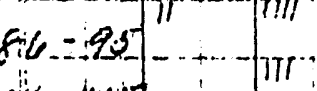

$9 \%=10.37$

atherilis

itlectrata

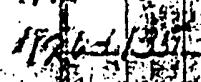

mbing

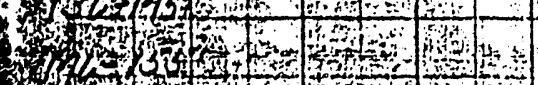

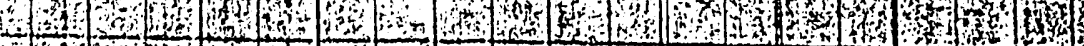

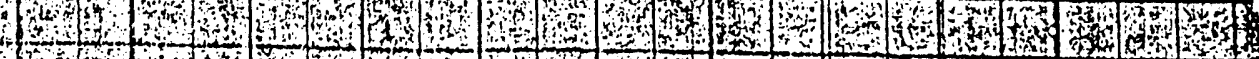

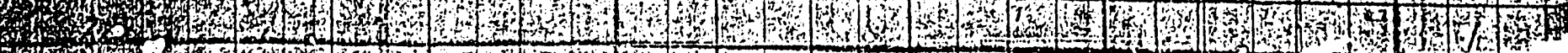

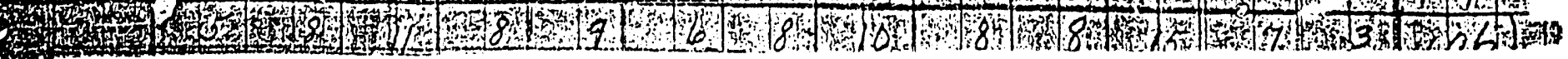


ENGINEERING DEPAFTMENT OUIPOHD CONBUTATION SHEET

TITLE or PROS. DPROS No

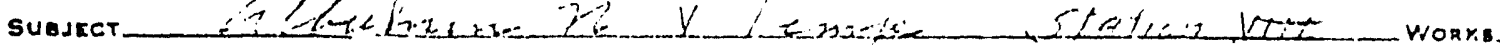

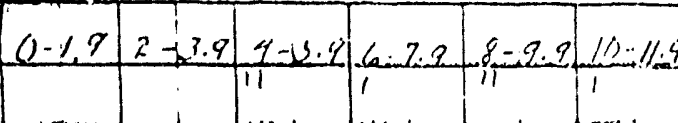

ousuren.

$.01-q \geq q$

$.0 \$-.049$

$-.069$

$07-.089$

.49-109

$.11=429$

$13-140$

$15-140$

$1 \mathbb{R}-189$

$+19-1209$

-2t- 22

$.23-24 t$

- $85.5^{2}<4$

$.22-.289$

24-304

$-31-328$

33-31.

ii

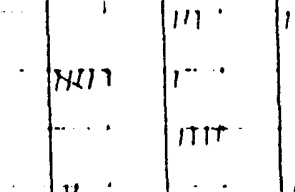

... .

......... 


\section{-.... DECLASSIFIED}

ran. or ono,

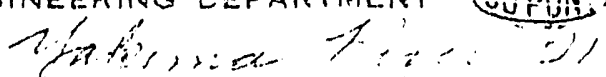

Zomputation sheet

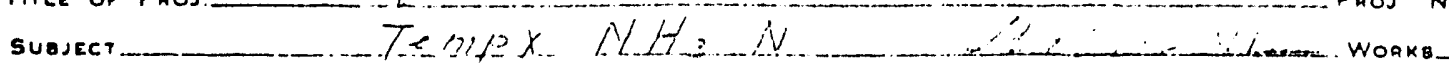

cosputen

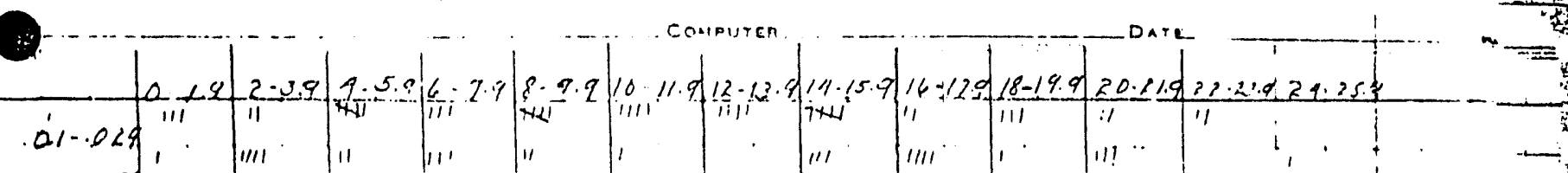

.03 .099

ar isit

$67 \cdot 689$

$09 \cdot \cdot 104$

.1]. .1?

$.13-.1 .99$

$.15-.169$

$.17-.189$

$.18-209$

, in

..

.

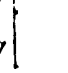

\begin{tabular}{|l|l|l|l|l|}
\hline & & & \\
\hline 3 & 8 & 11 & 8
\end{tabular}

\begin{tabular}{l|l|l}
\hline 9 & 6 & 8
\end{tabular}

\begin{tabular}{l|l|l|}
10 & 8 & 8
\end{tabular}

16

6

3.146

$-$

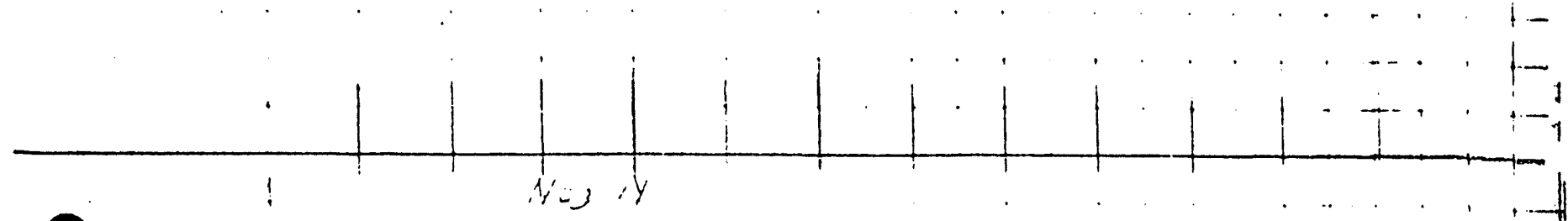

$\theta$$$
.01-.027
$$

$=3 \cdot .049$

.0 .5 .0 .69

$.07 \cdot 0.04$

$.09-\left..109\right|_{1} ^{.11-.179}$

$\therefore .13 .-199$

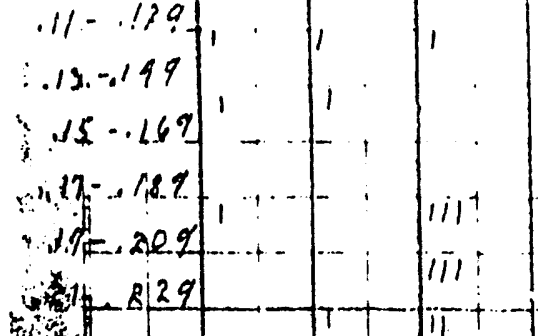

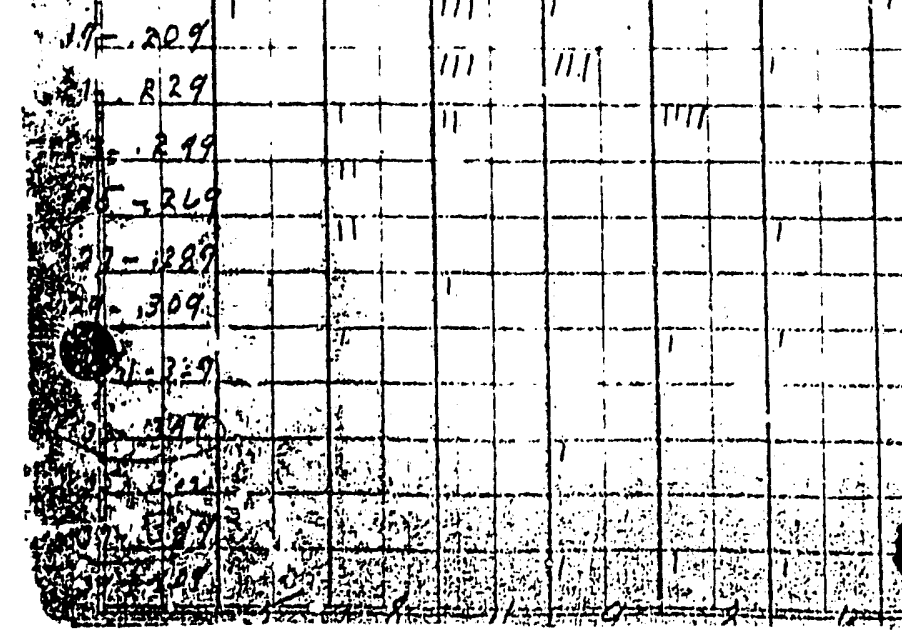


onm in. 41

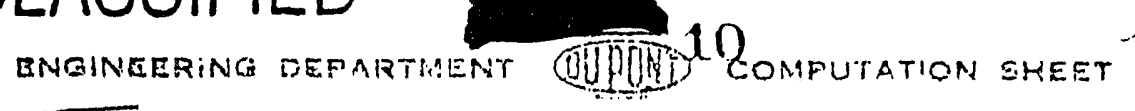
Hi $\bar{\omega} \omega \cdot \mathrm{j}^{2}$ Tirke or pros. IeNP X $X .0$

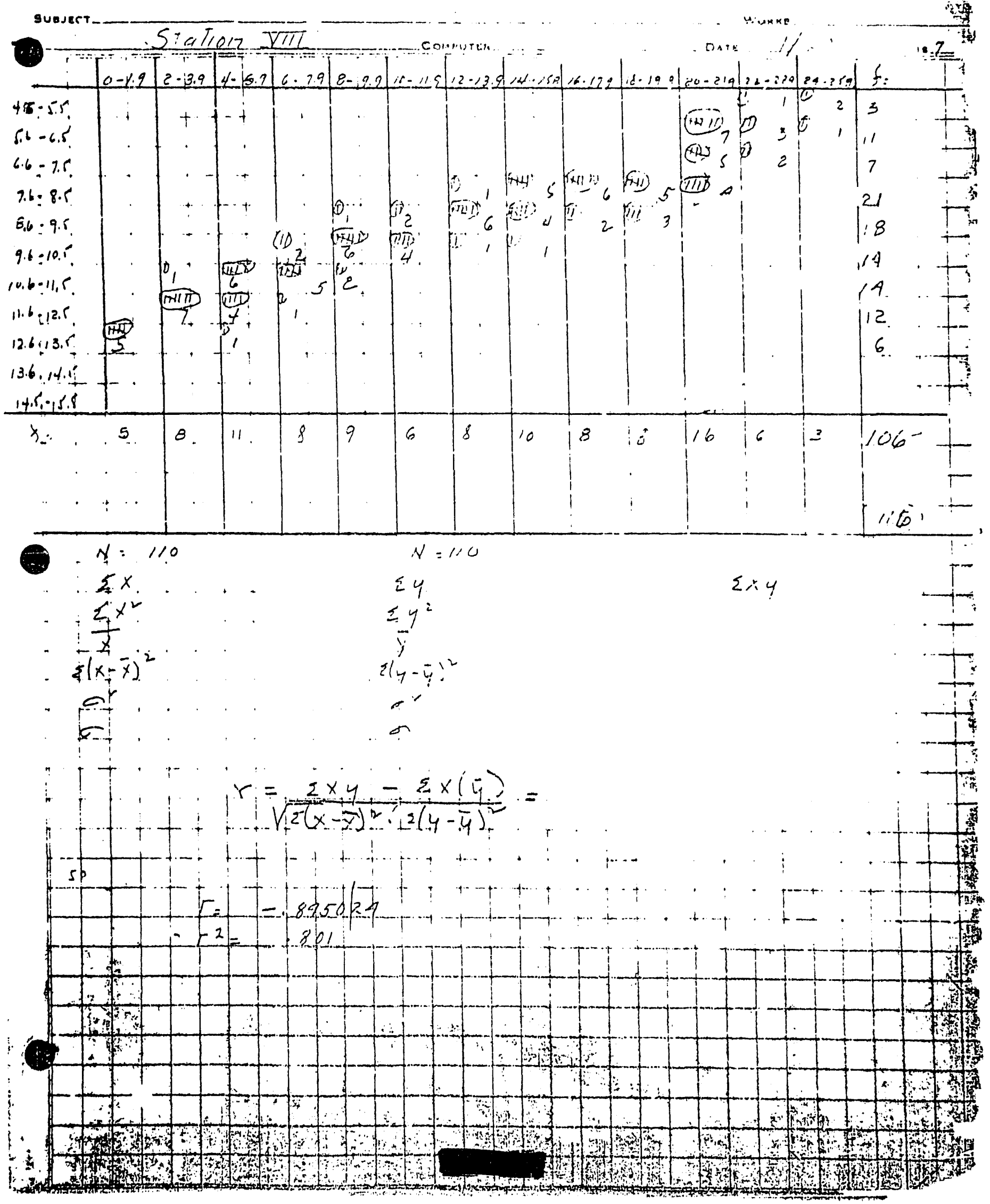




\section{DECLASSIFIED}

1 10net ton...

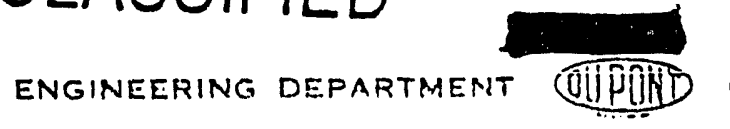

11

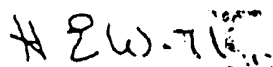

computation sheet

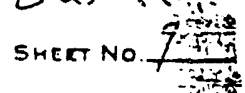

TItLE of PROS

sugject.

$T \pm 1242 \times 0-13 Q D$ $507 / 69 \sqrt{111}$

PaOs No

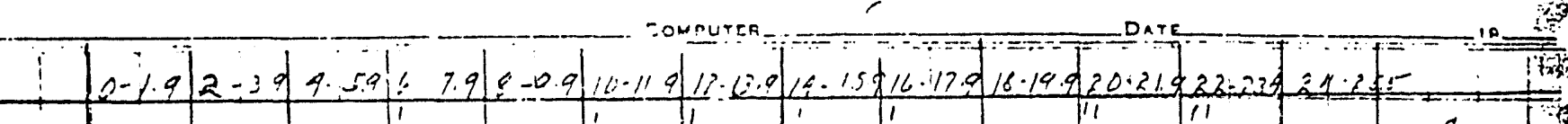

$4 \cdot .2$

$.3-.5$

$.6 \cdot .8$

$9-1.1$

1

$1.5-1.75$

$1.6-2.0$

$2.21-2 \cdot 3$

$2,9-2.6$

角7-3:9

$2.0-3.2$

5.1

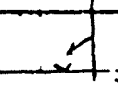

$\int^{\prime \prime}$

i

....

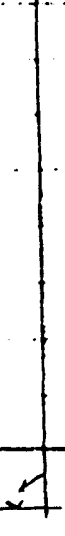

IIII j111 :!!

11 -

i. $\mid 111 \cdot 7$

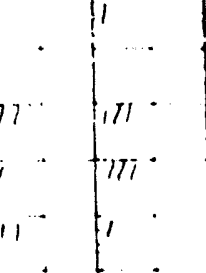

!"

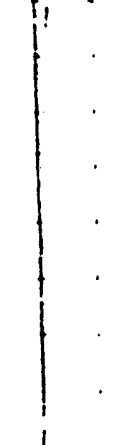

i

\begin{tabular}{l|r|r|r} 
& & & \\
\hline 11 & $?$ & $\vdots$ & \\
\hline
\end{tabular}

.

1 inc....

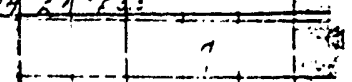

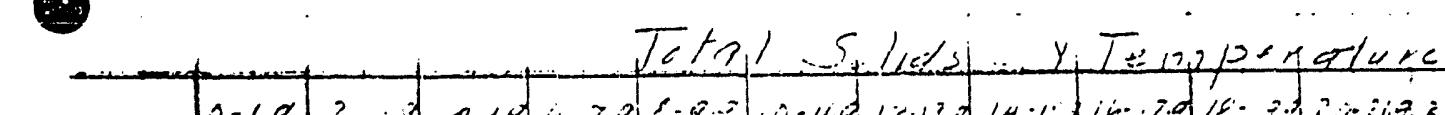

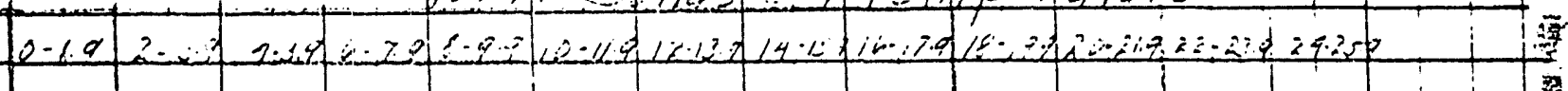

\section{10.0}

$141-130$

$12 L 140$

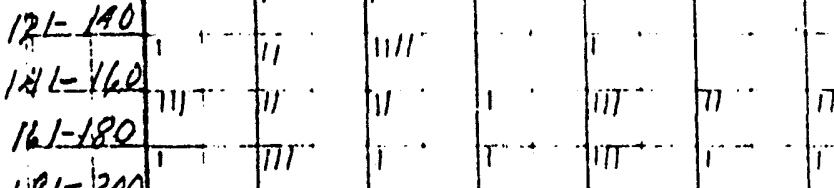

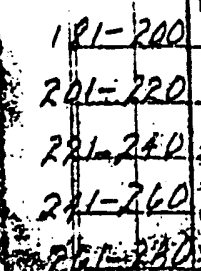

FW
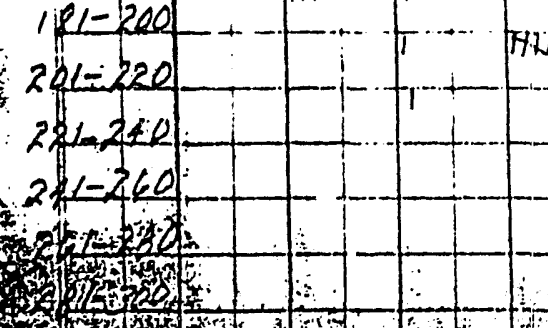

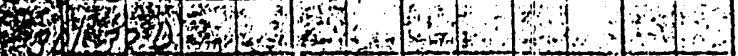

425 (3)

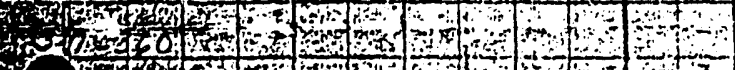

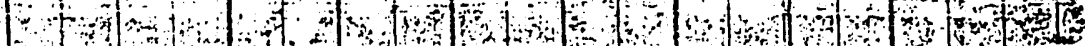

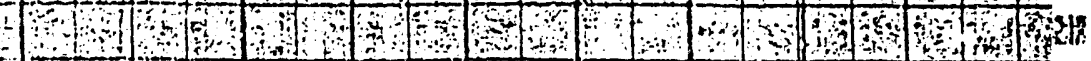

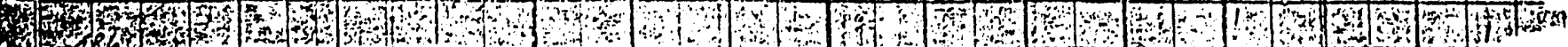

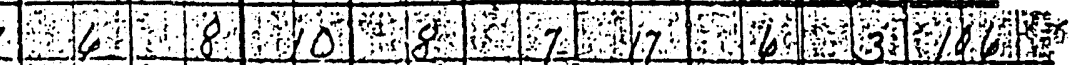

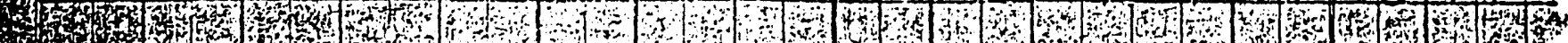

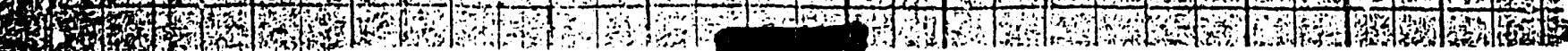


$\because . . . .$. DECLASSIFED

ENGINEERING DEPARTMENT MUPDUND COMPUTATION SHEET क $2 \omega-717$

TITLE OF PROJ

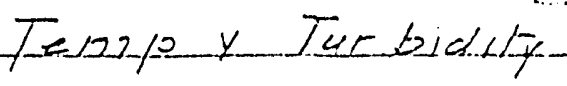

SUBuET Staliace vis WOAKs

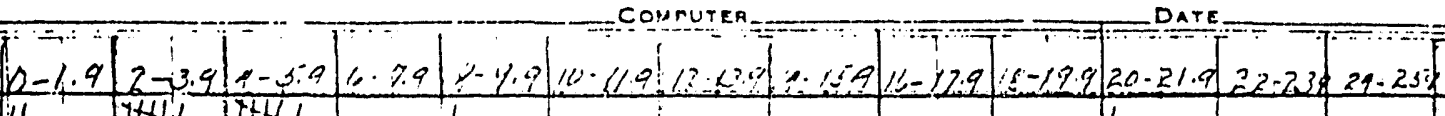

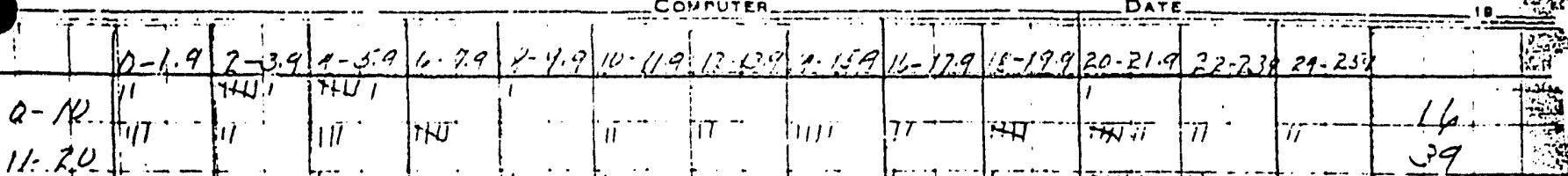
11:20.0.

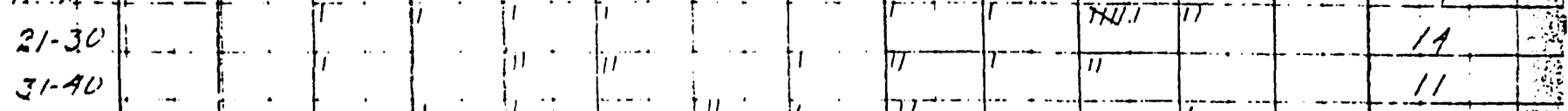
$271 \cdot 50$. $5 \% 40$ $61-20$ 81.90 -1-100

$141 \cdot 120$

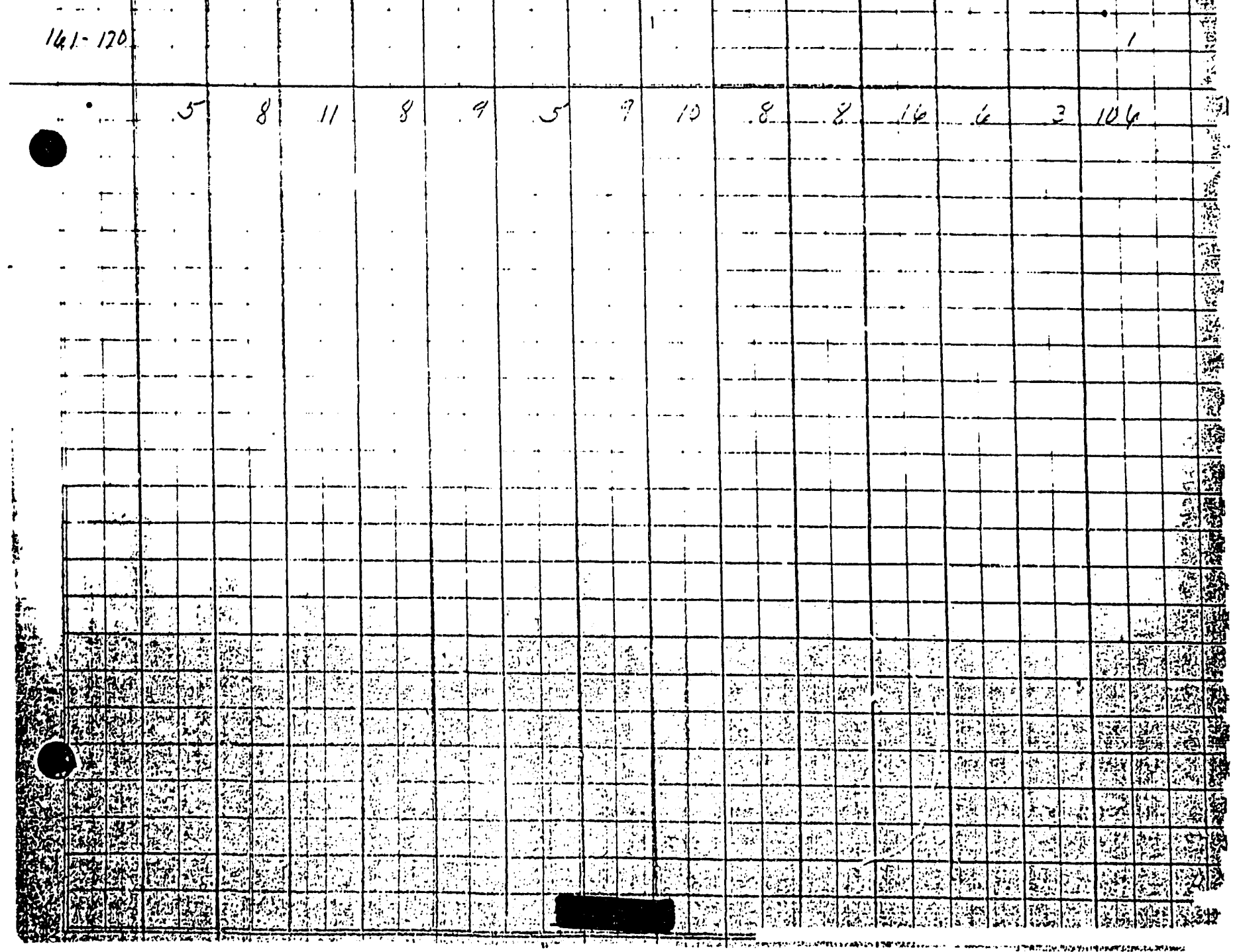




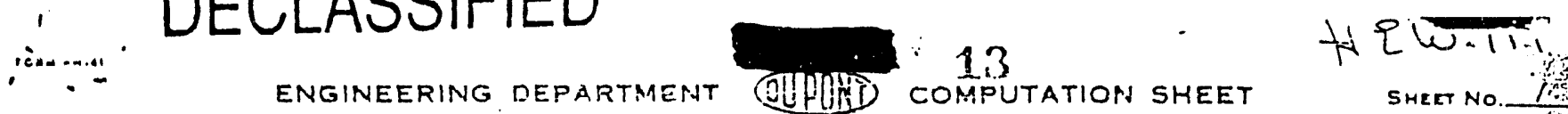

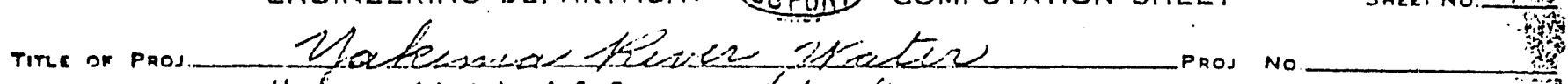

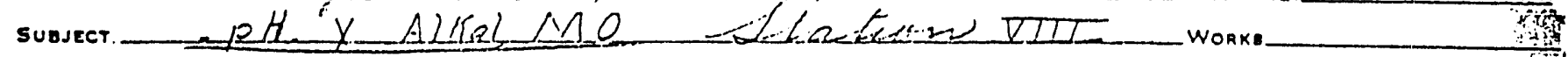

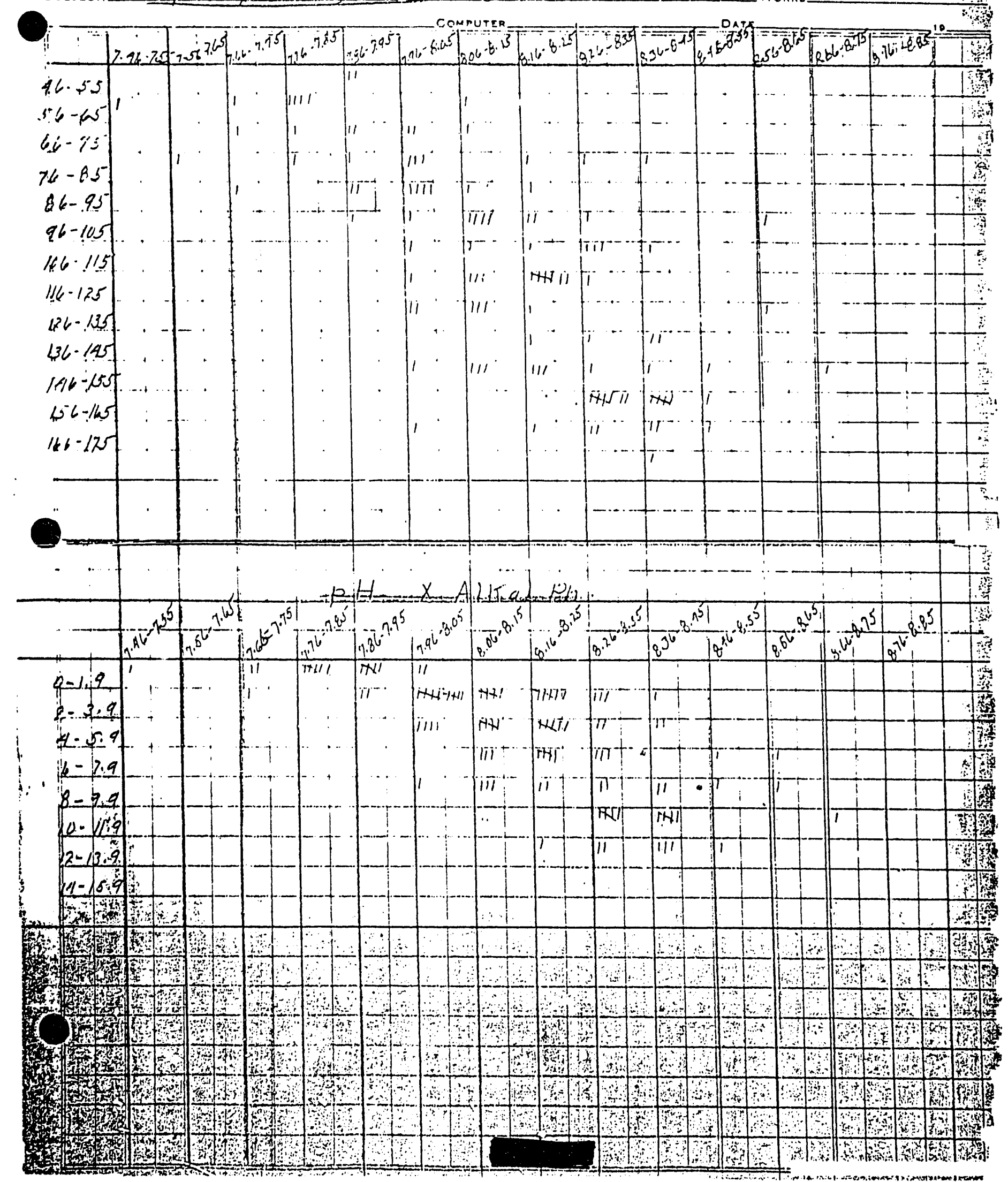




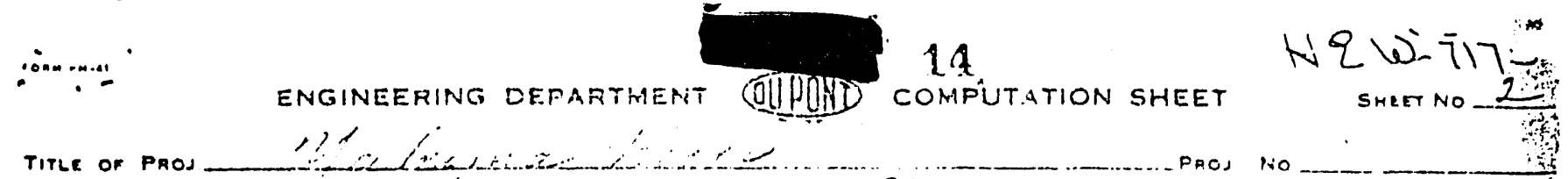
TITLe of Pros Sueser

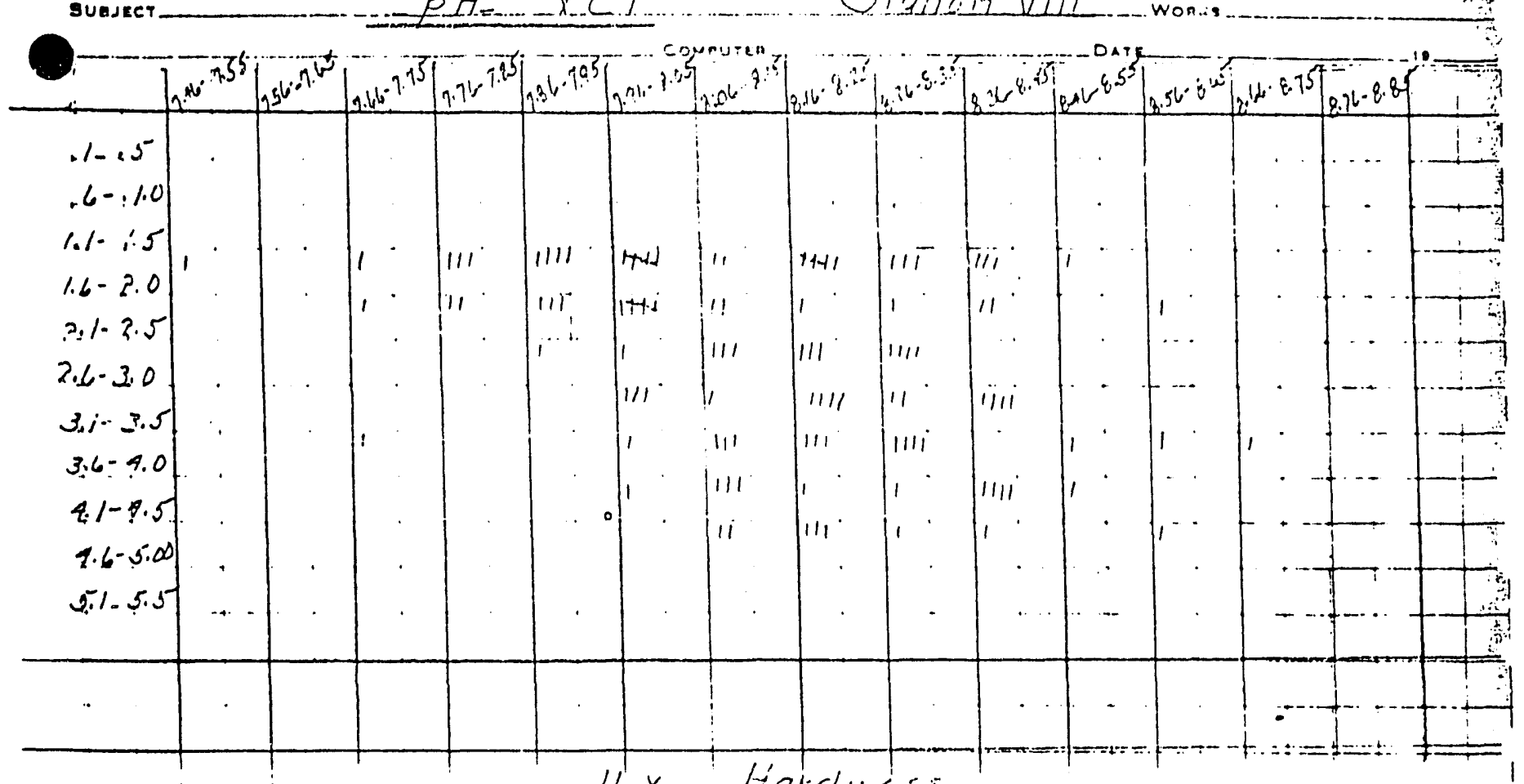

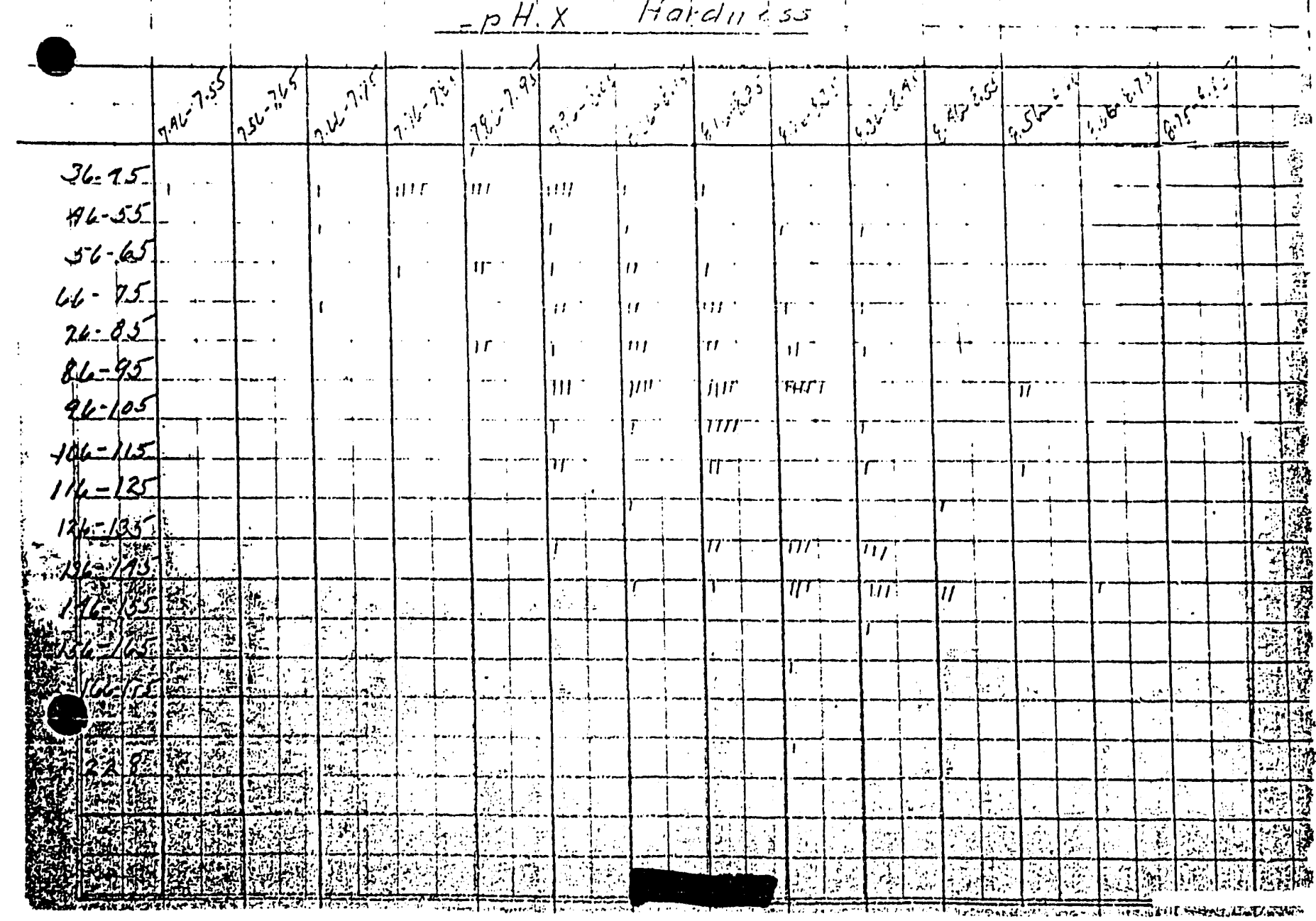




\section{DECLASSIFIED}

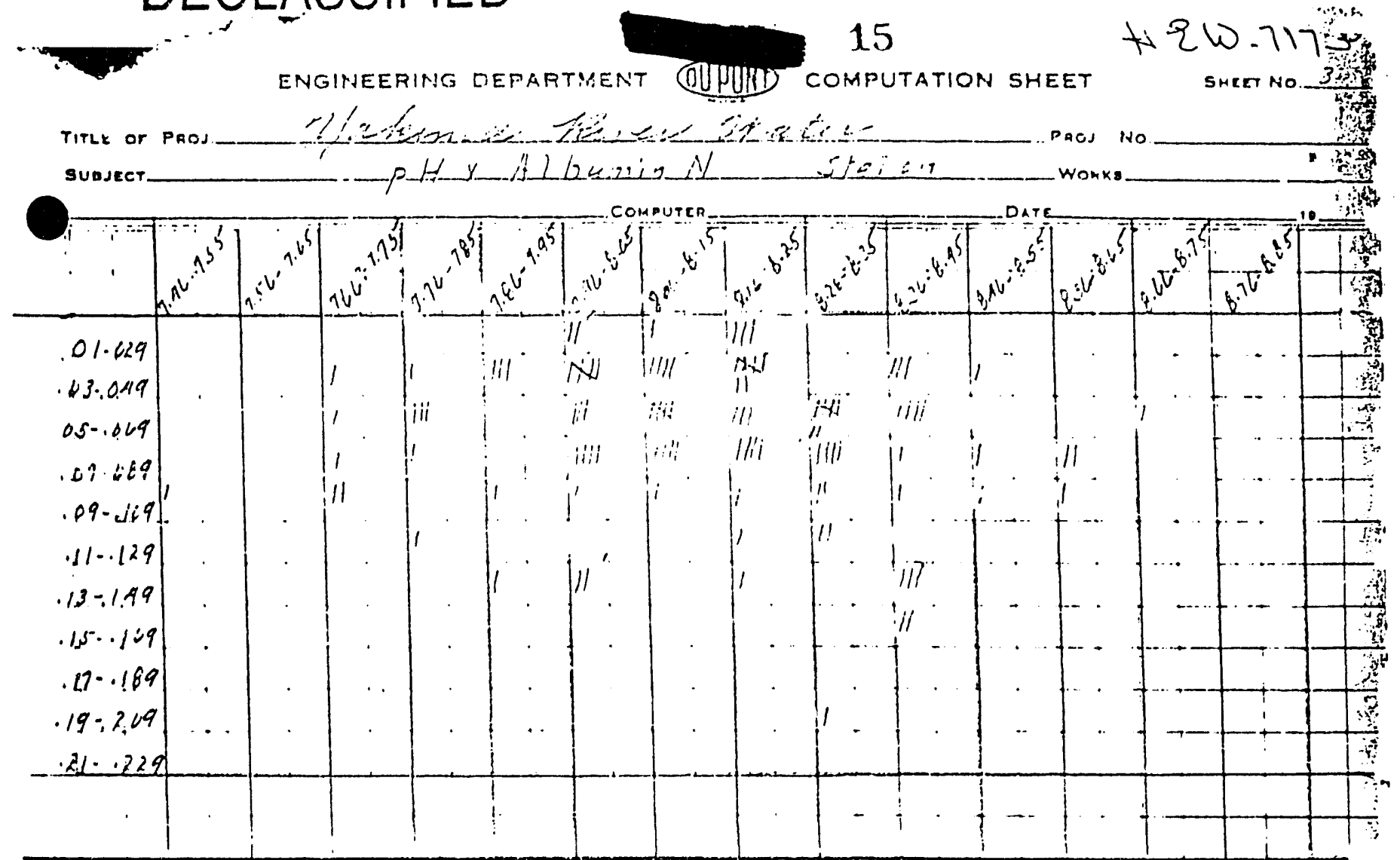

$-12 H: X . M H_{3}$

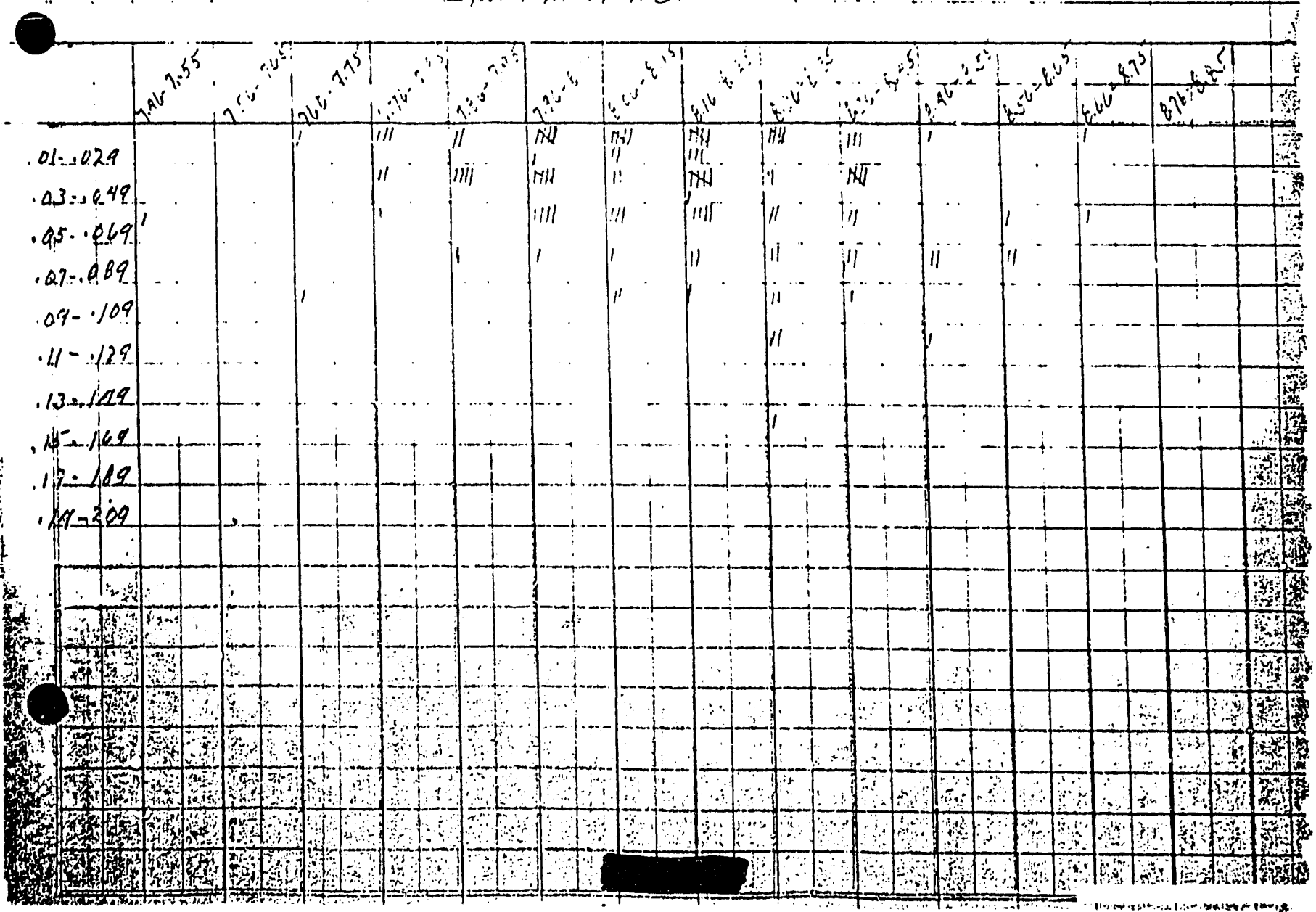

DECLASSIFIED 
DECLASSIFIED

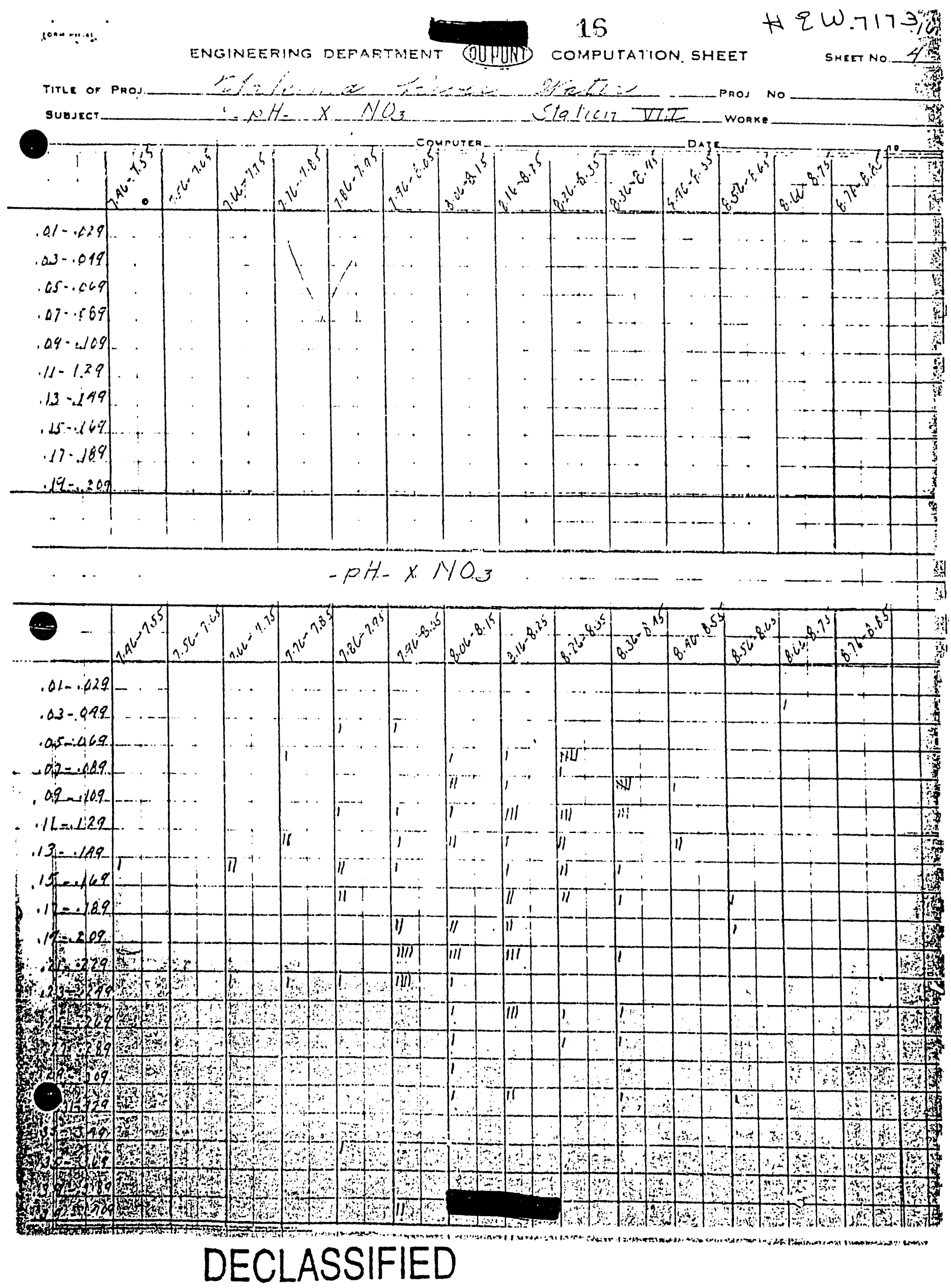




\section{DECLASSIFIED}

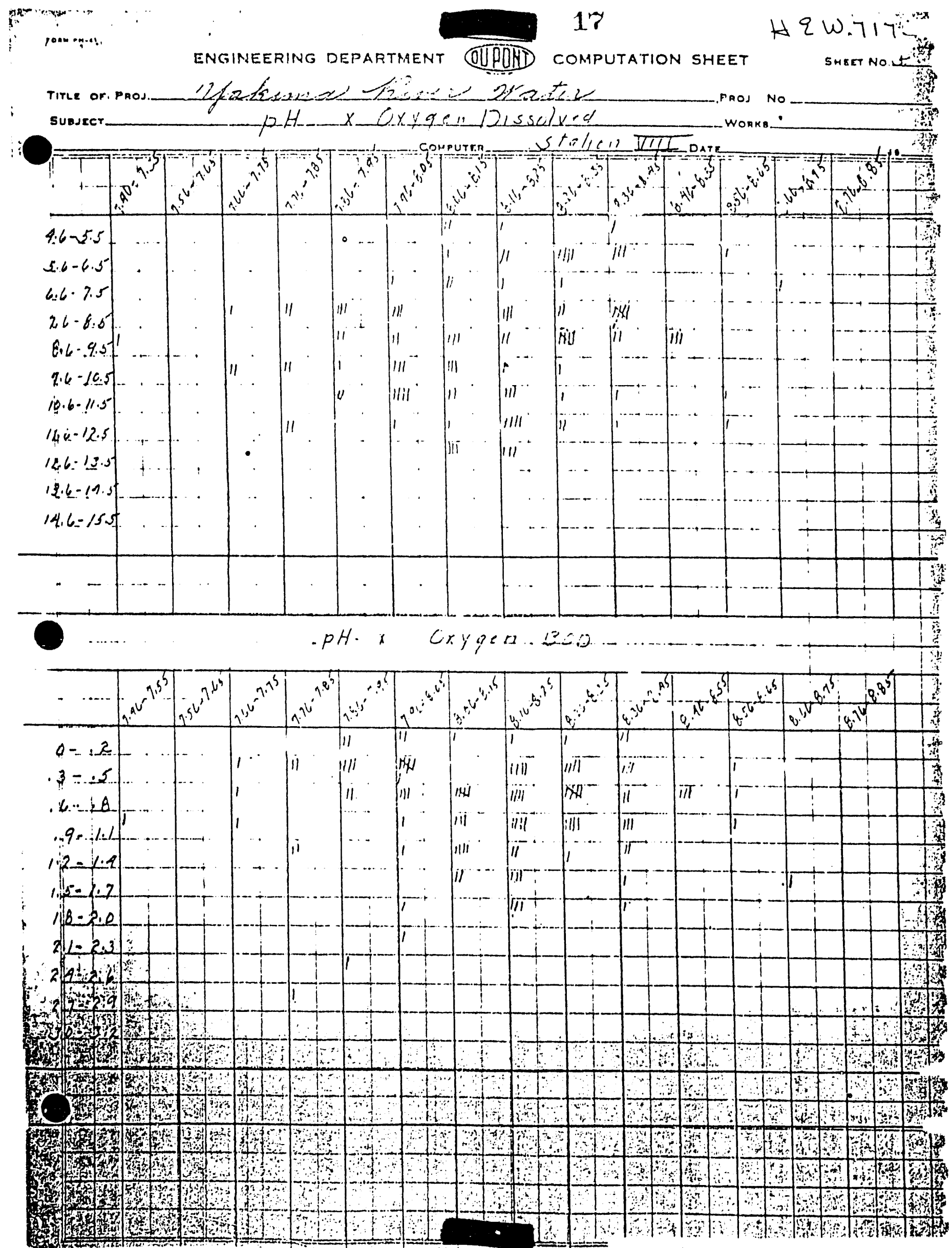




\section{DECLASSIFIED}

$+1.46$

ENGINEERITIG DEFARTMENT OUPOND COMHBTATION SHEET H $2 \omega-717$ SHERT NO ne or PROS. 1.7 re St:1 $1: 17$ पूSit:- work $t^{2}$

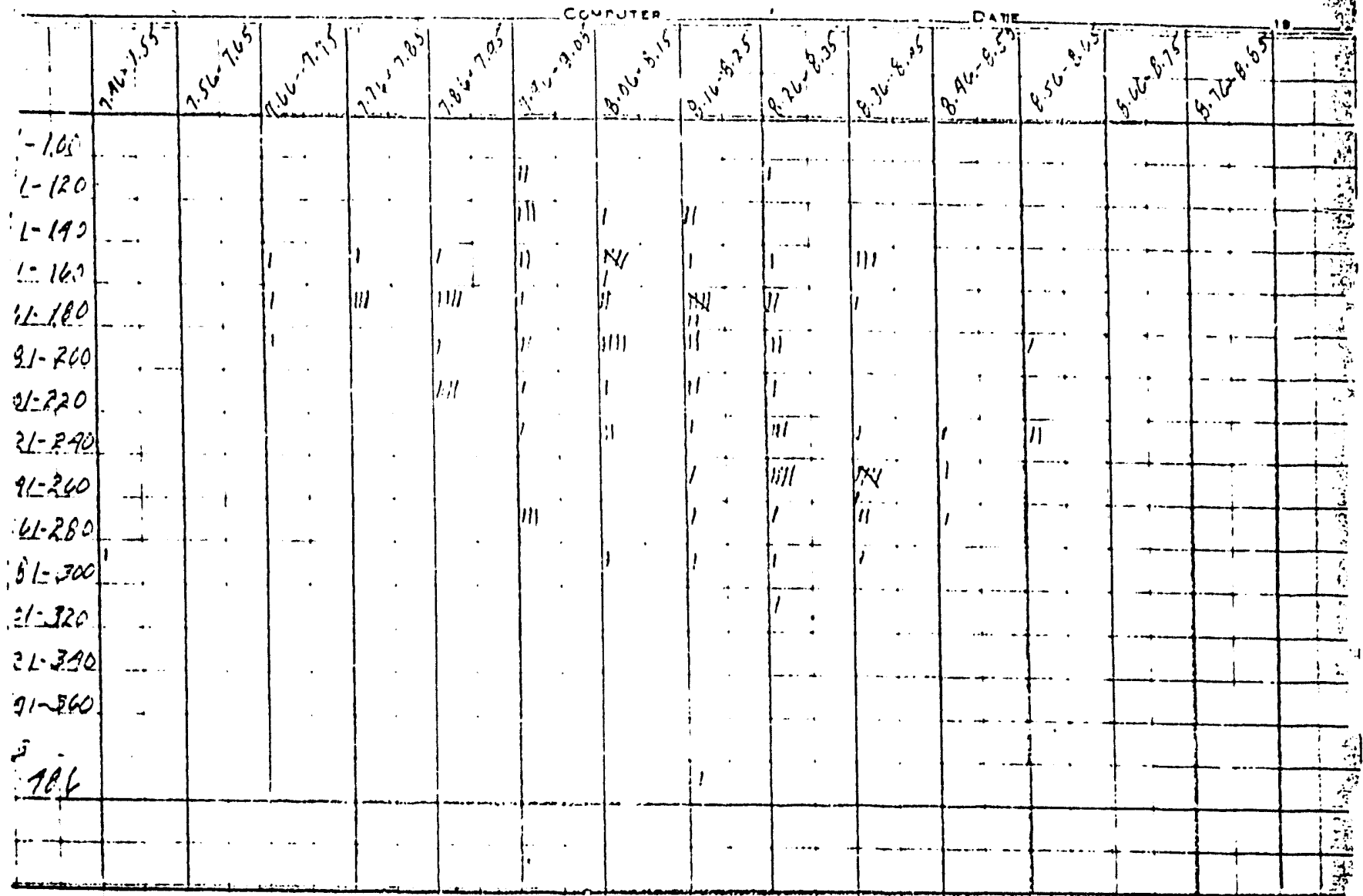

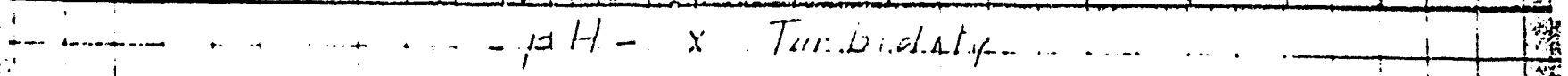

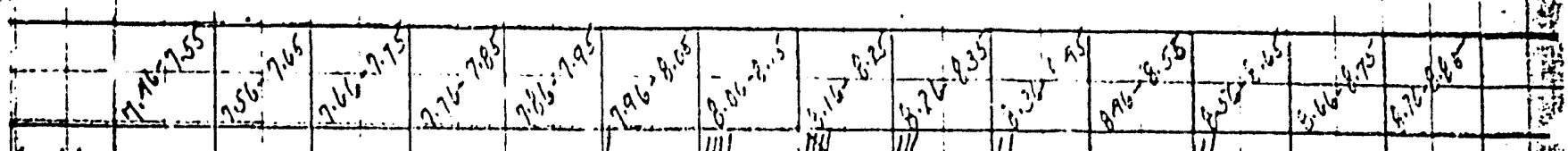

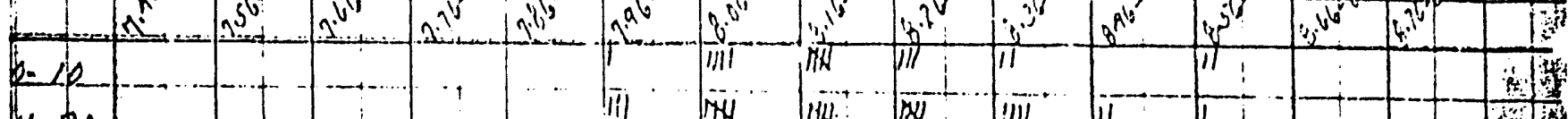
$1-20$ Bis 30 


\section{DECLASSIFIED}

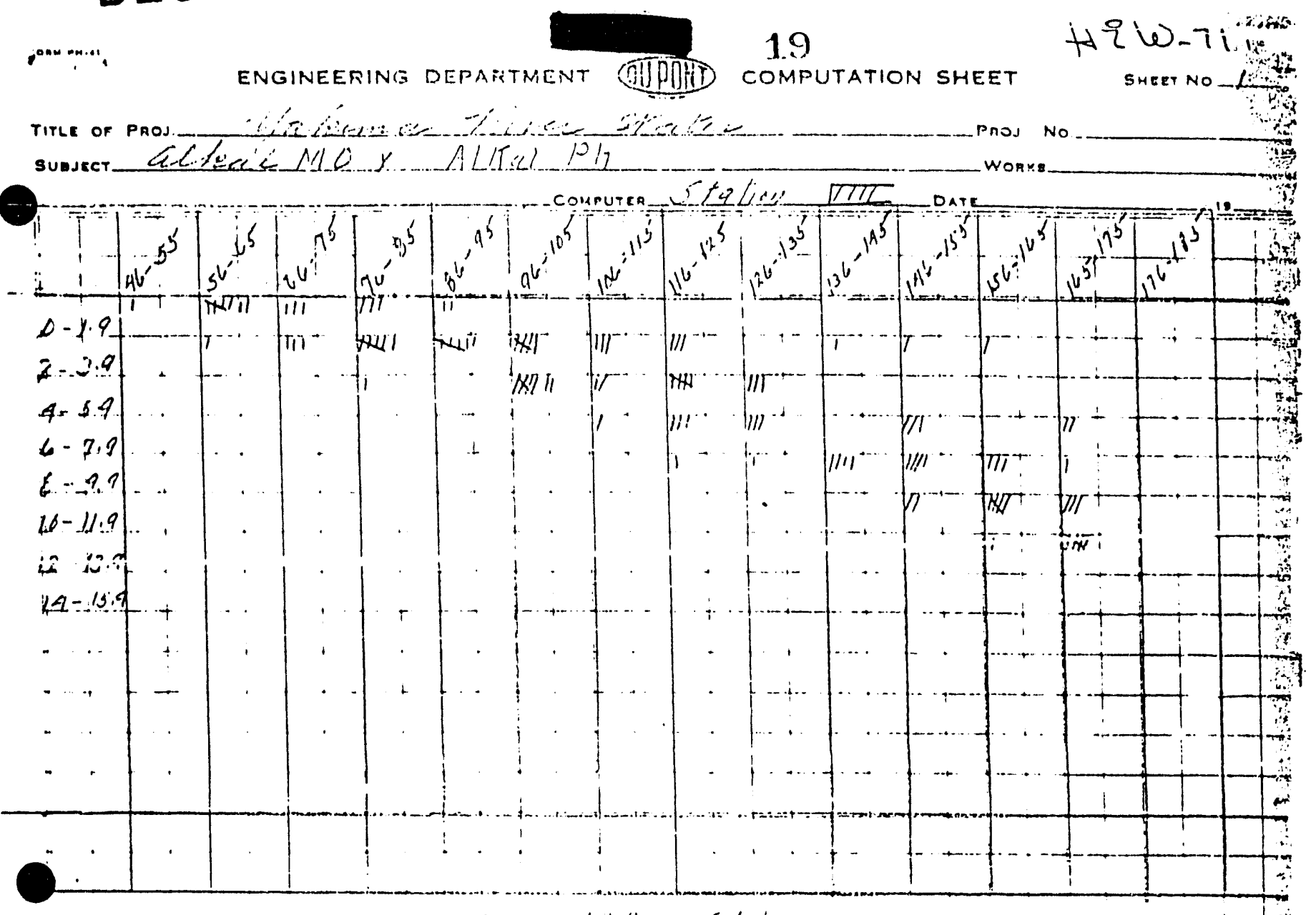

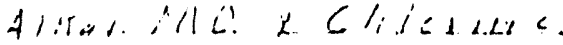

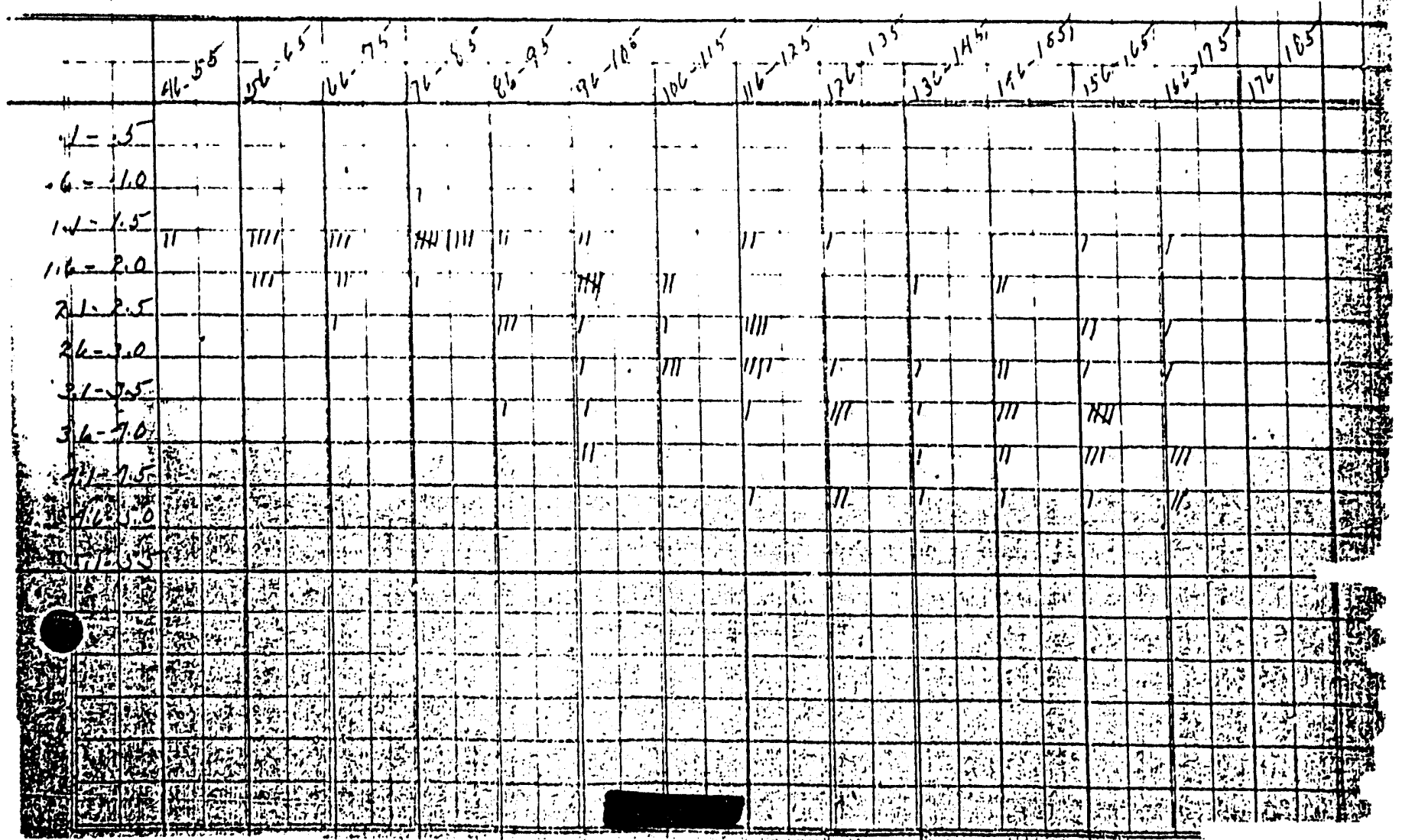


DECLASSIFIED
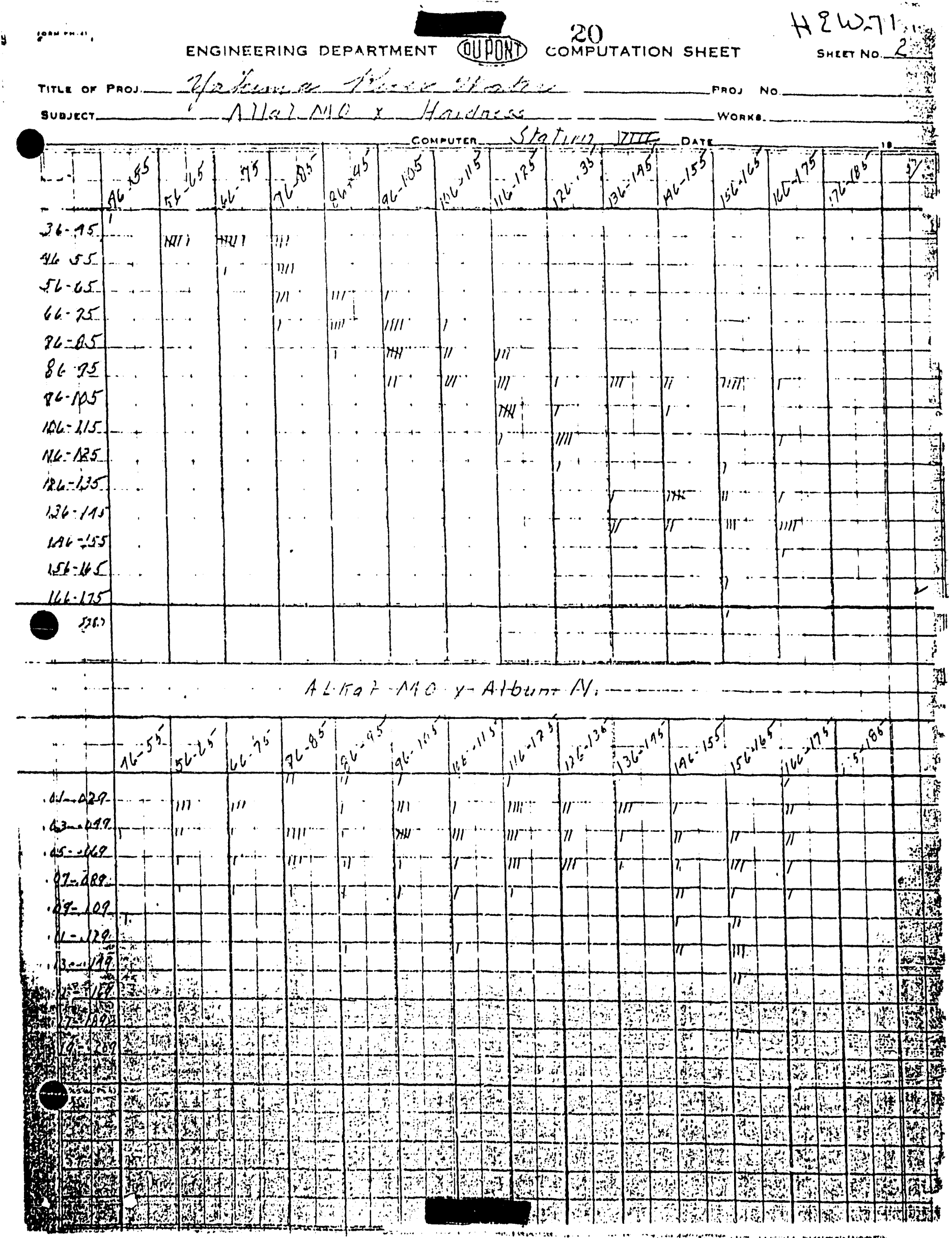

\section{DECLASSIFIED}




\section{DECLASSIFIED}

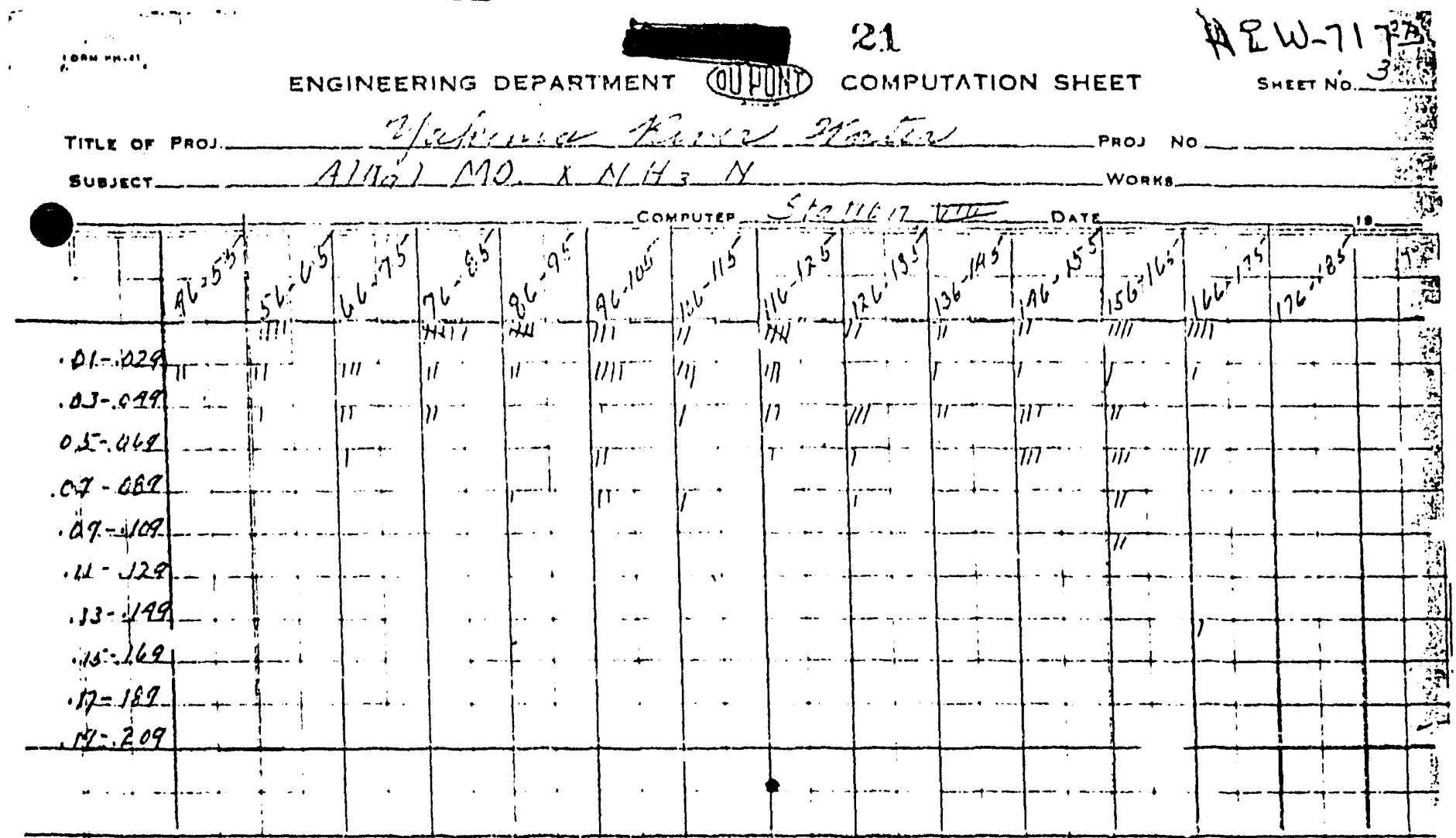

- Allios Mela.x Marn

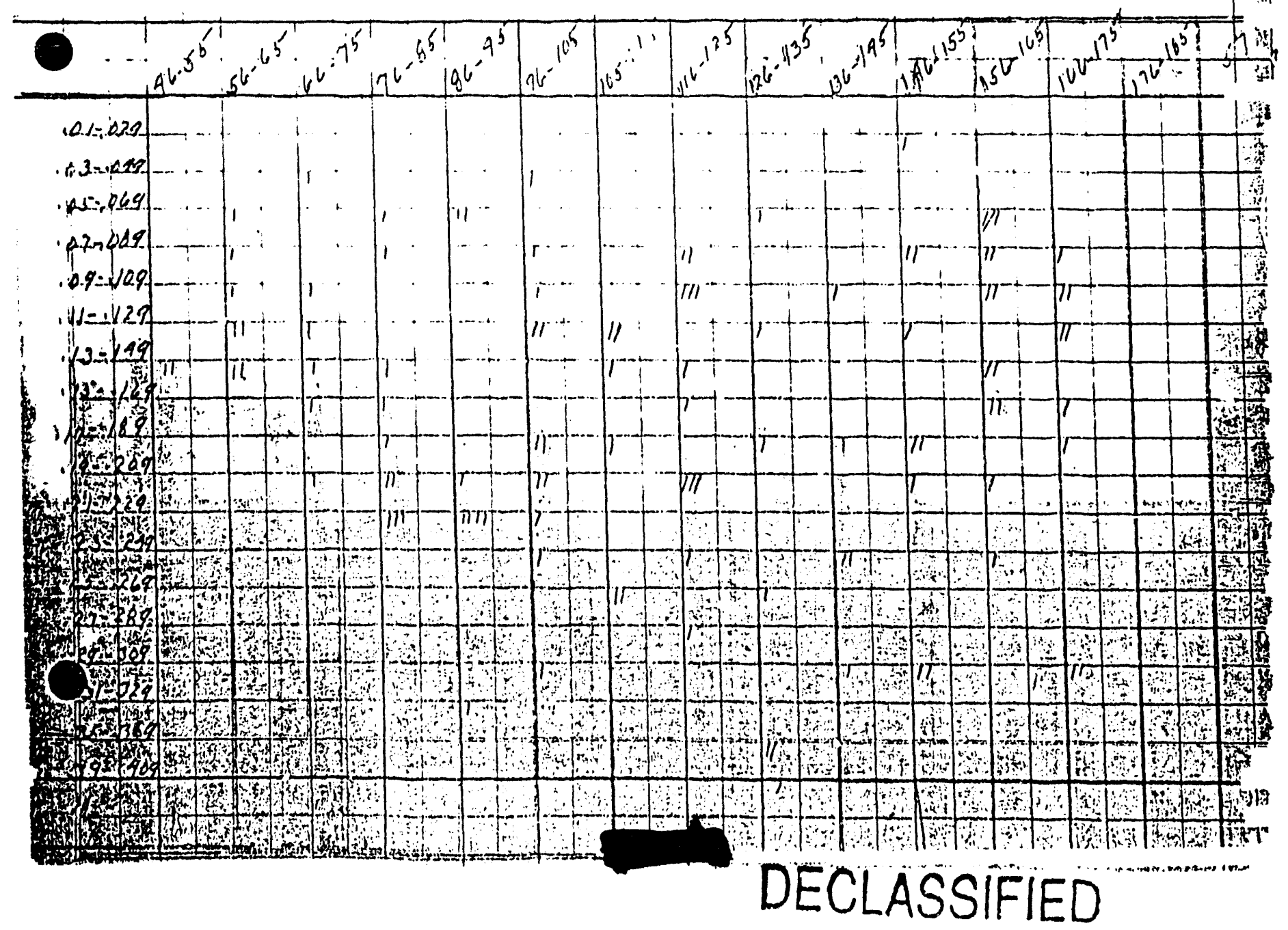


DECLASSIFIED

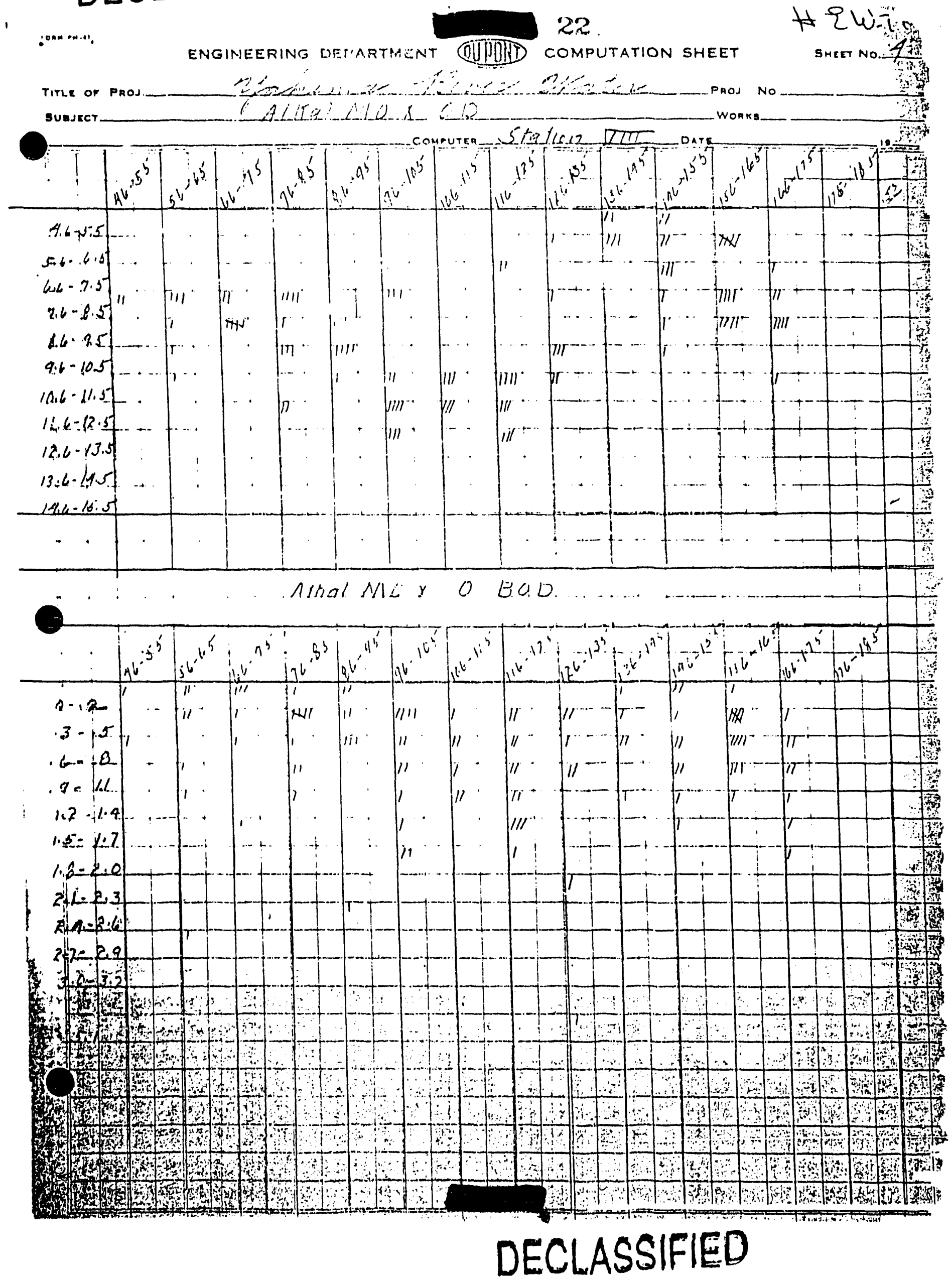




\section{DECLASSIFIED}

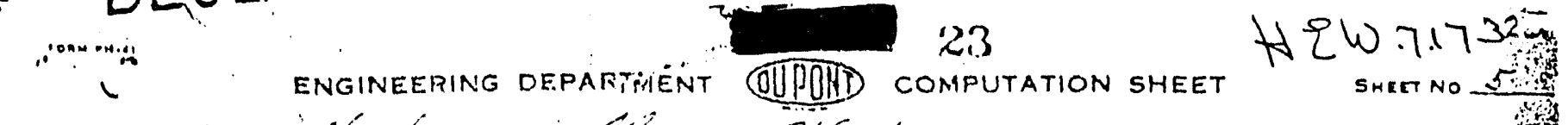

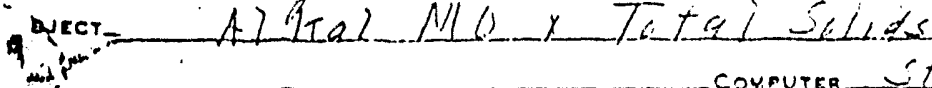

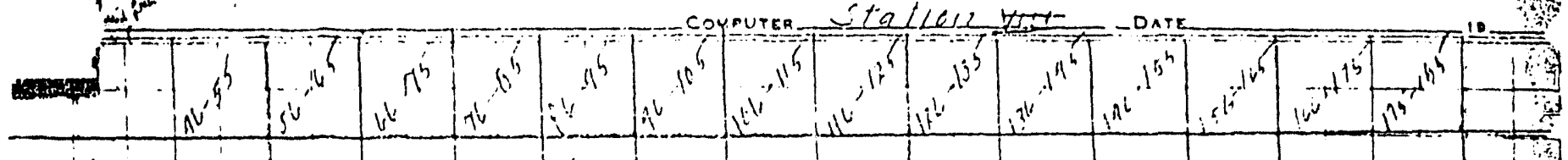

\section{$81=100$}

101:120.

121. 190

$141+160$

$161-480$

$1.81: 200$

$201 .-220$

$221: 240$

$2 \pi 1-260$

$201-280$

$201-300$

$301: 320$

$321 .=310$

$341-360$

I.

486

0.13

1

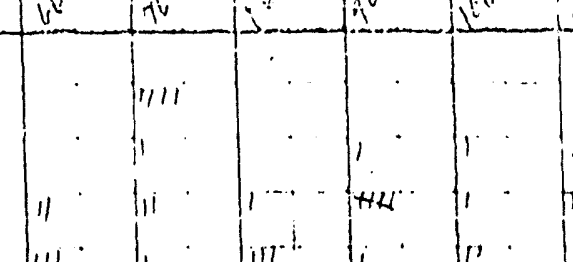




\section{DECLASSIFIED}

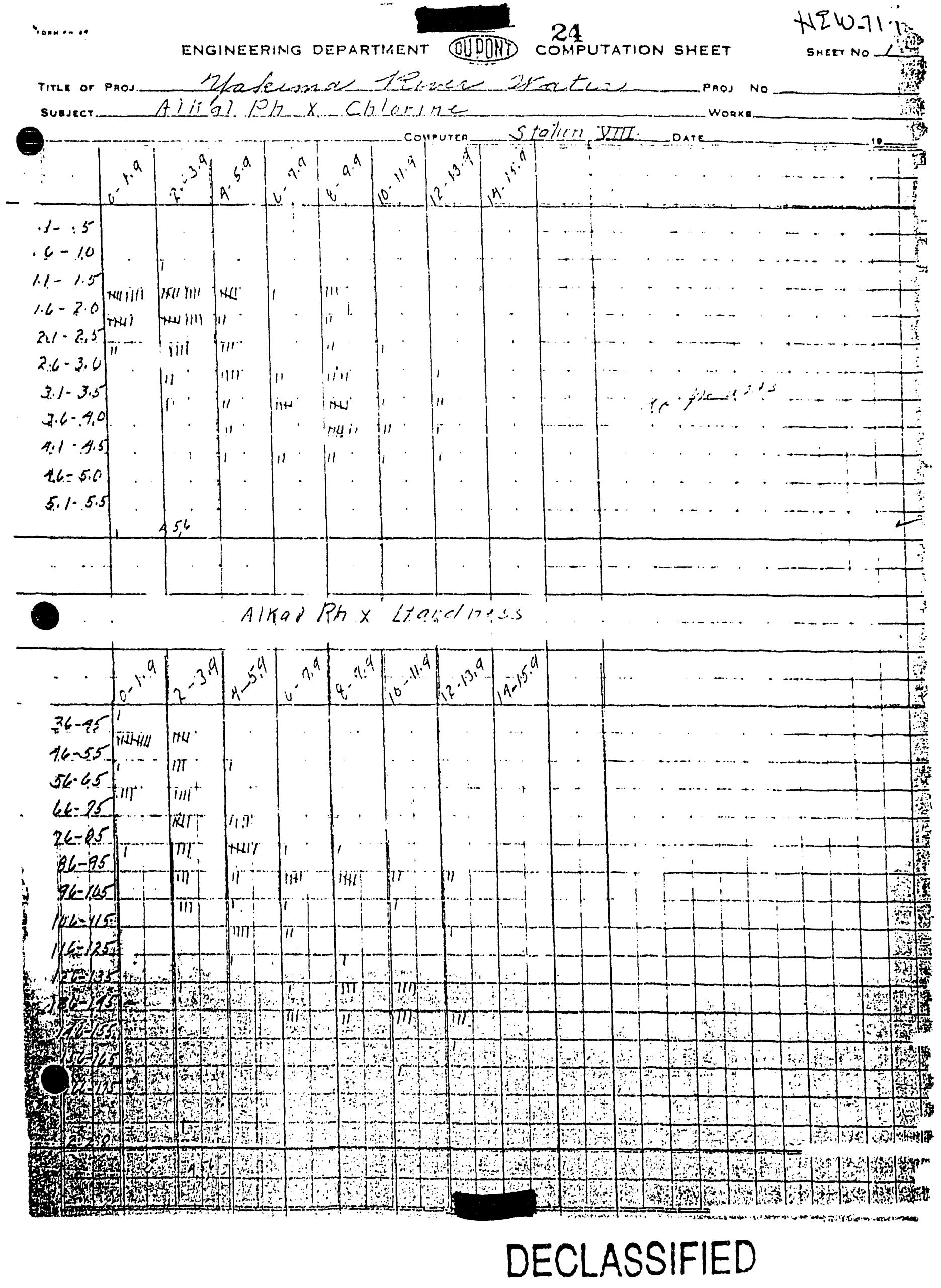


DECLASSIFIED

k ion m.a."

ENGINEERING DEPARTMENT OUPOND COMPUTATION SHEET.

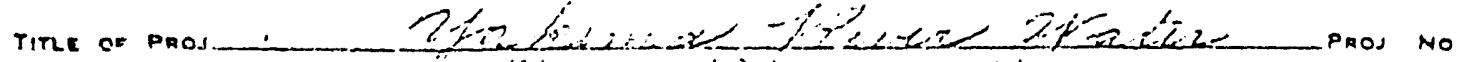

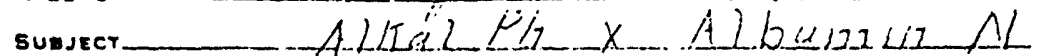
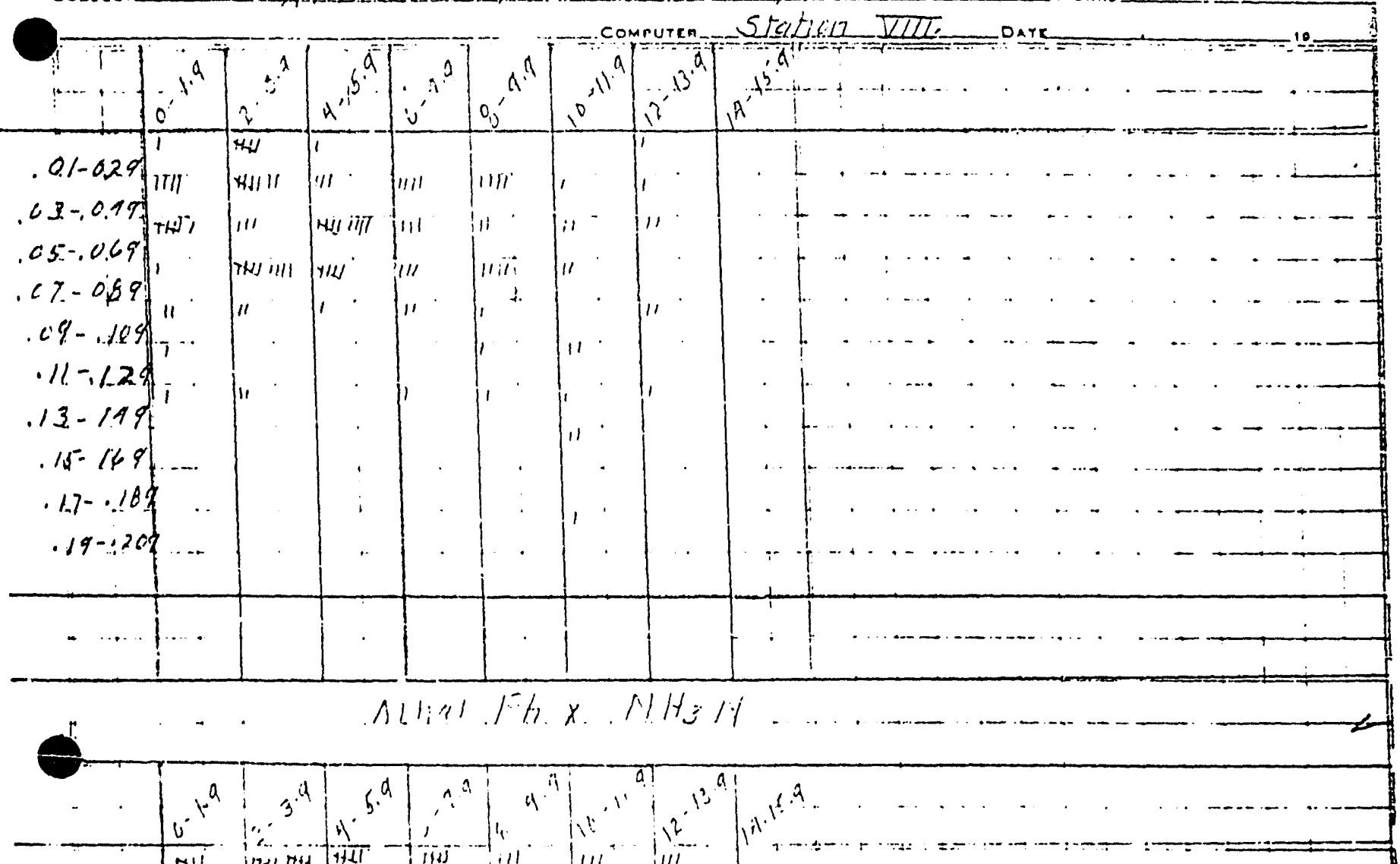

$.01-0.4$

$.3-0419$

$.05: 069$

$.27 \cdot 489$

$.09-1.09$

. $11=1<29$

.13-.219. ....

- 15.

$.72=0.189$

- 90209

nis
nist 


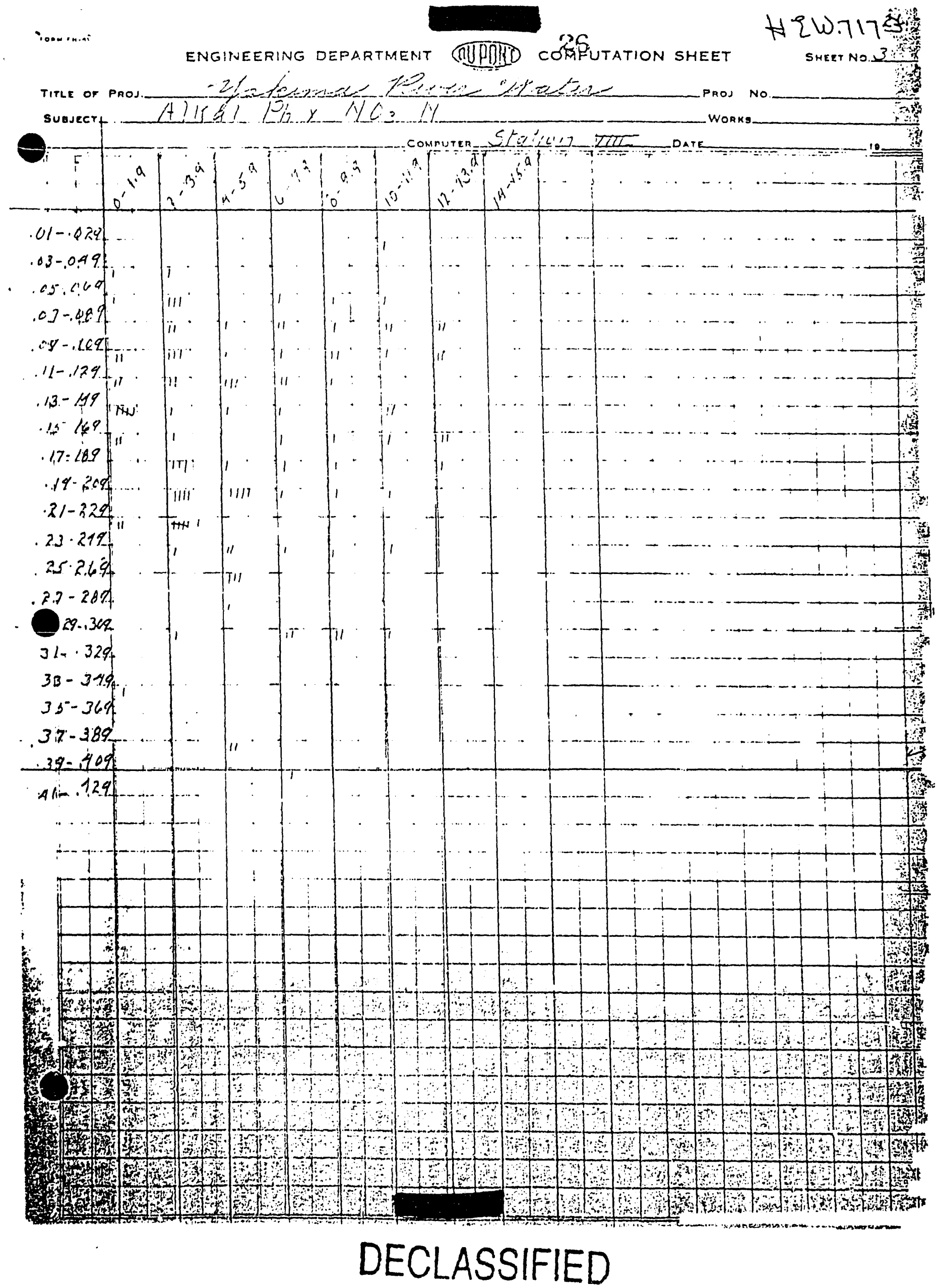




\section{DECLASSIFIED}

Vone mos

ENGINEERING DEPARTMENT (OUPIIIS COMPUTATION SHEET

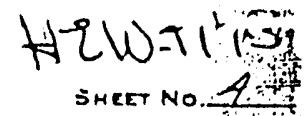
TITLE of Poos. To No

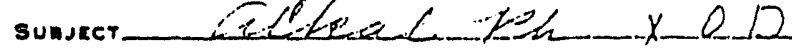
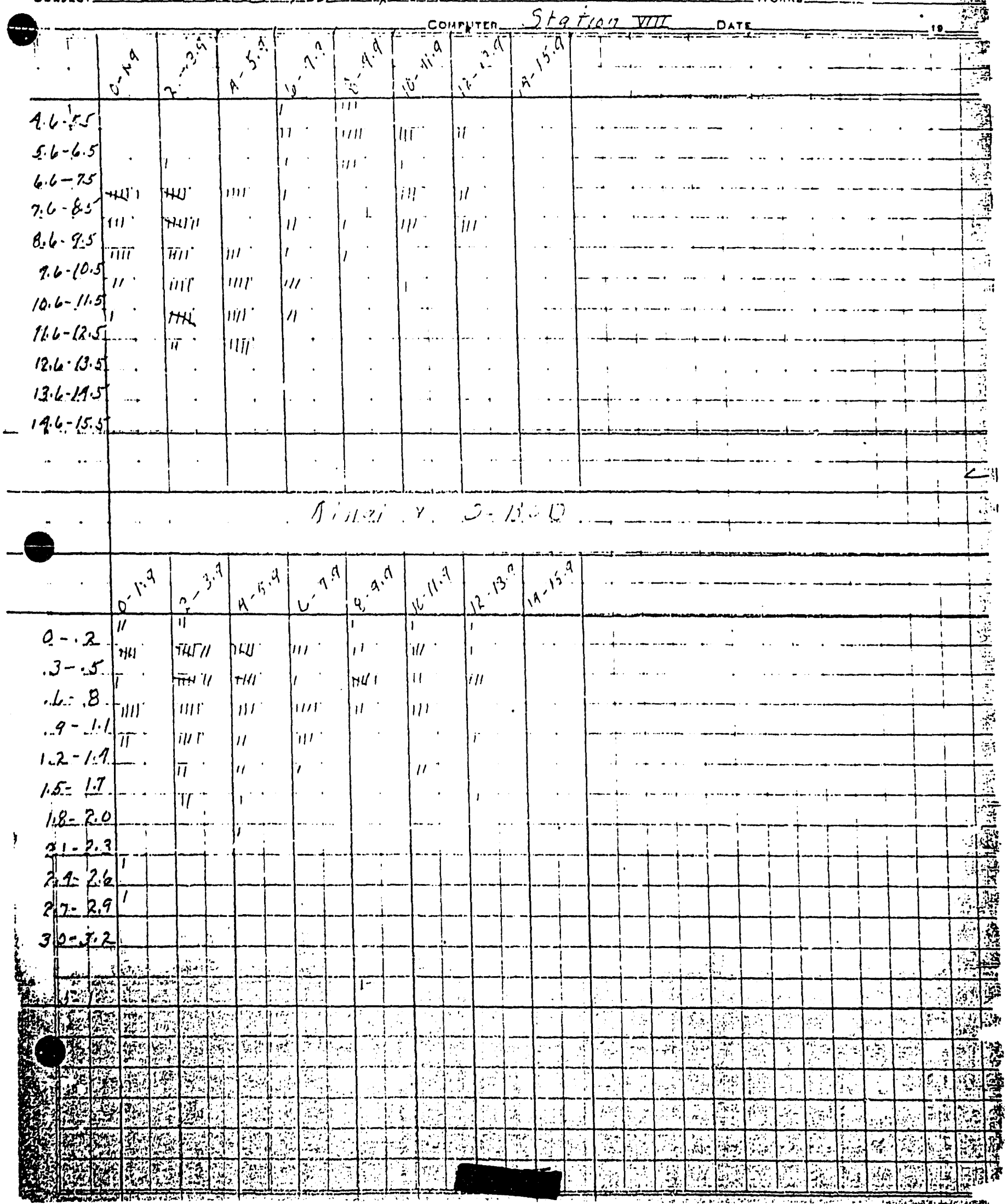


\section{$\therefore$ DECLASSIFIED $28 \quad+2 \omega-7173$ \\ ENGINEERING DERARTMENT (OUPOID COMPUTATION SHEET \\ SHEET NO.j}

TITL or PROS_

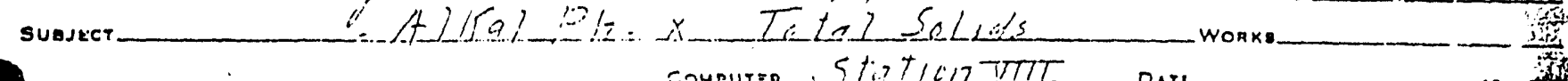
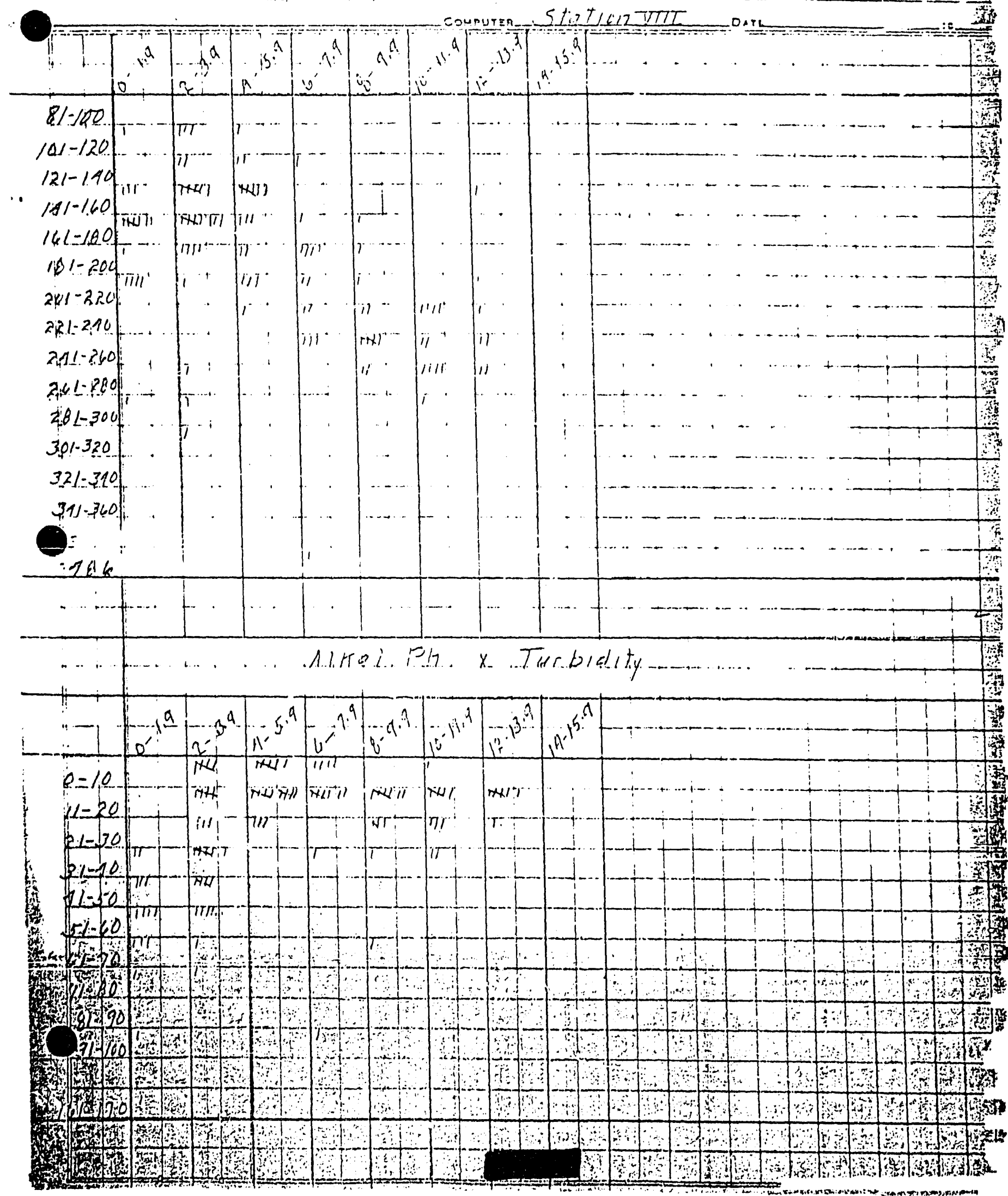

DECLASSIFIED 


\section{DECLASŚFFIIED}

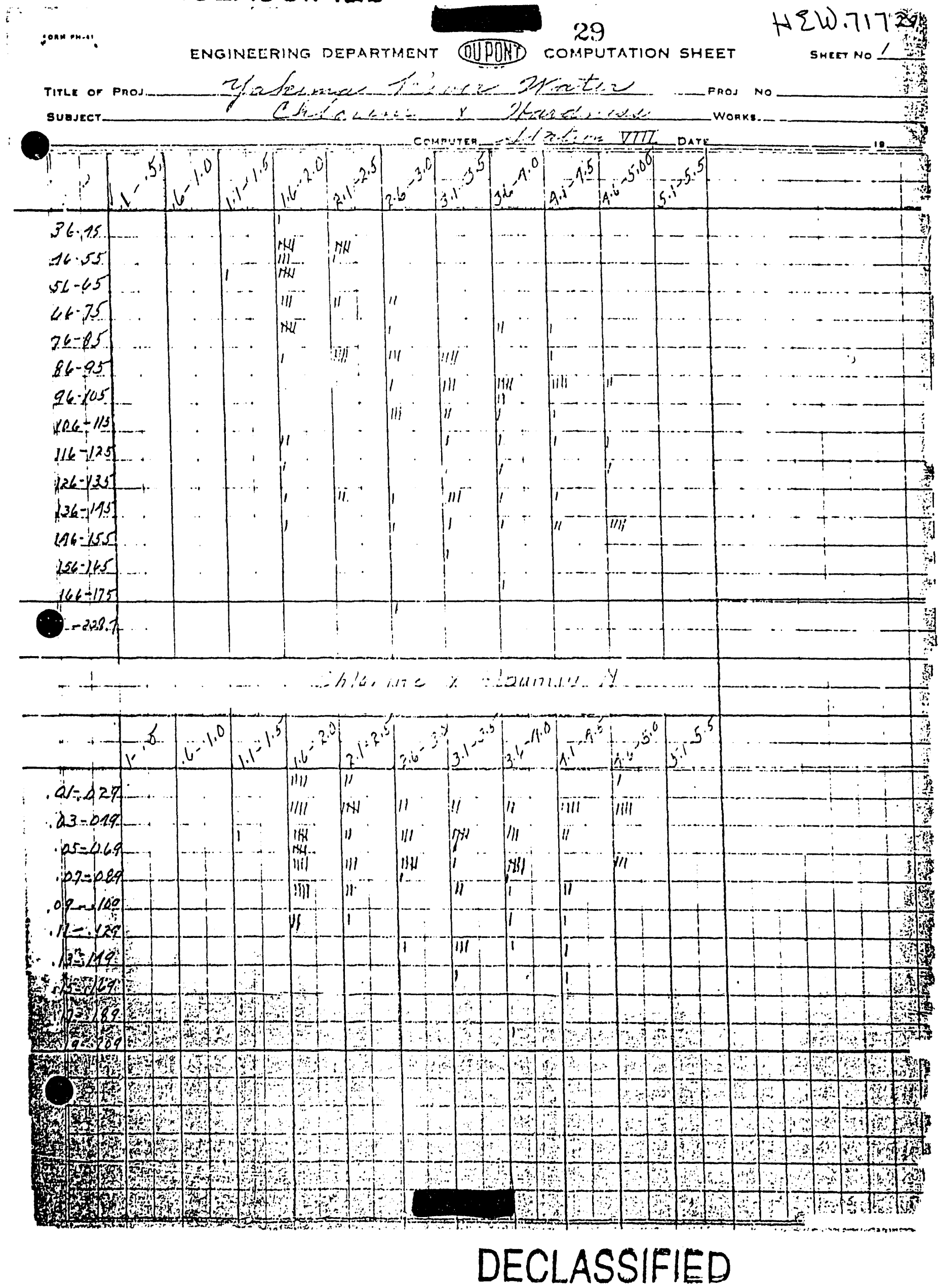




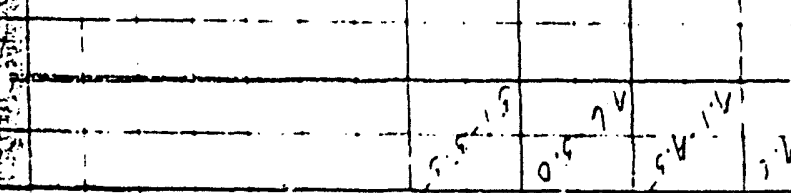
$\div 111 \div \square$

\section{$-1$} II 


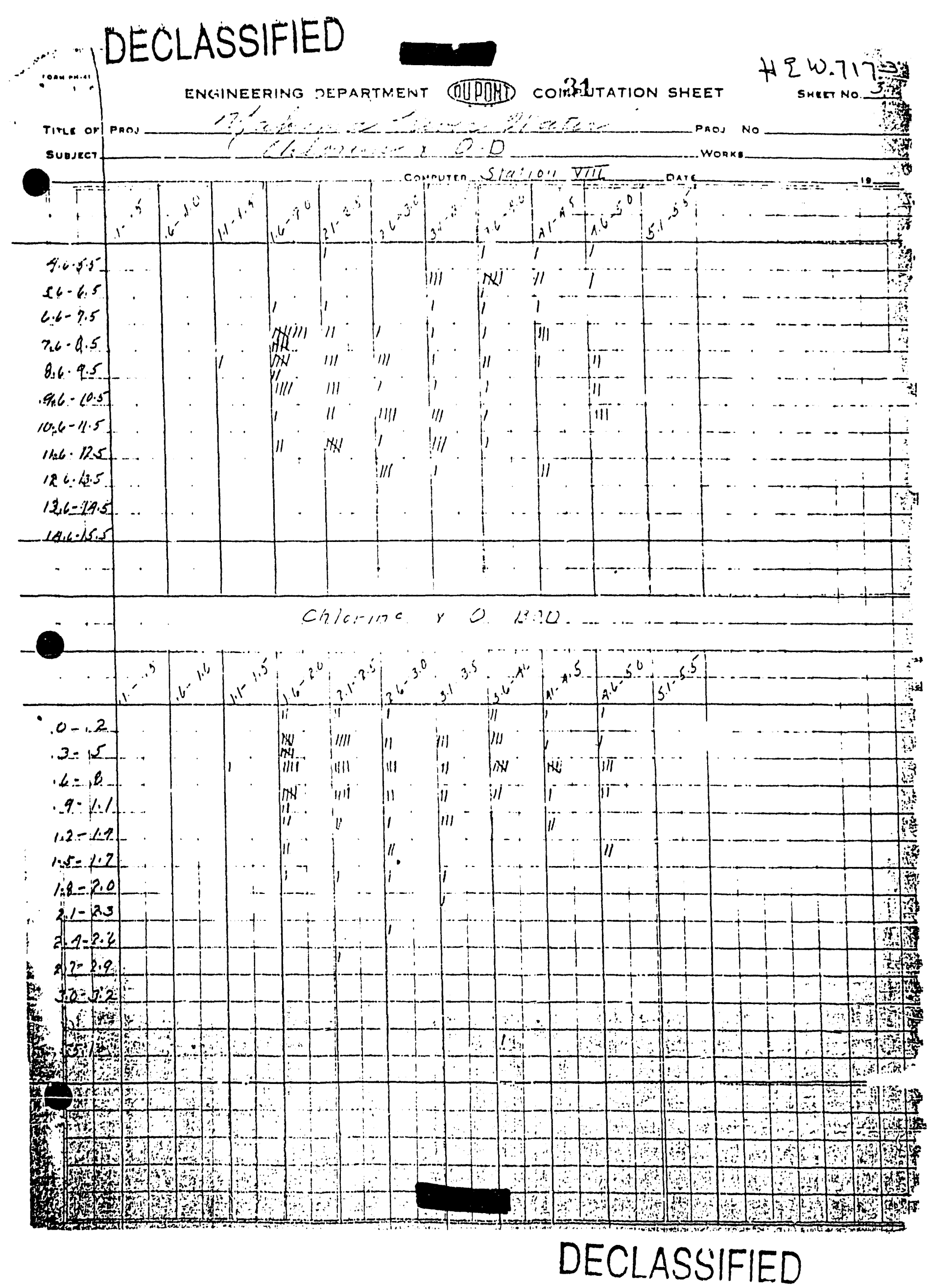


....... 32

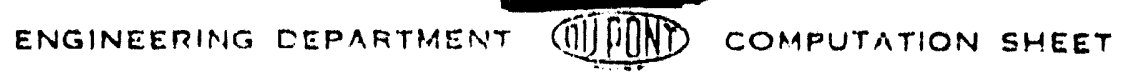

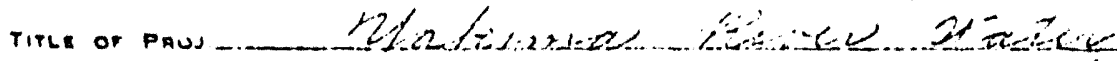

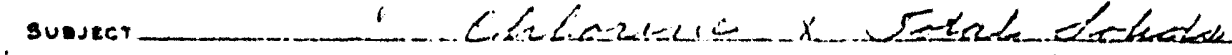

is

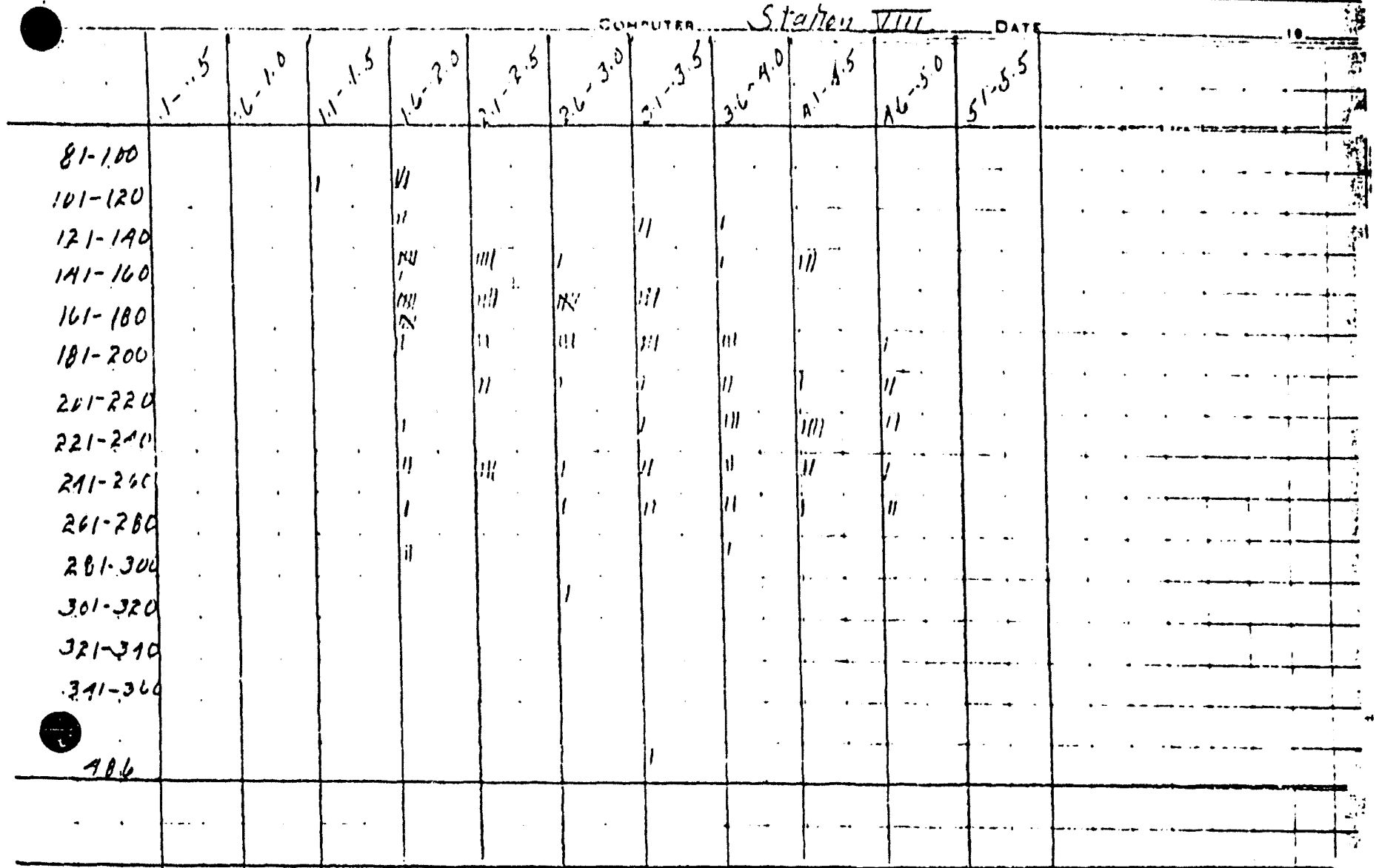

Chlorine $x$ Testbidily

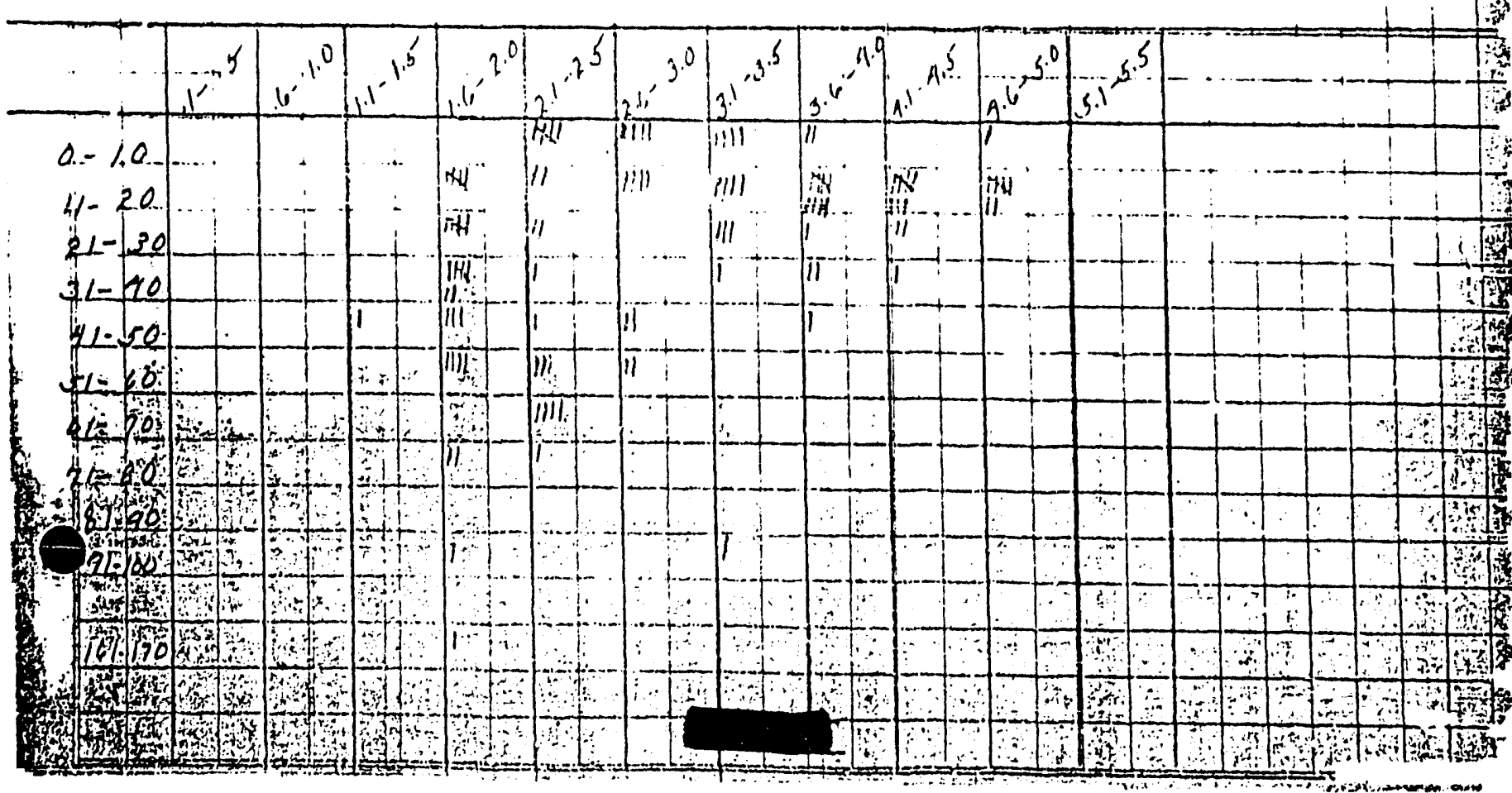




\section{DECLASSIFIED}

$\because \%$

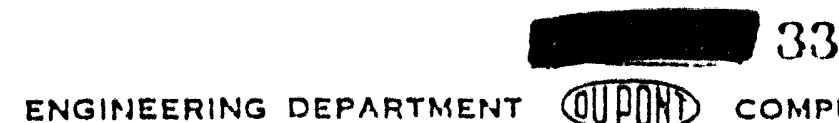

TitLE of PMos. ENGINEERING DEPARTMENT OUPOPD COMPUTATION SHEET $H \sum W 71$

BUDder. $2 t a r a t 2 a x$

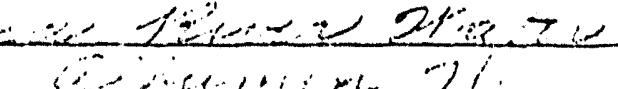
wonxo

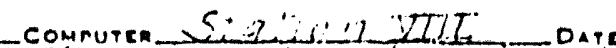

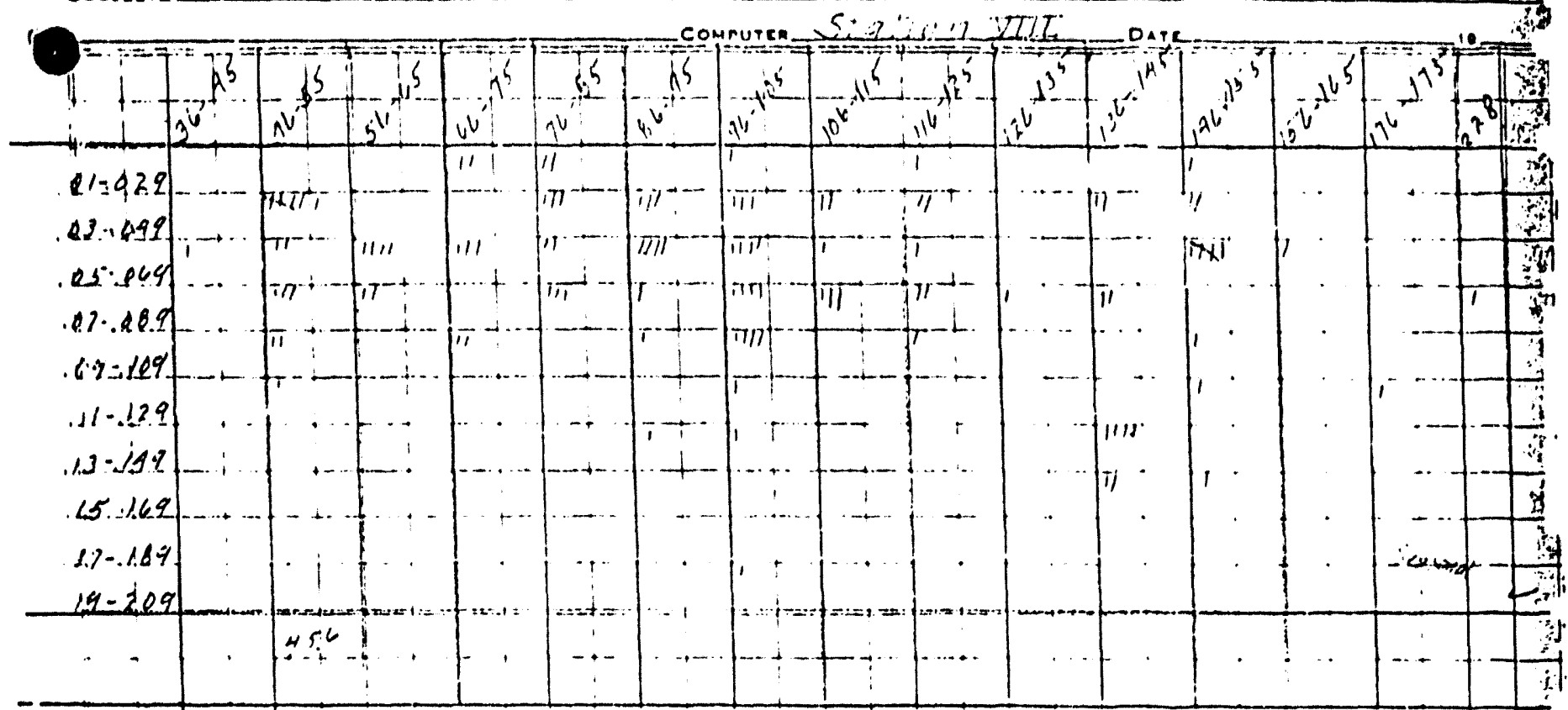

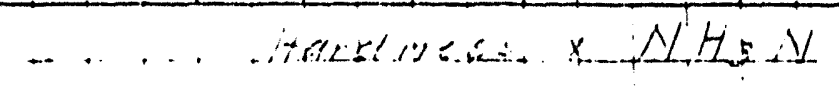

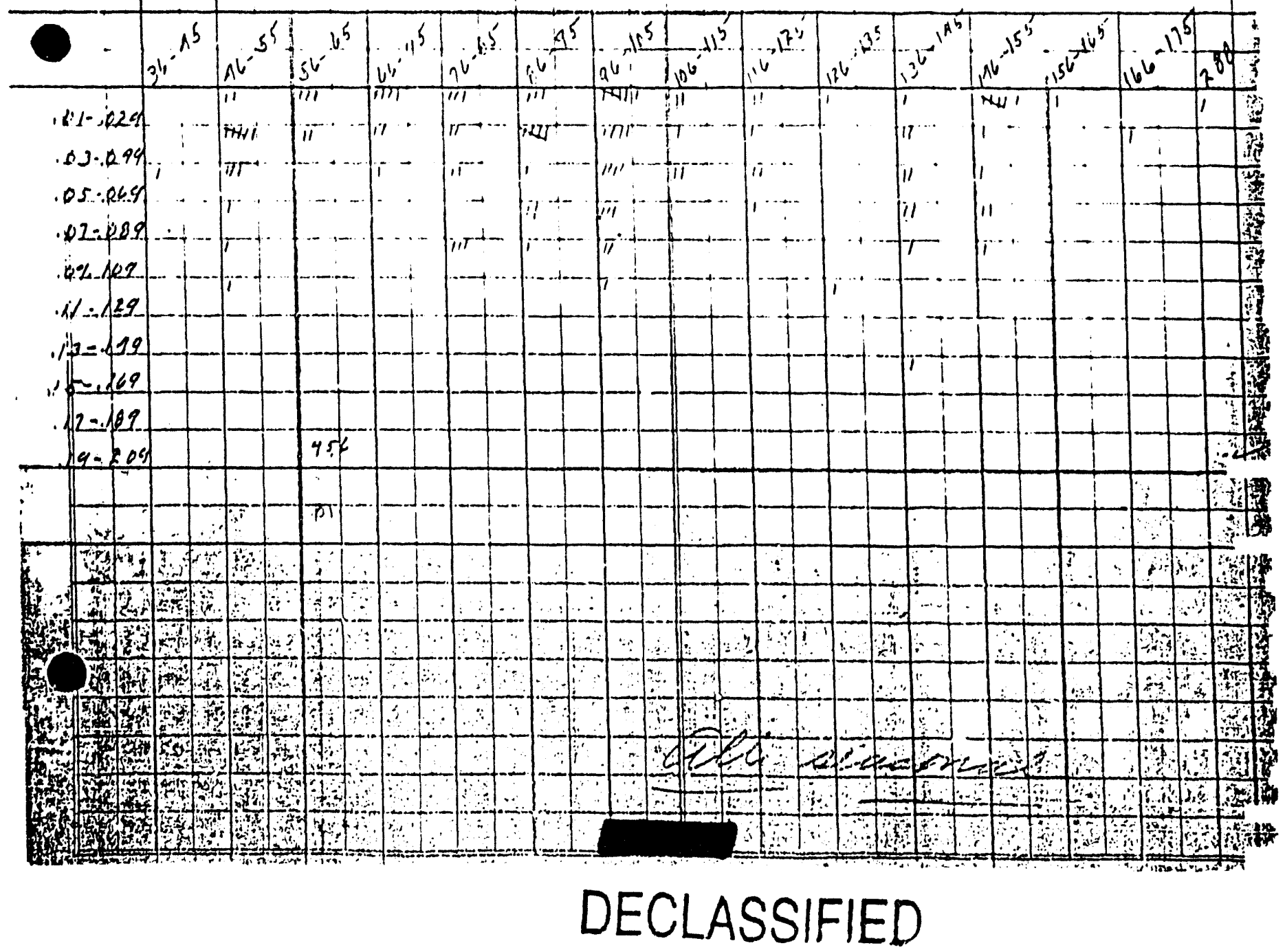




\section{DECLASSIFIED}

$\therefore \%$
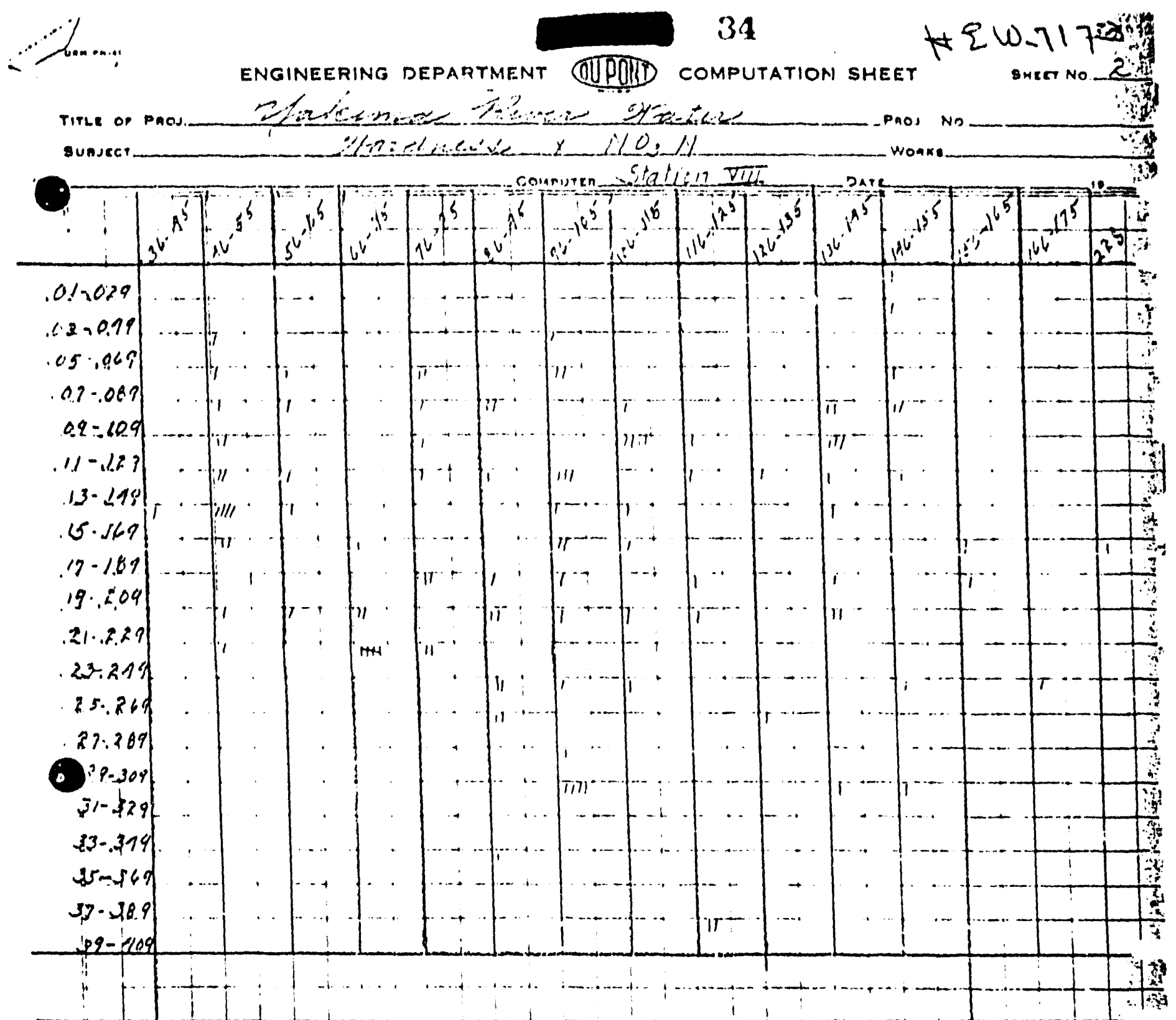

Tirle or PAOs.

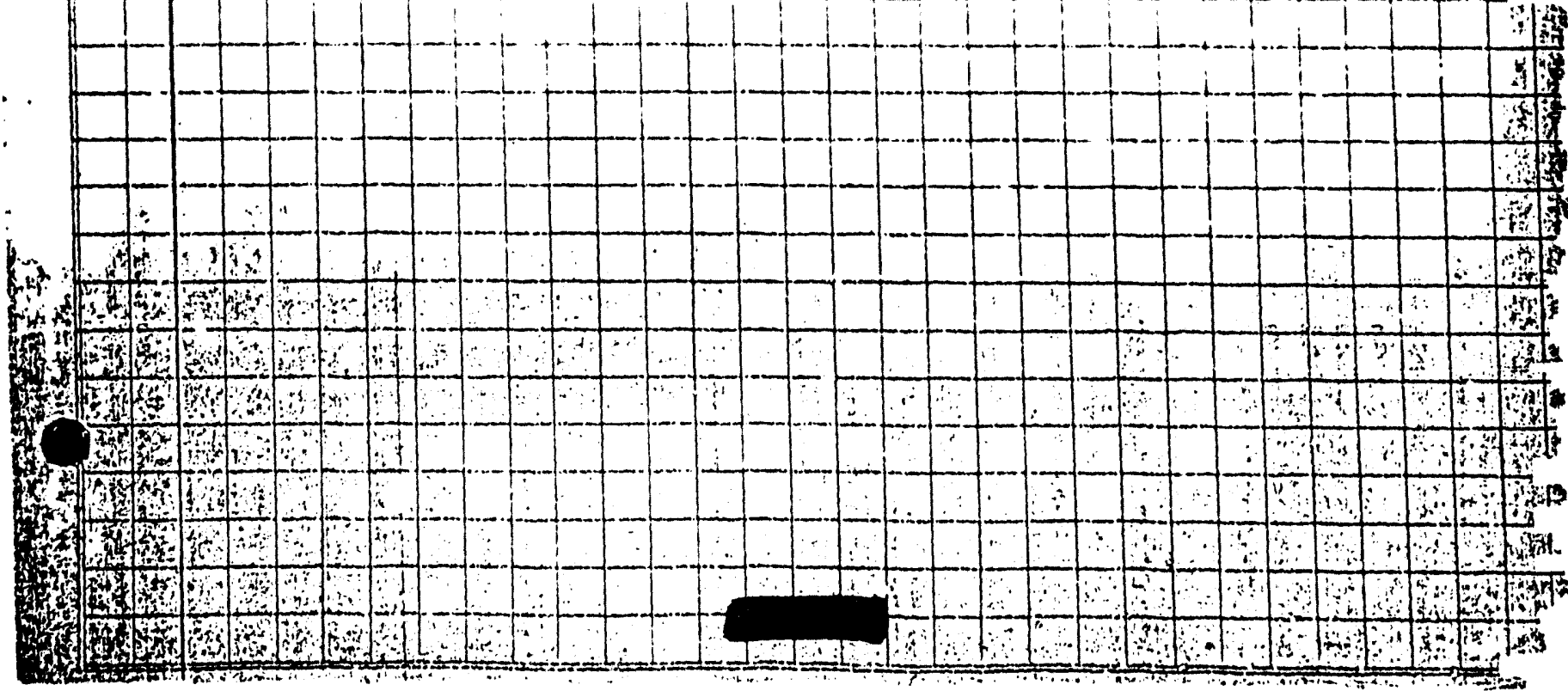

DECLASSIFIED 


\section{DECLASSIFIED}

$\%$

Engineering Department

$H q W .717 \%$

COMPUTATION SHEET

suesinos

TITLC OP PaOs

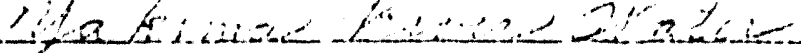

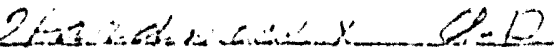

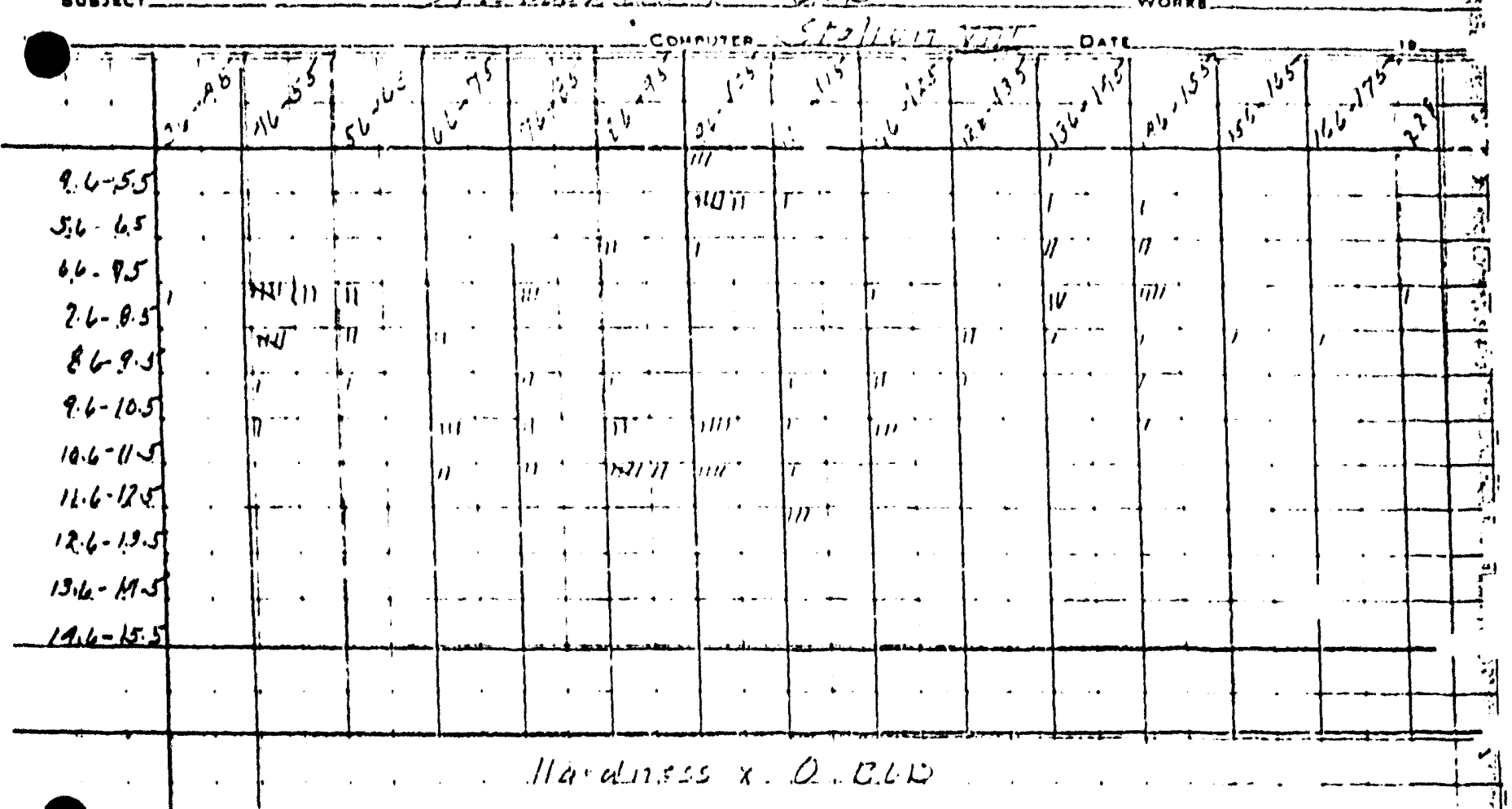

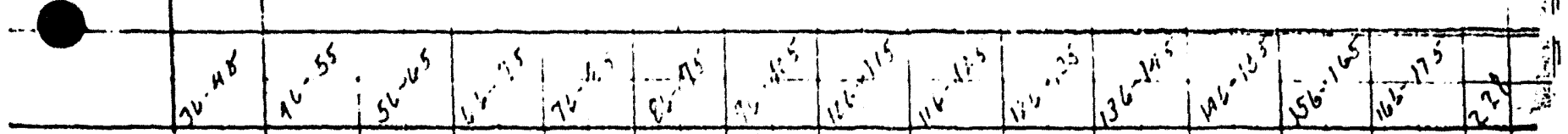

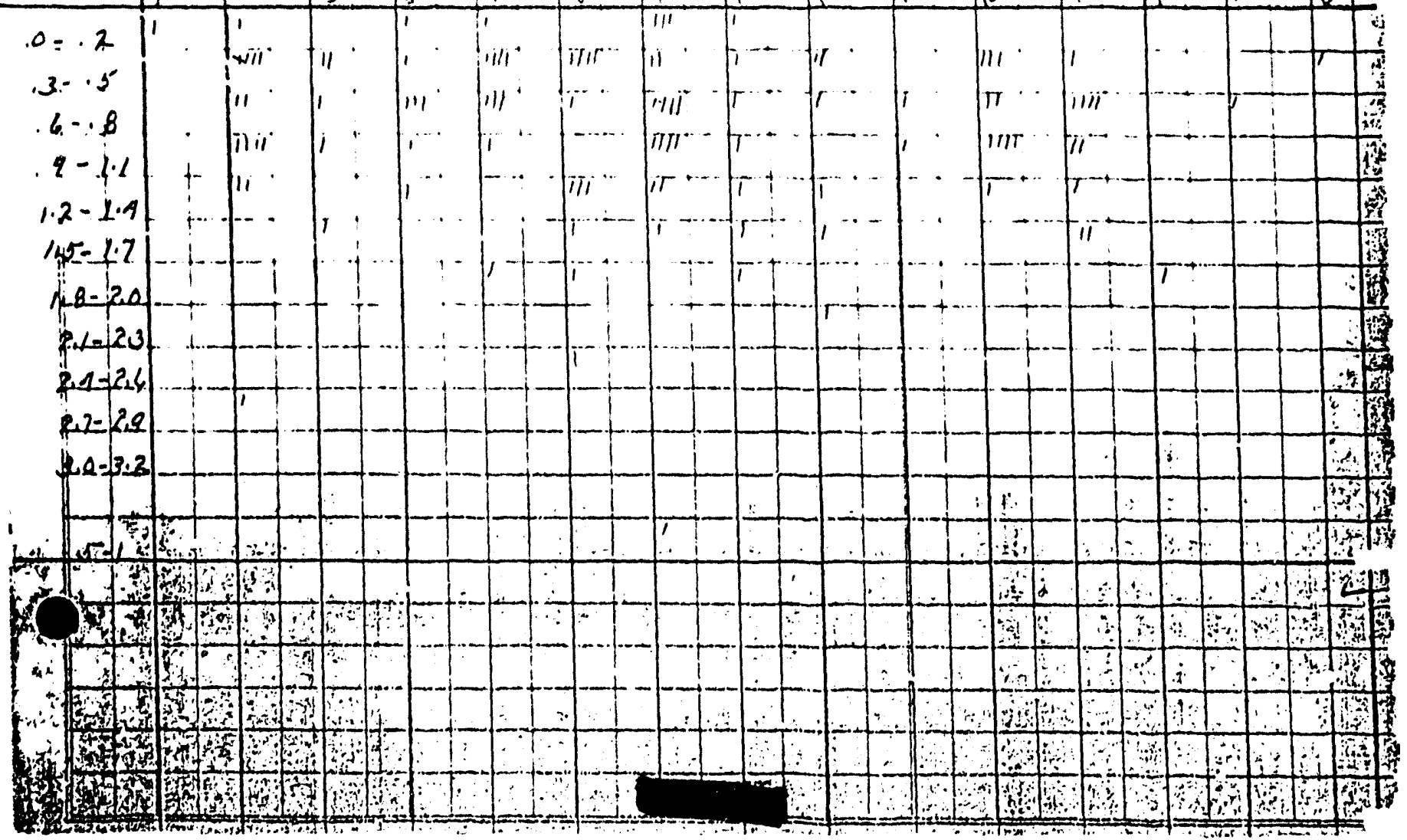

DECLASSIFIED 


\section{미케SSㅂา}
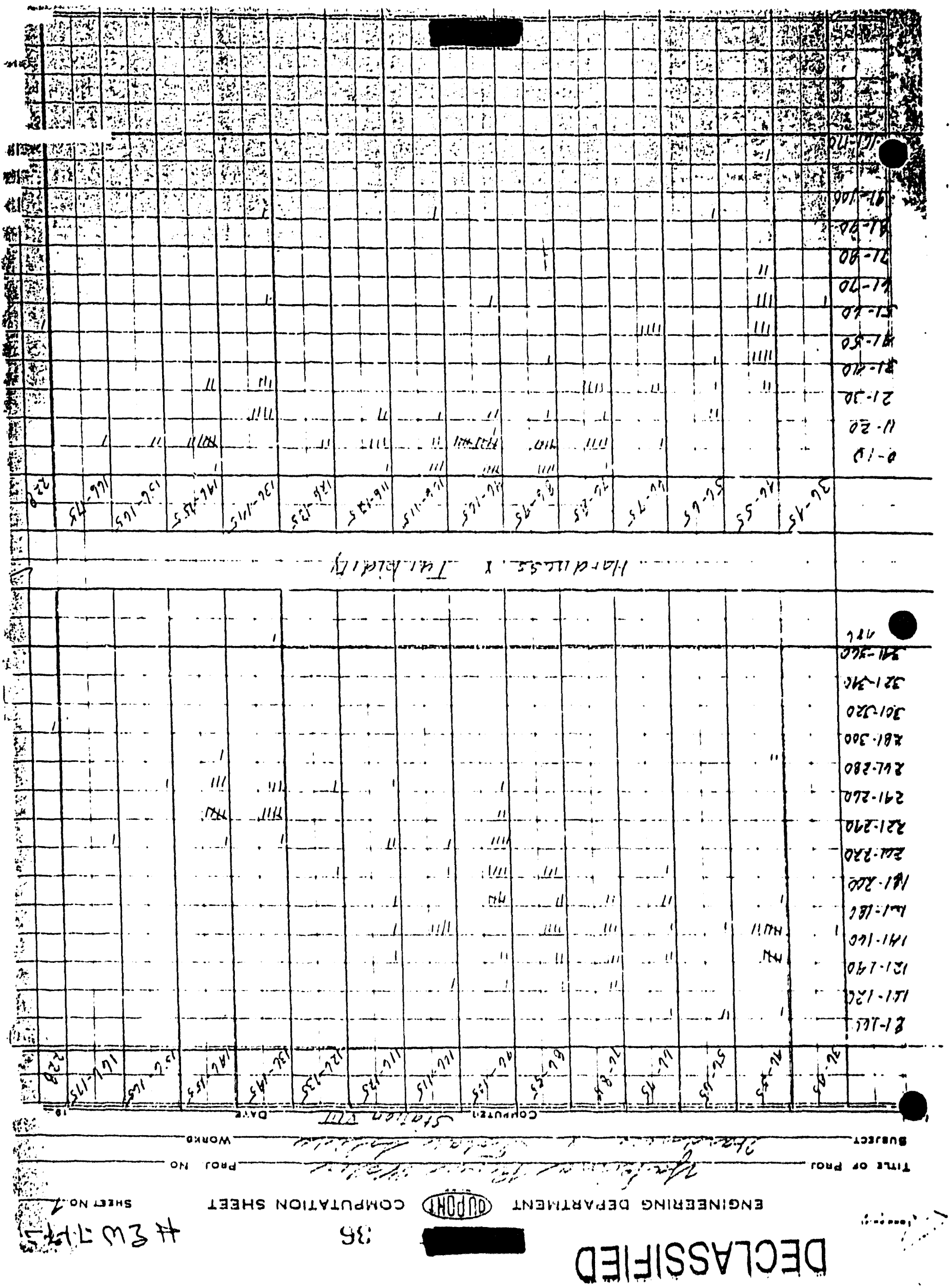


\section{DECLASSIFIED}

ENGINEFING DEPARTAENT OUIDOHD CORAPUTATION SHEST

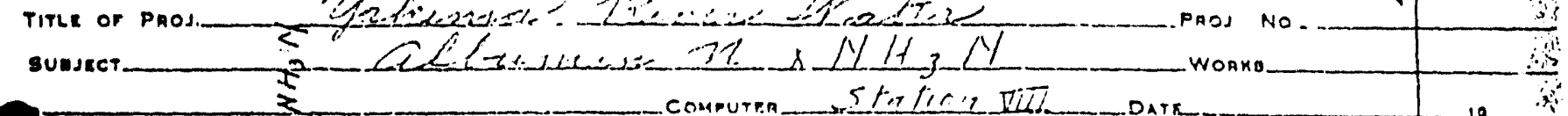
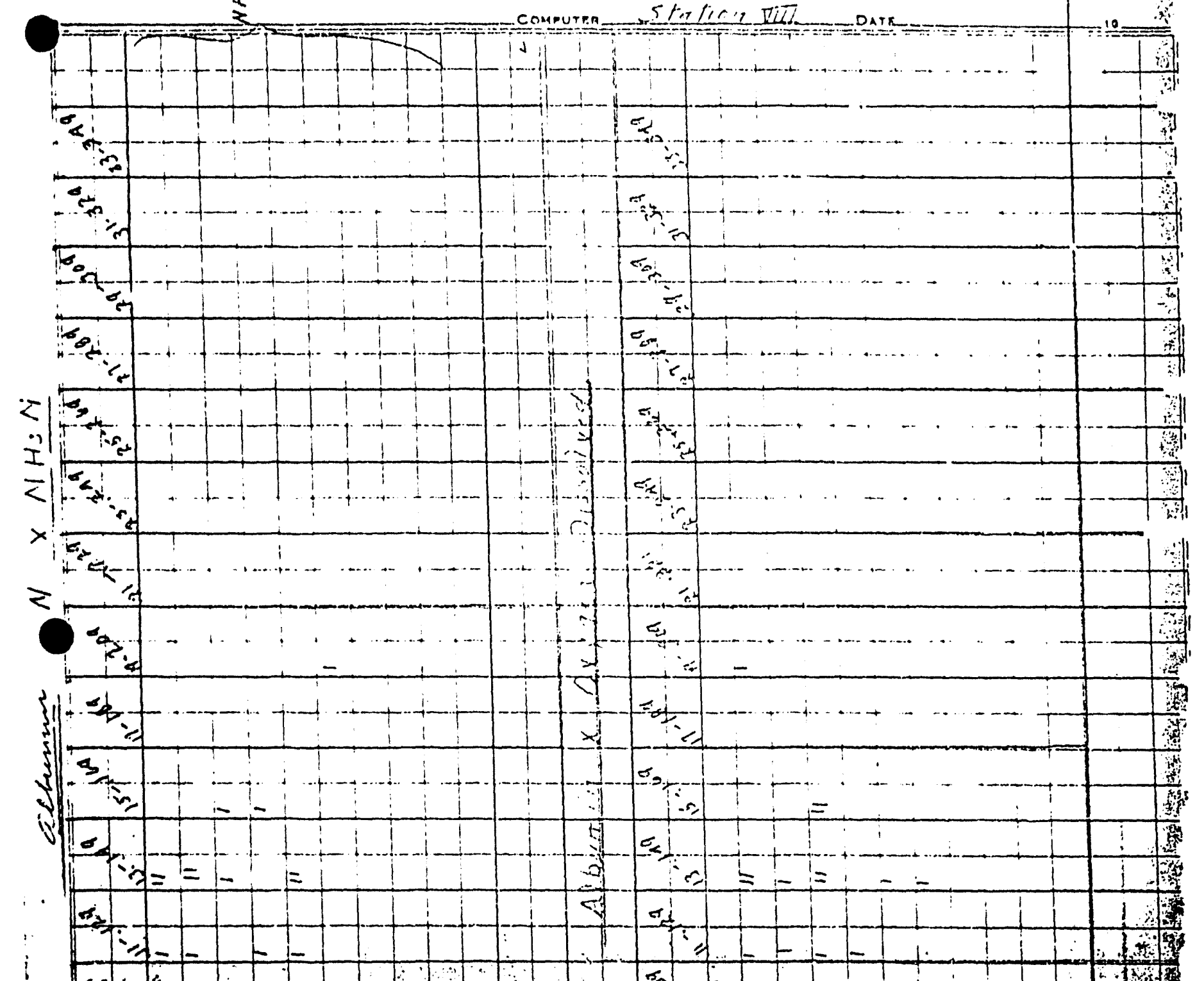

i.

$1 \alpha_{0}=0 \equiv$

\section{i.}

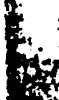

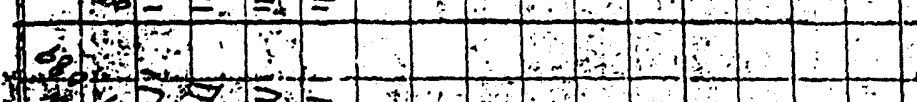

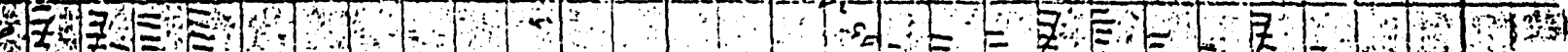




\section{DECLASSIFIED}

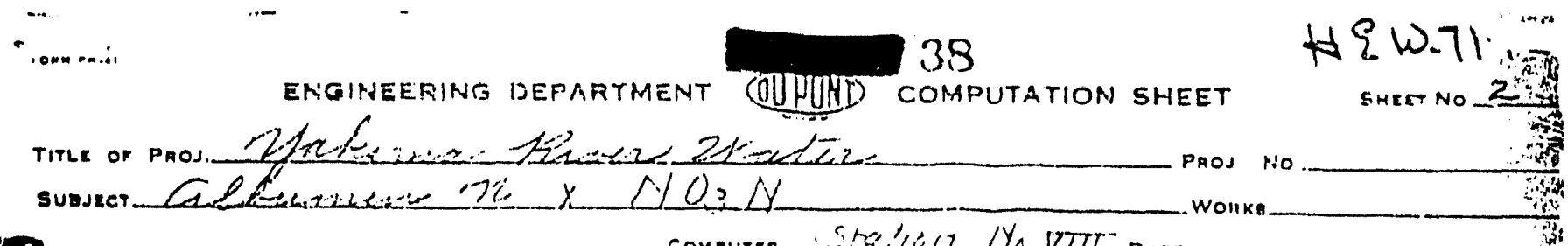

* $=$ =
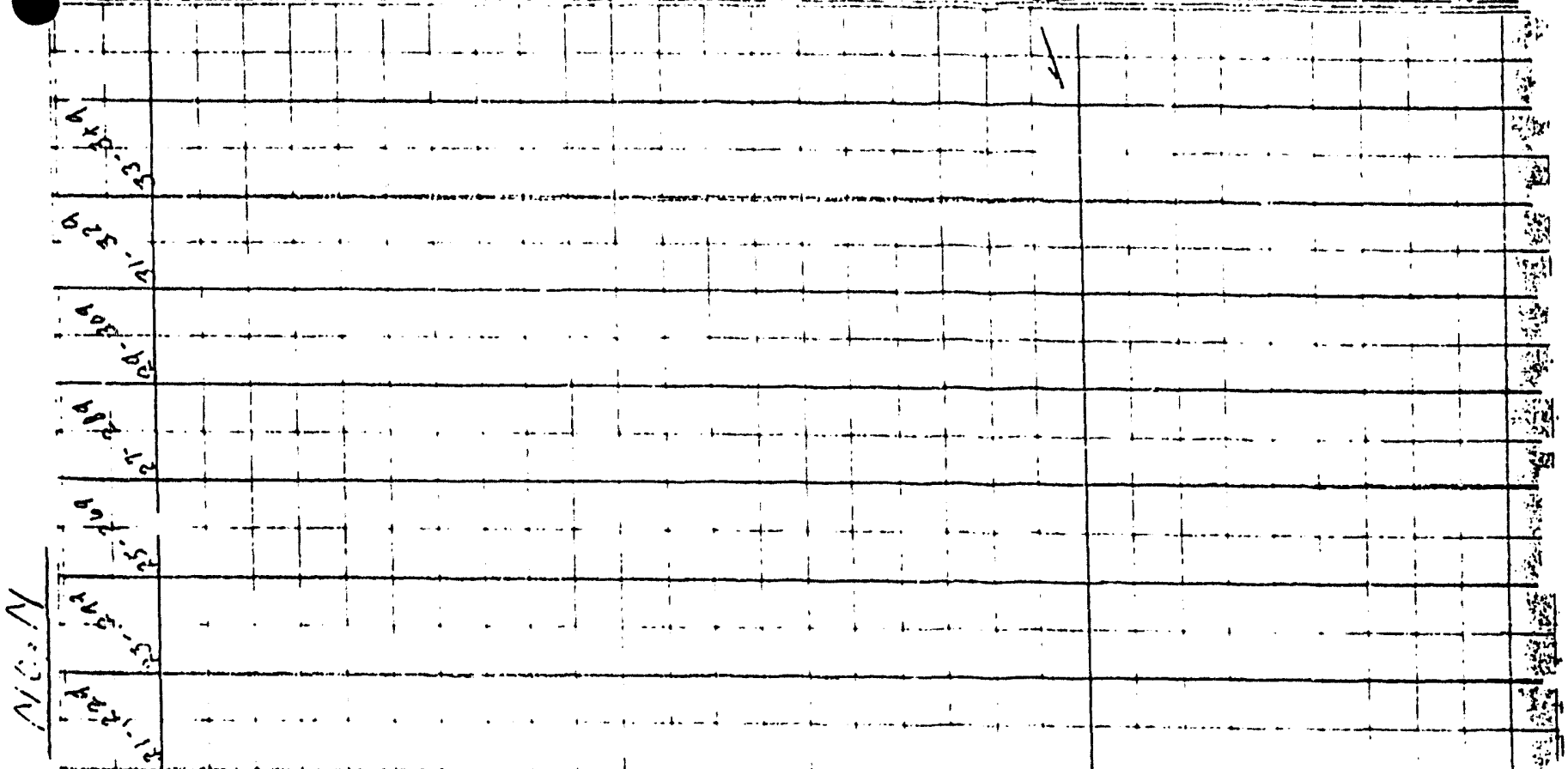

$+\infty$

a

.

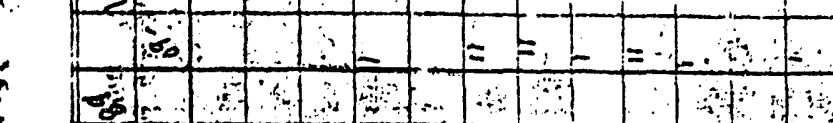

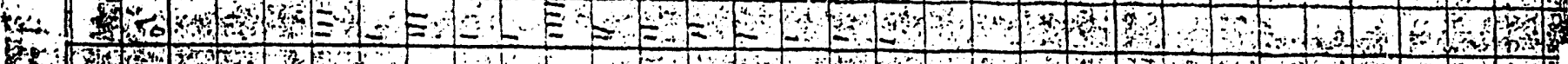

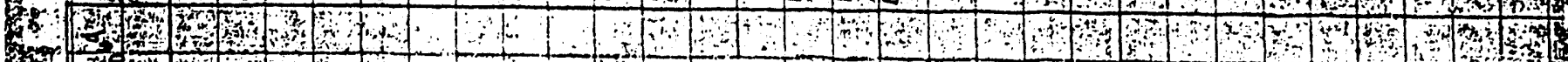

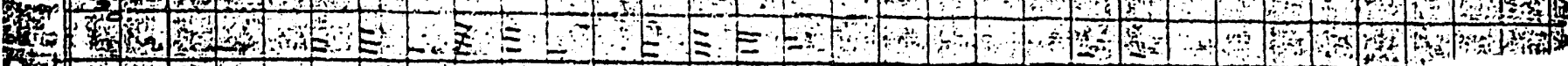

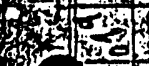

L $1=0$ -

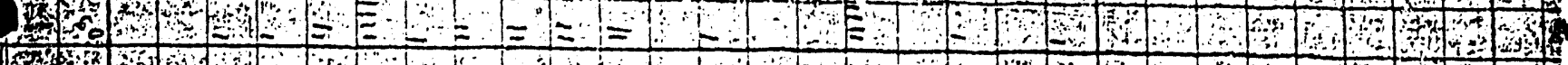

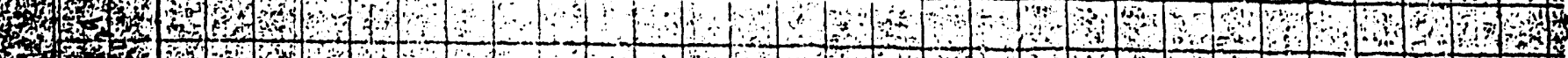

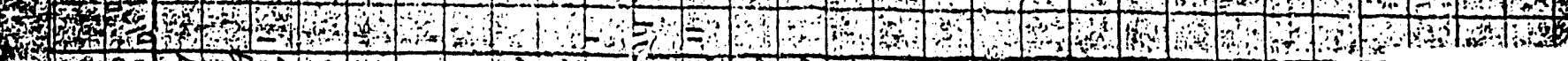

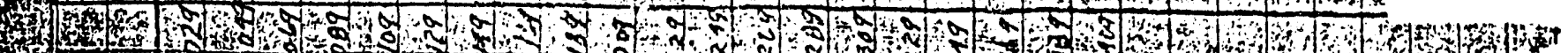

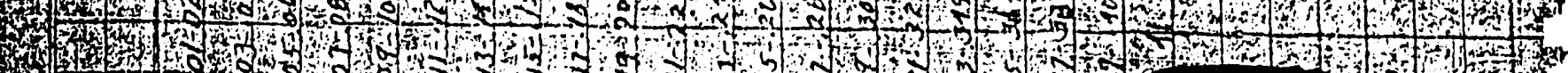




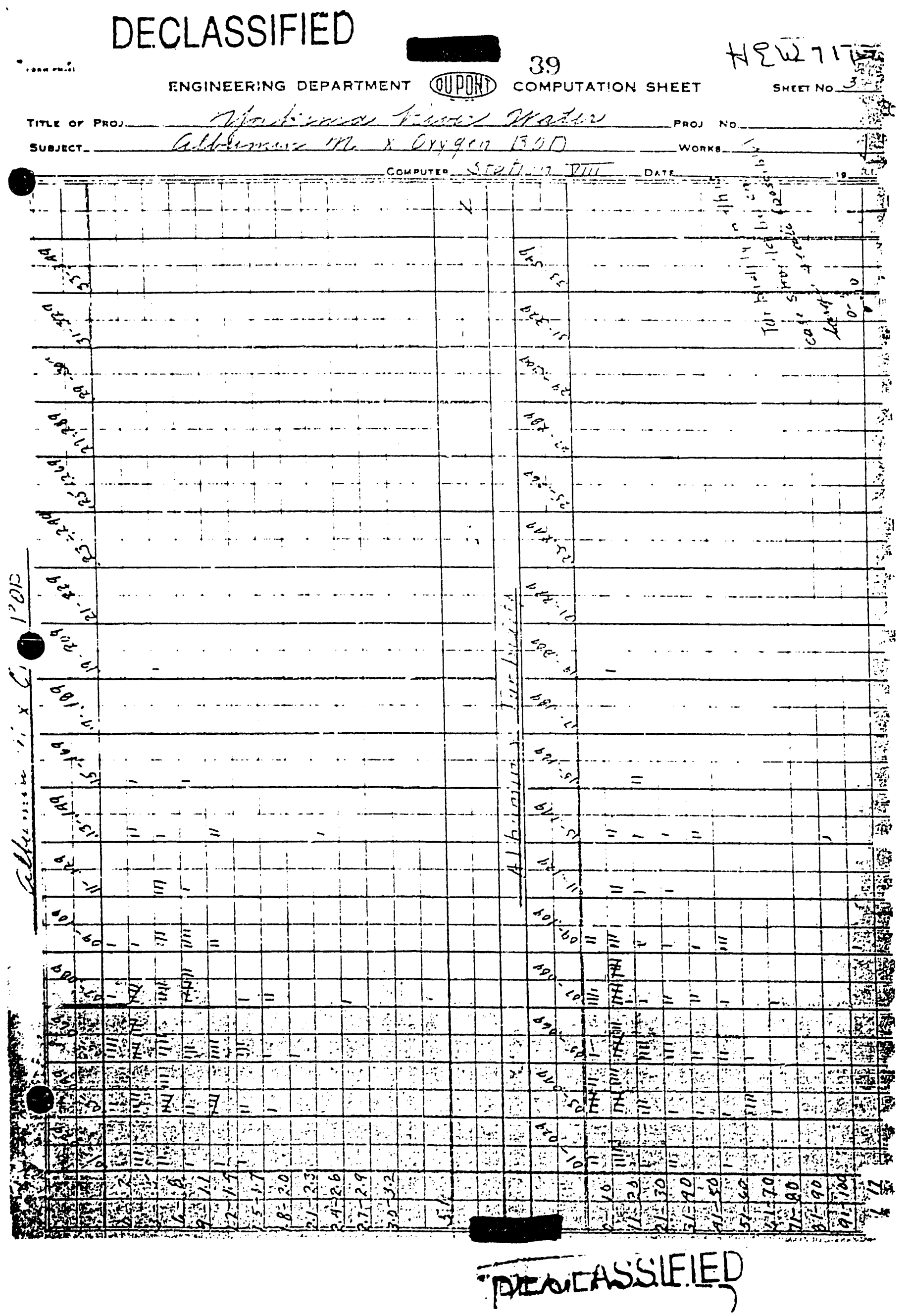




\section{DECLASSIFIED}

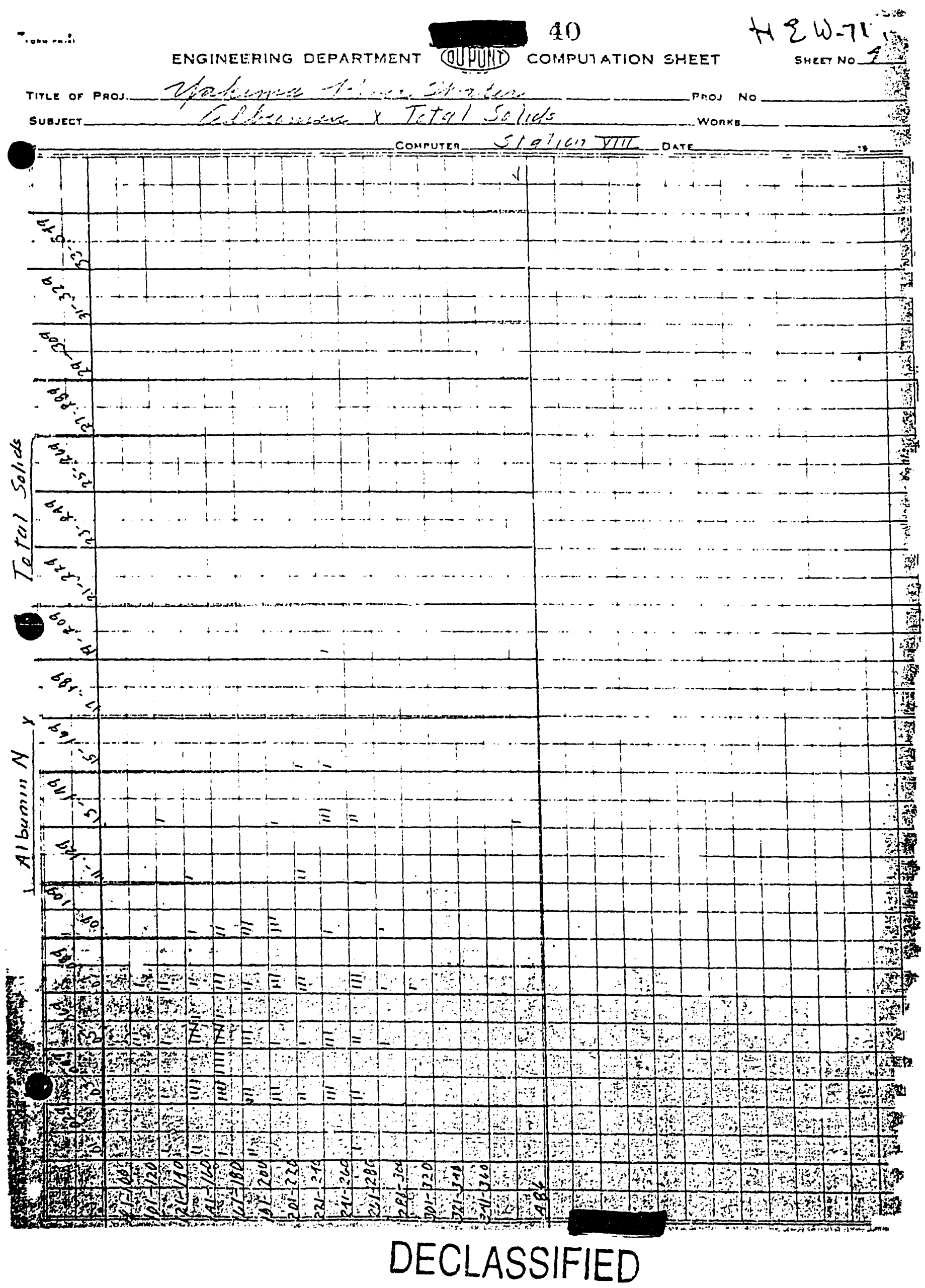




\section{DECLASSIFIED}

doam rm.e.

ENGINEERING DEPARTMENT OUIDOHD COMPUTATION SHEET

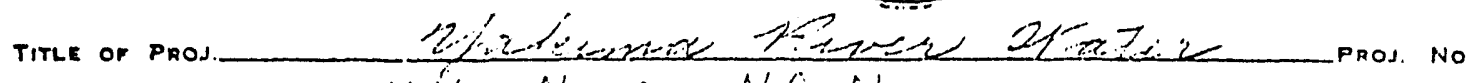

SJIJECT $\Delta H=N-A \quad N S=M$ WonkB

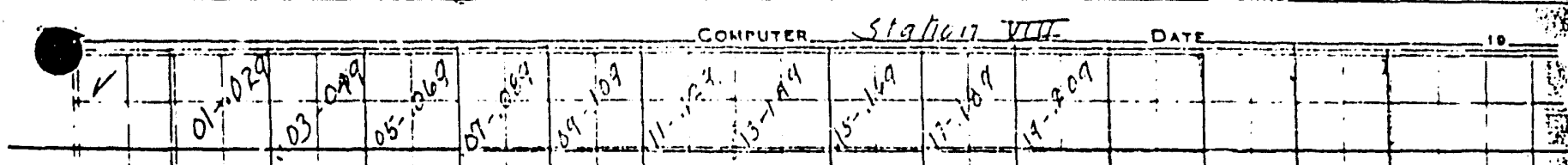

$11-.229$

$0 .-4.29$

$02.8<9$

a). -bos

$.09=\mathrm{Keq}$

$w=229 \pi+\cdots$

|k-2.19|

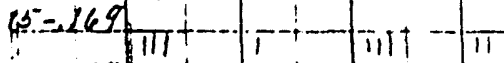

$47=-189$

.$\$ 9 \cdot 202$

स. $2 \times 29 \pi+1 \pi+-11$

मी $^{3}=249$

25-zed

, $27 \cdot 289$

- 24,309

$131-3,29$

23:-314

$.35=569$.

$27-389$

tres-109

a1

.

$+$

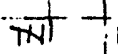

in:

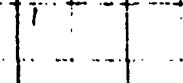




\section{DECLASSIFIED}

- ........ 42 ENGINEEPING DEPARTMENT GUPOND COMPUTATION SHEET $+2 \omega-71 \mathrm{~m}$

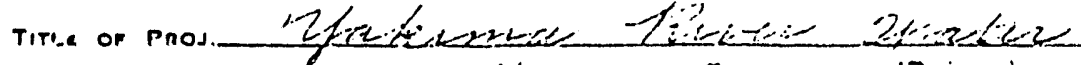

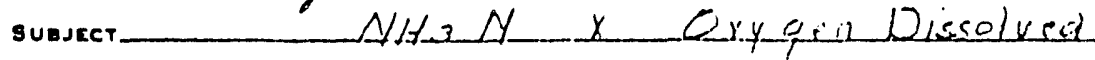
WORKO

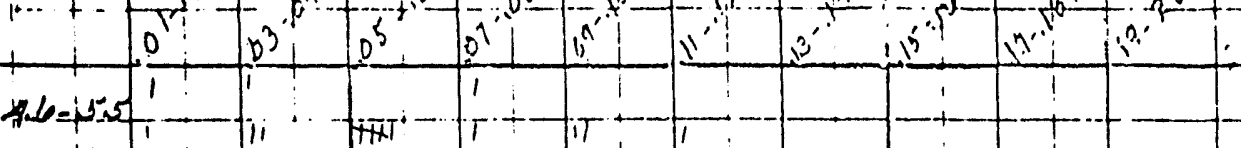
$3,6-2.5$

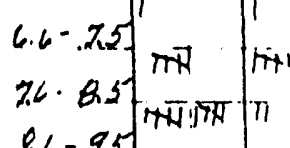
8.6- 255 ind iा

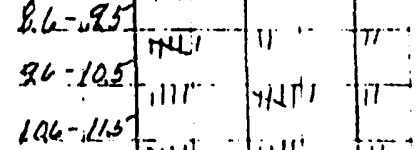
$166 \cdot 2 \pi, 7$. 12.6: $126-11,5$

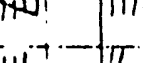
$i^{.}$ (1)....

\section{$1,16-1035$

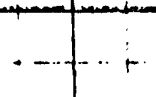

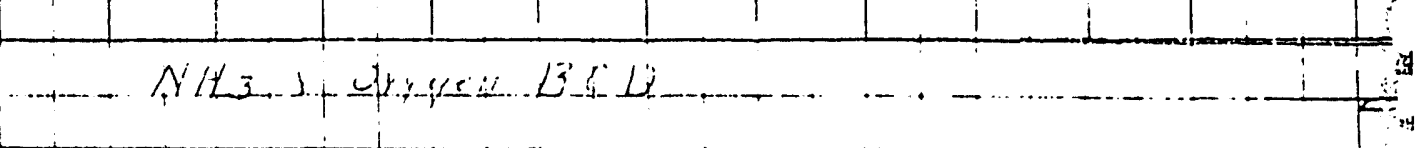

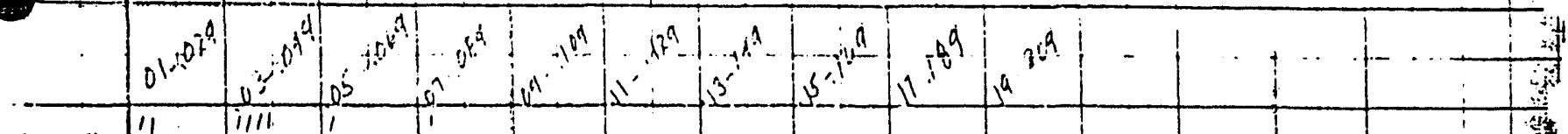

1. . 2

$.6 \cdot .8$

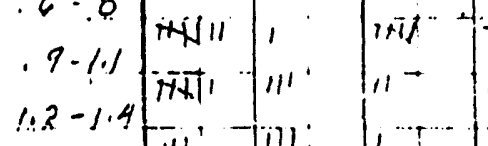
n.5 $5-1 \cdot 7$ $1.8=-3.10$

$2 \cdot 1: 2$.

$2,2-26$ $2.7=2,9$ $-2,8-3.2$

\section{stratsin:}

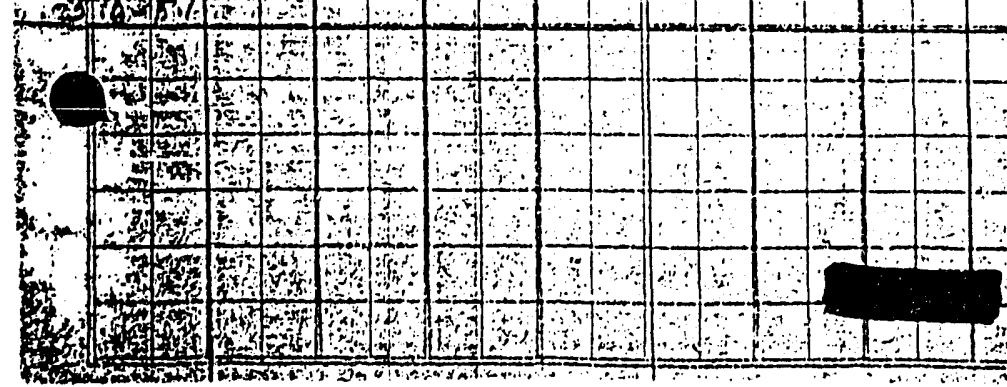




\section{DECCLASSIFIED}

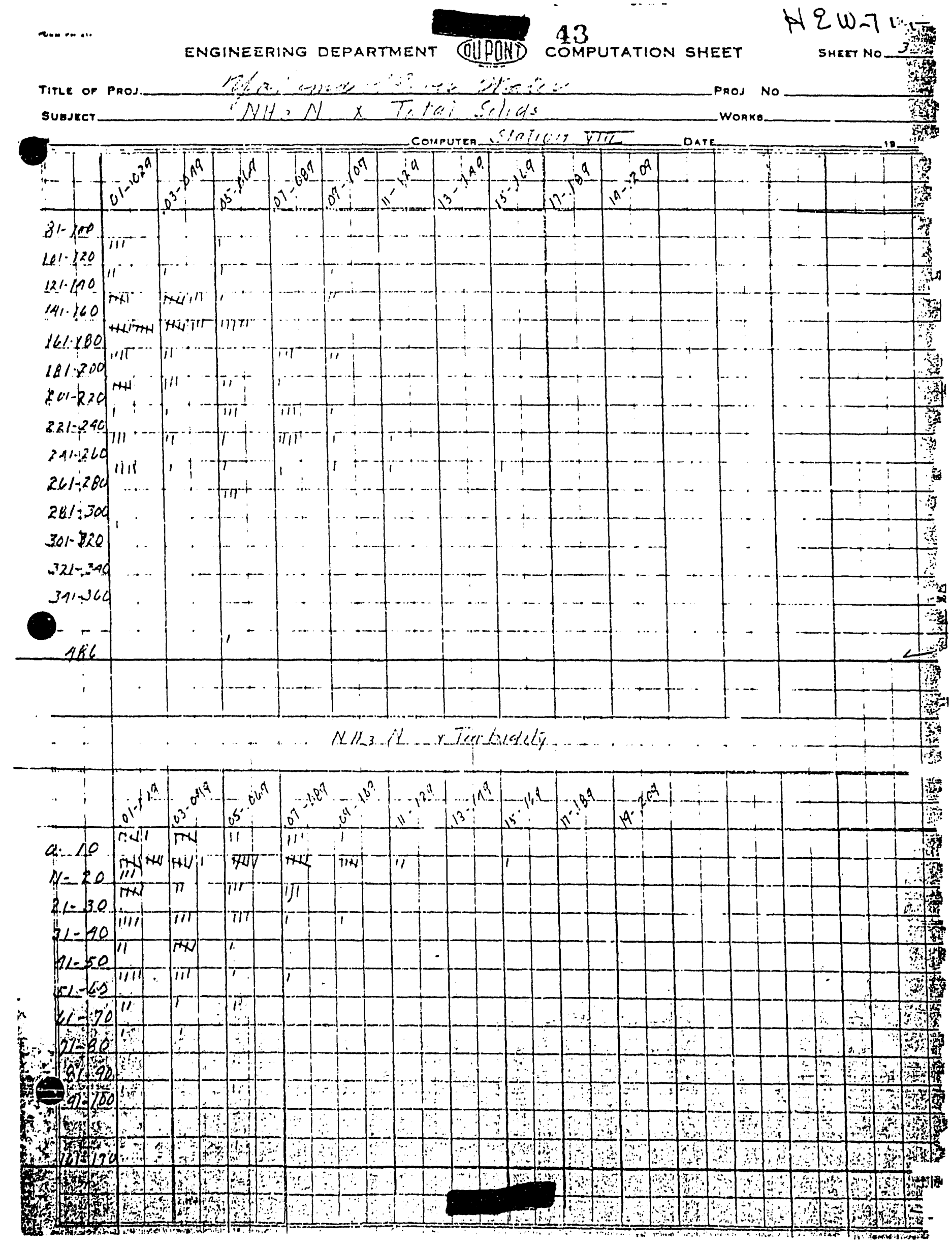




\section{DECLASSIFIED}

-

44

ENGINEETING DEPARTMENT OUPONDD COMPUTATION SHEET

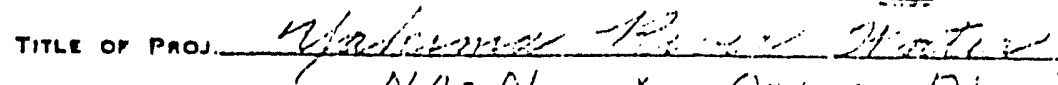

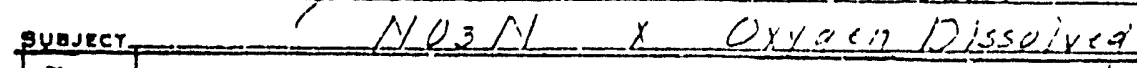

$H \varepsilon \omega_{-i}$

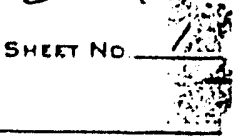

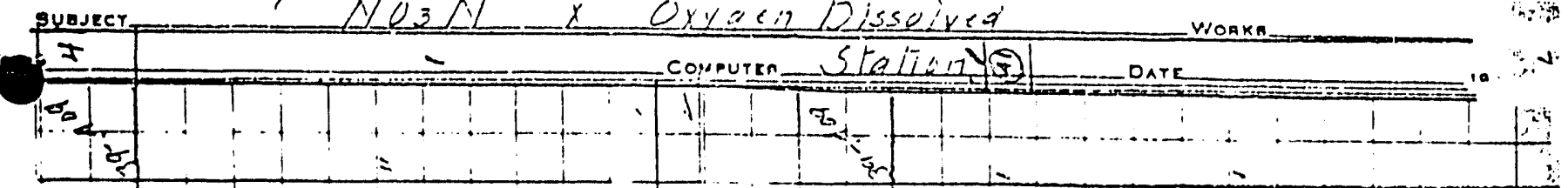

\section{in

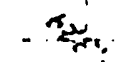

|

(1)

$+1$

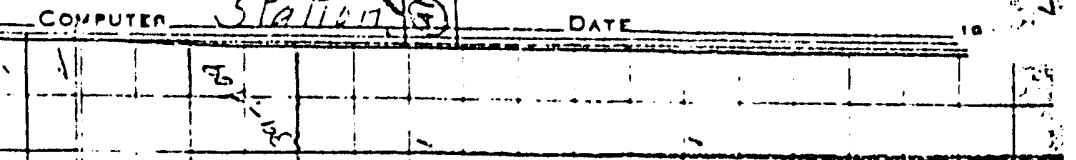

2 -

$1+1-1+2=$

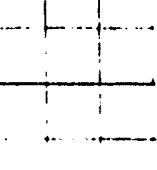

0 is

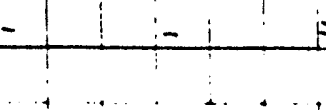

i

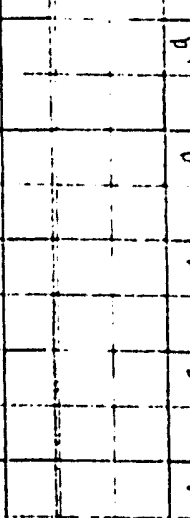

$\Rightarrow$

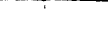

(a)

5

$+-1-$

$=$

$=$

$=$

is

$4-$

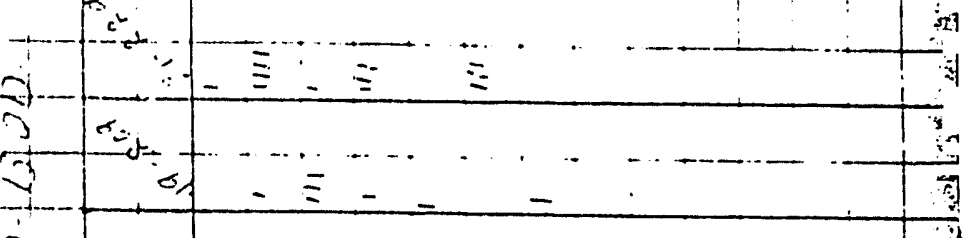

$-\vdots-\cdots$

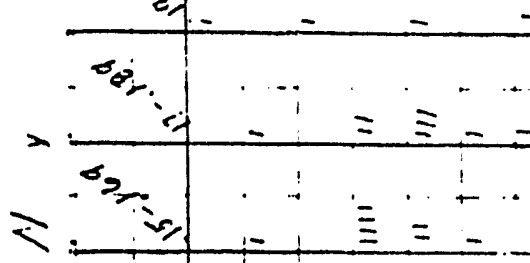

¿n

बर्ये

11

of

E E E

:ंज्ञ $=$ EF

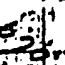

2

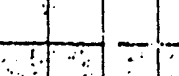

A

120 on

60 a

5: 30 ,

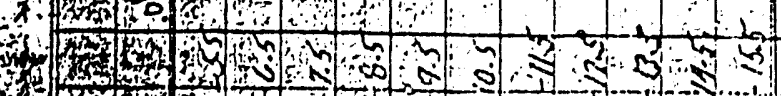

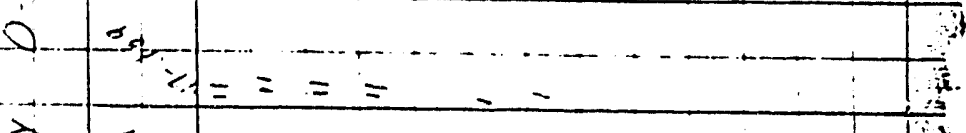

$-$

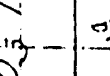

$\because$

27

$\therefore-1-1+1=$

$i_{0}=$

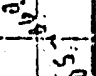

5

$=$

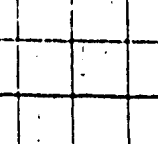




\section{DECLASSIFIED}

Q : :

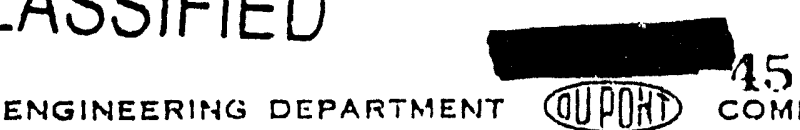

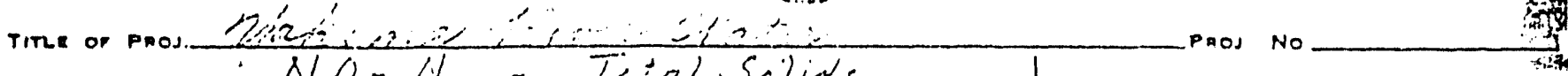

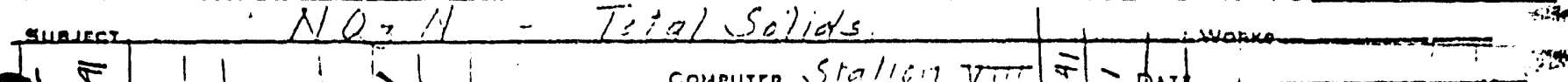

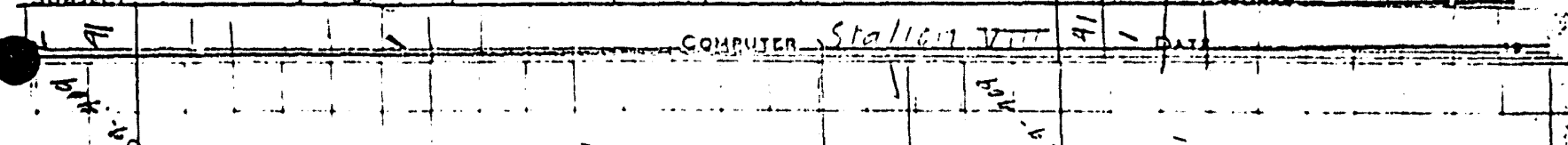

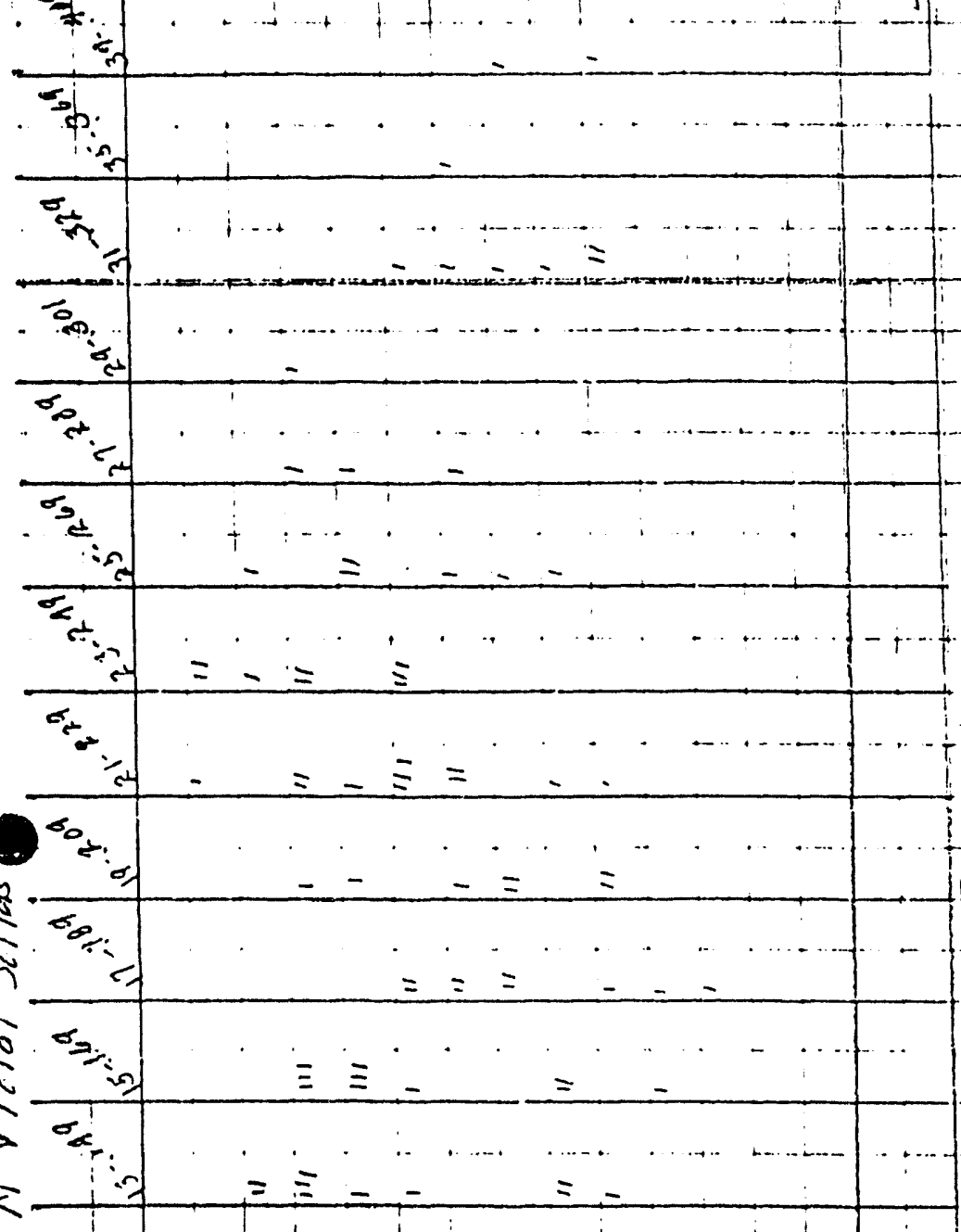

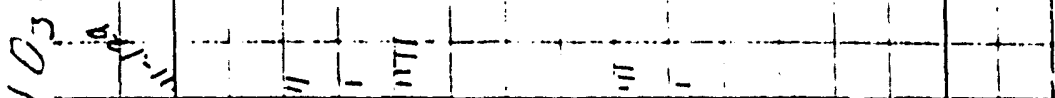
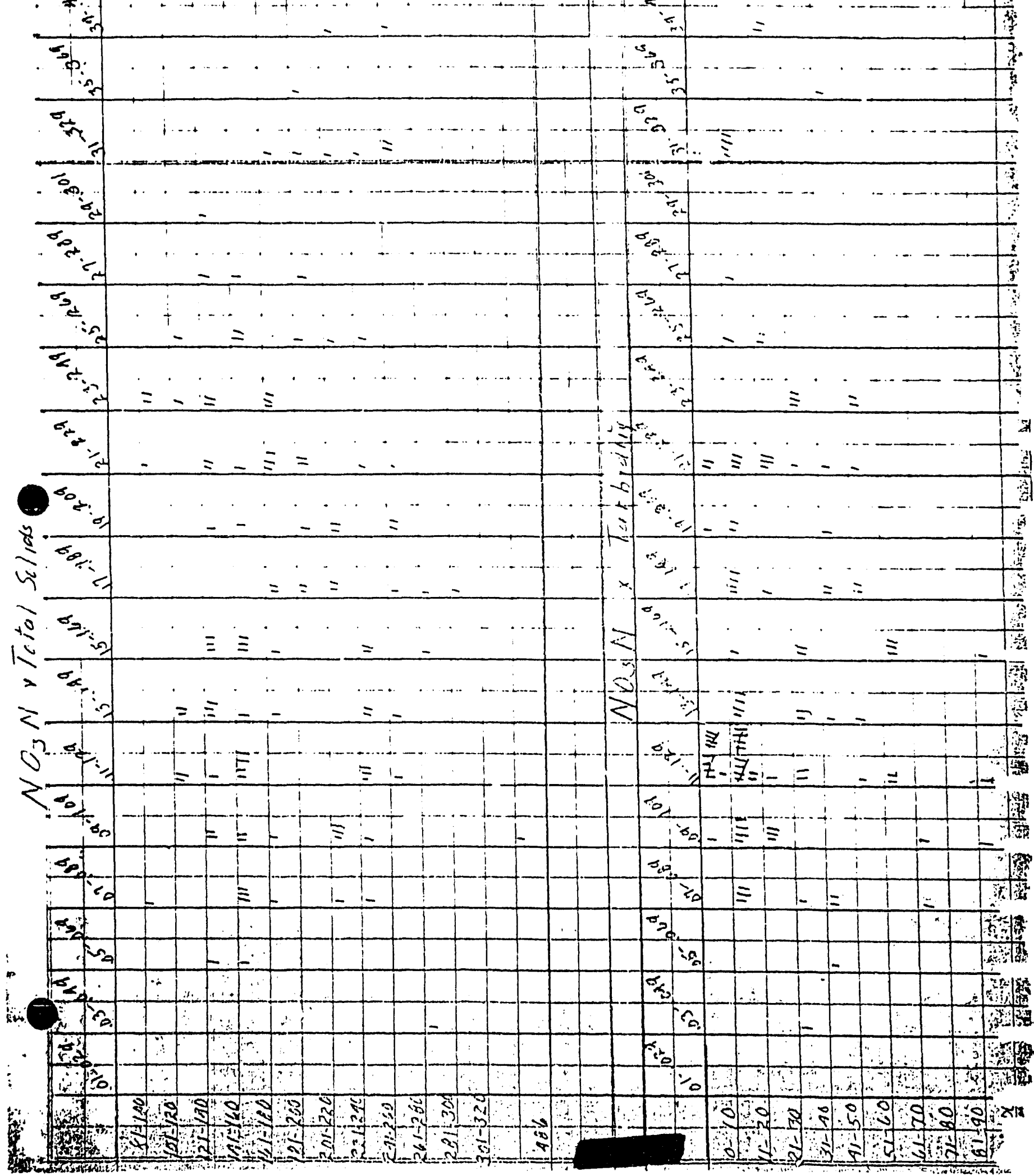
DECLASSIFIED

$-\log \cos$

ENGINEEFING DEFARTMENT

OUPOND COAfPUTATION SHEET

Hqw-i:

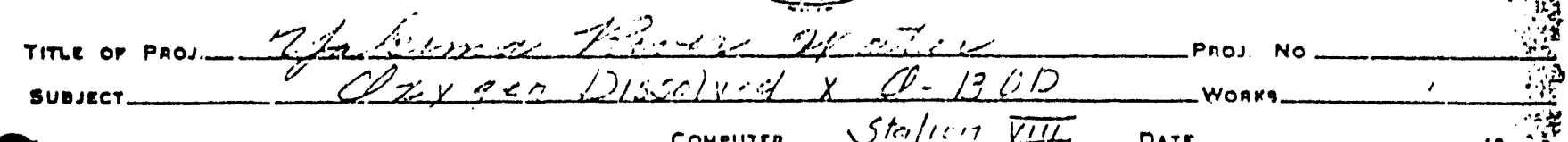

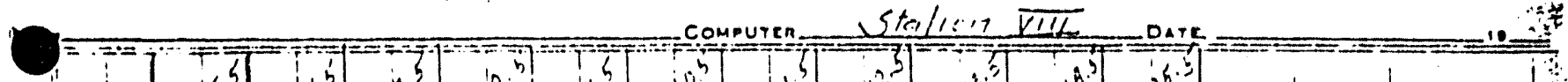

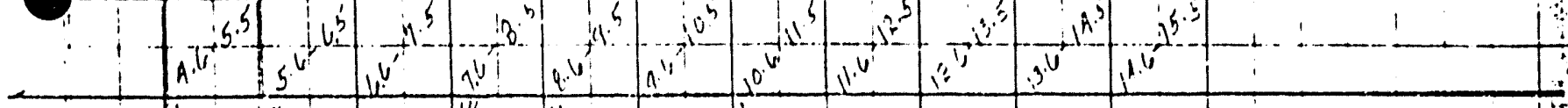

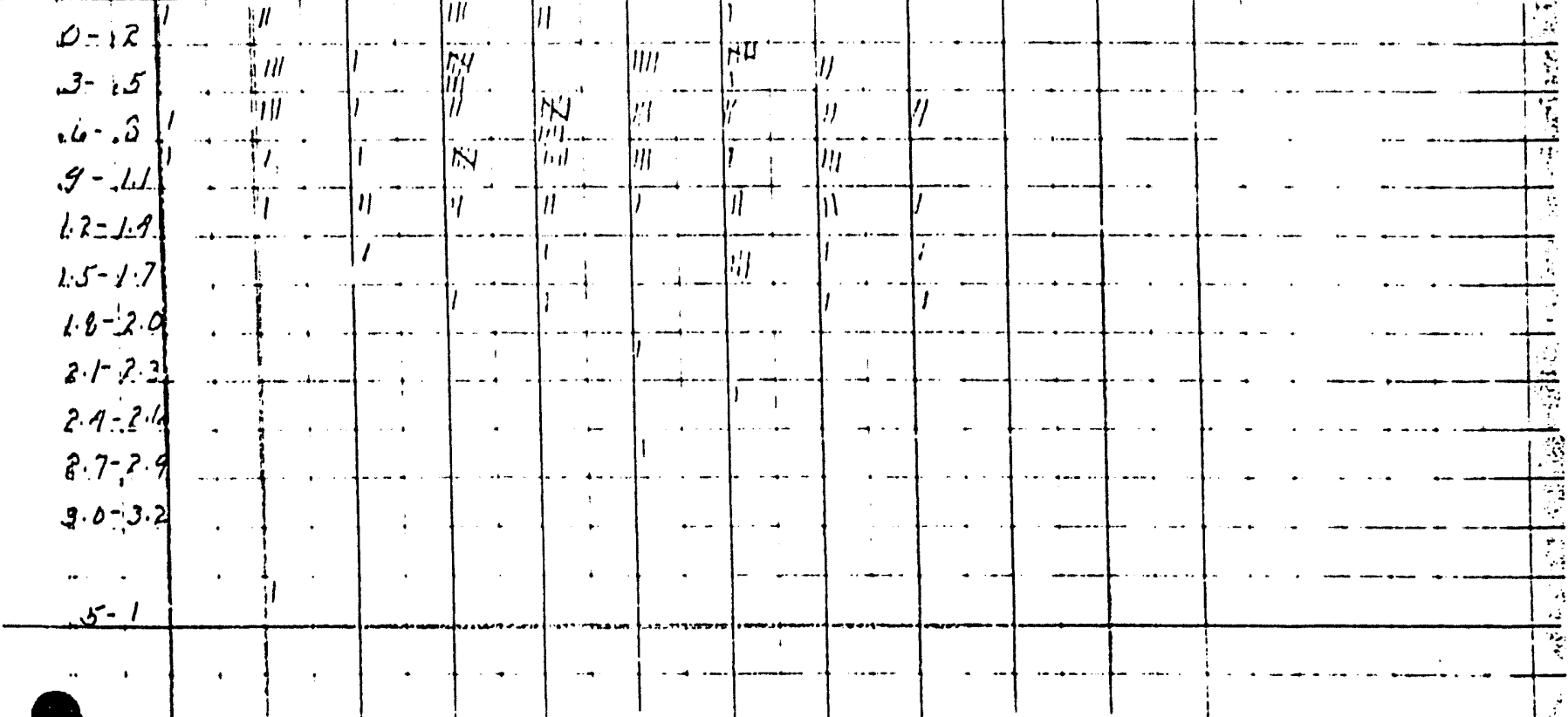

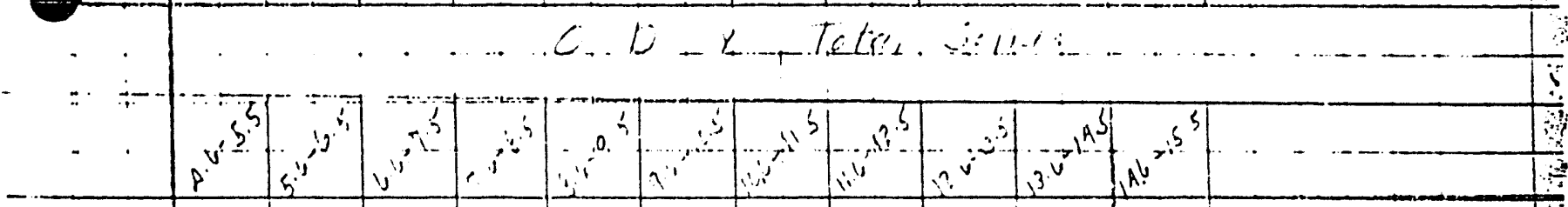

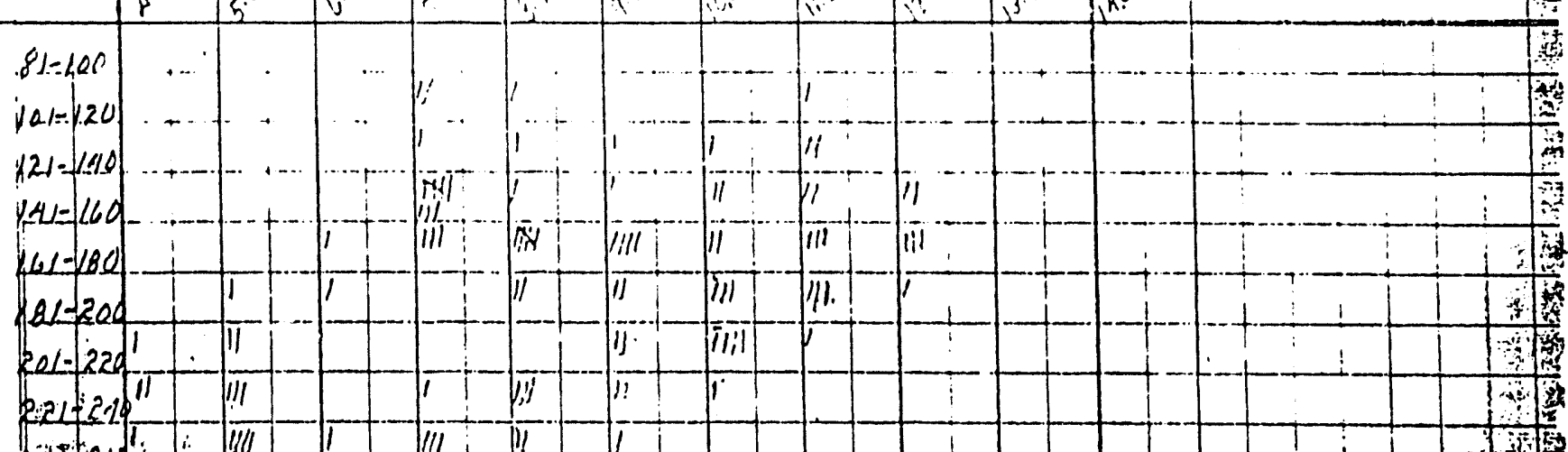

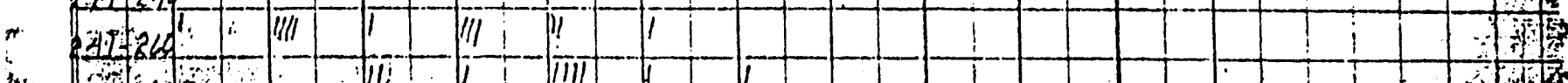

Wat 280

$\rightarrow$ i 


\section{ОᄏІยISS $\forall 70 \exists \square$}

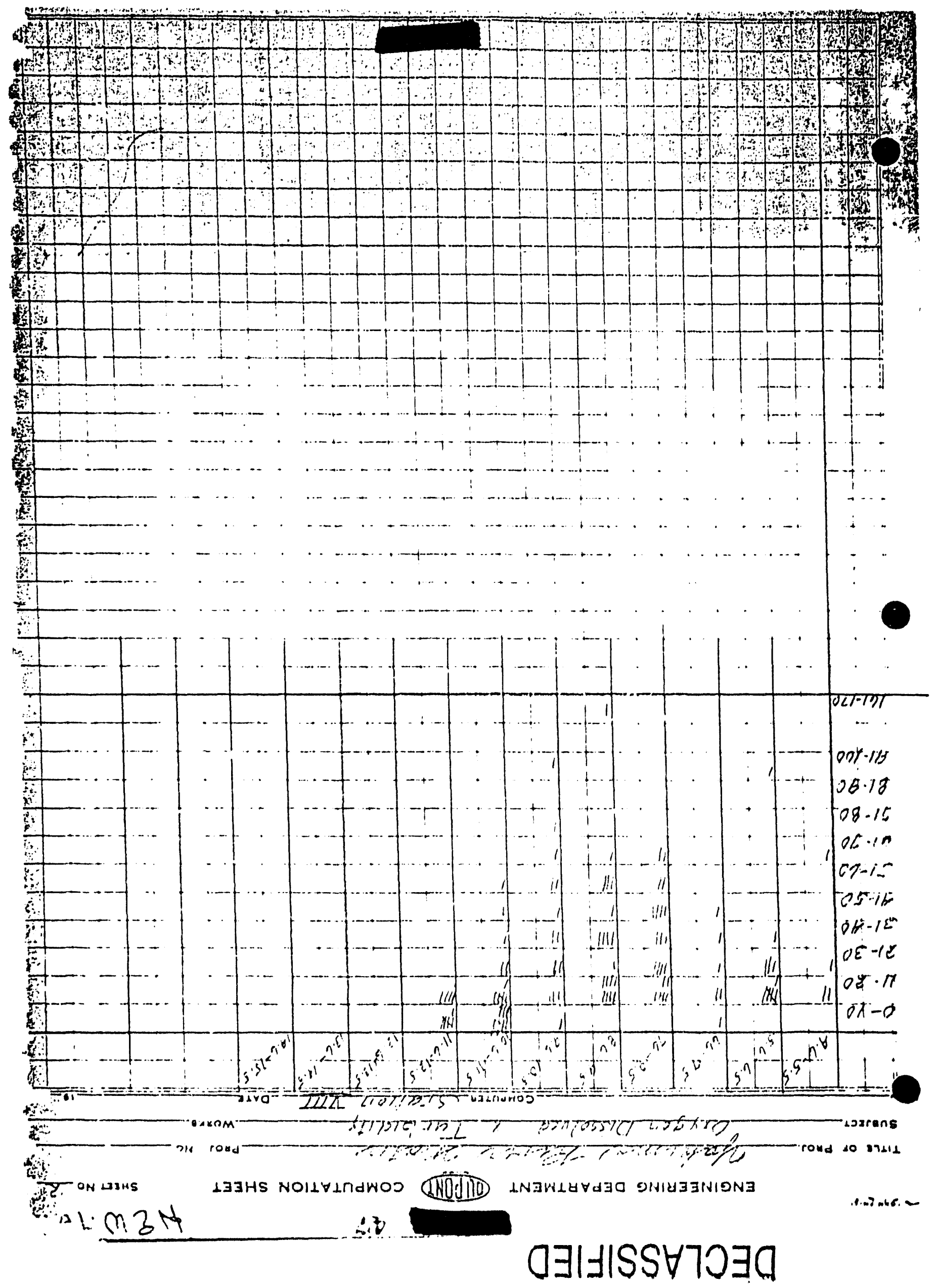




\section{DECLASSIFIED}

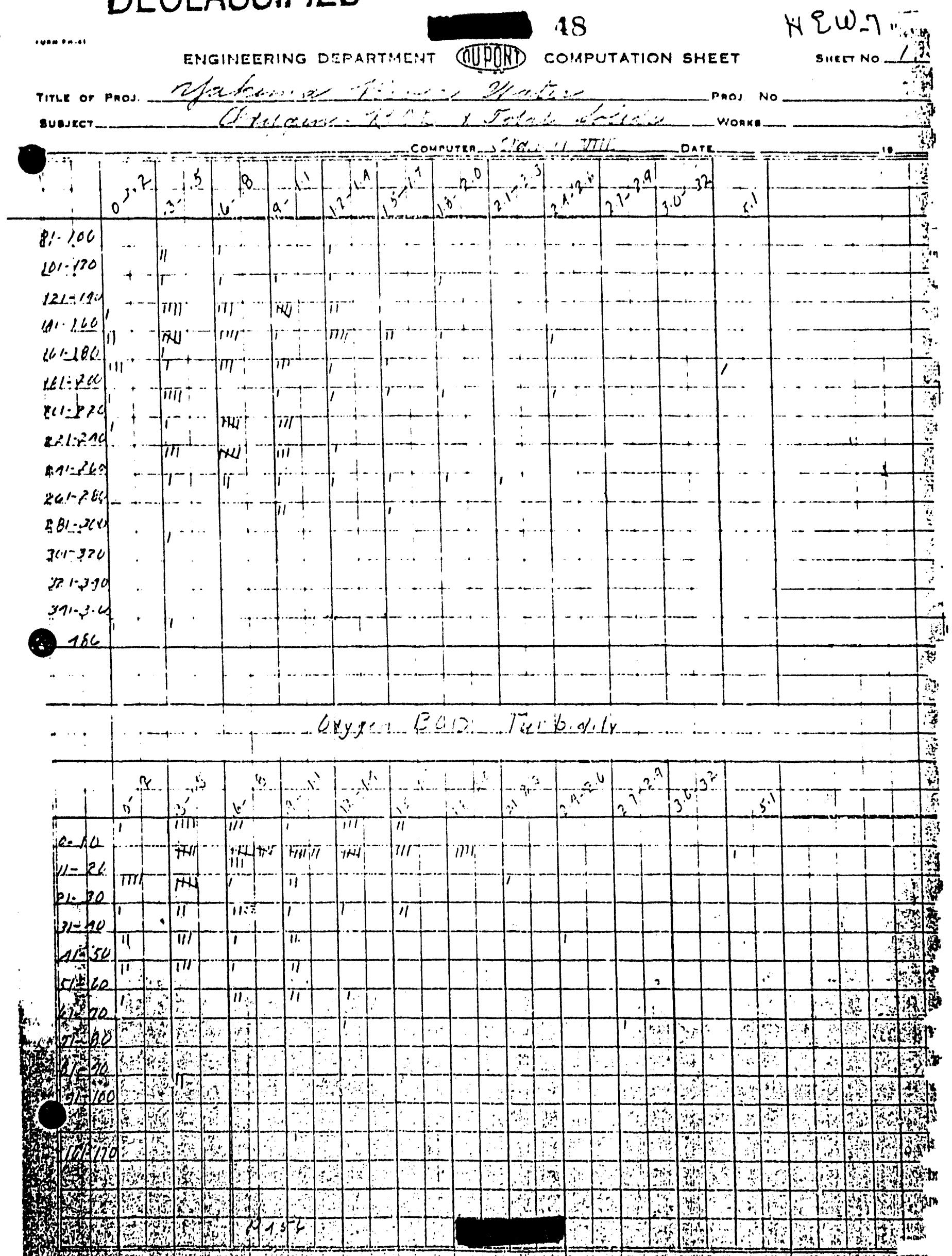




\section{DECLASSIFIED}

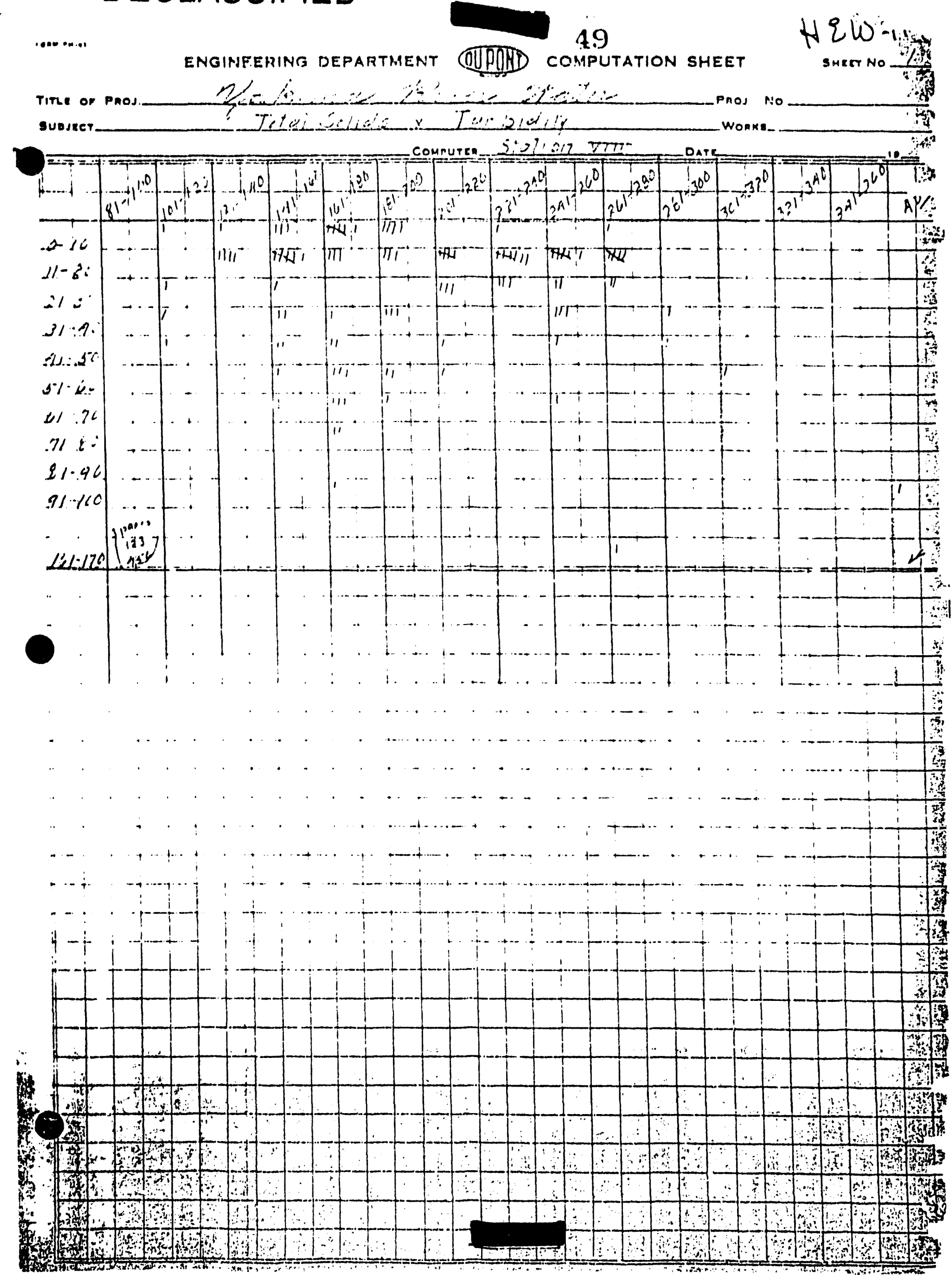

DECLASSIFIED 


\section{DECLASSIFIED}

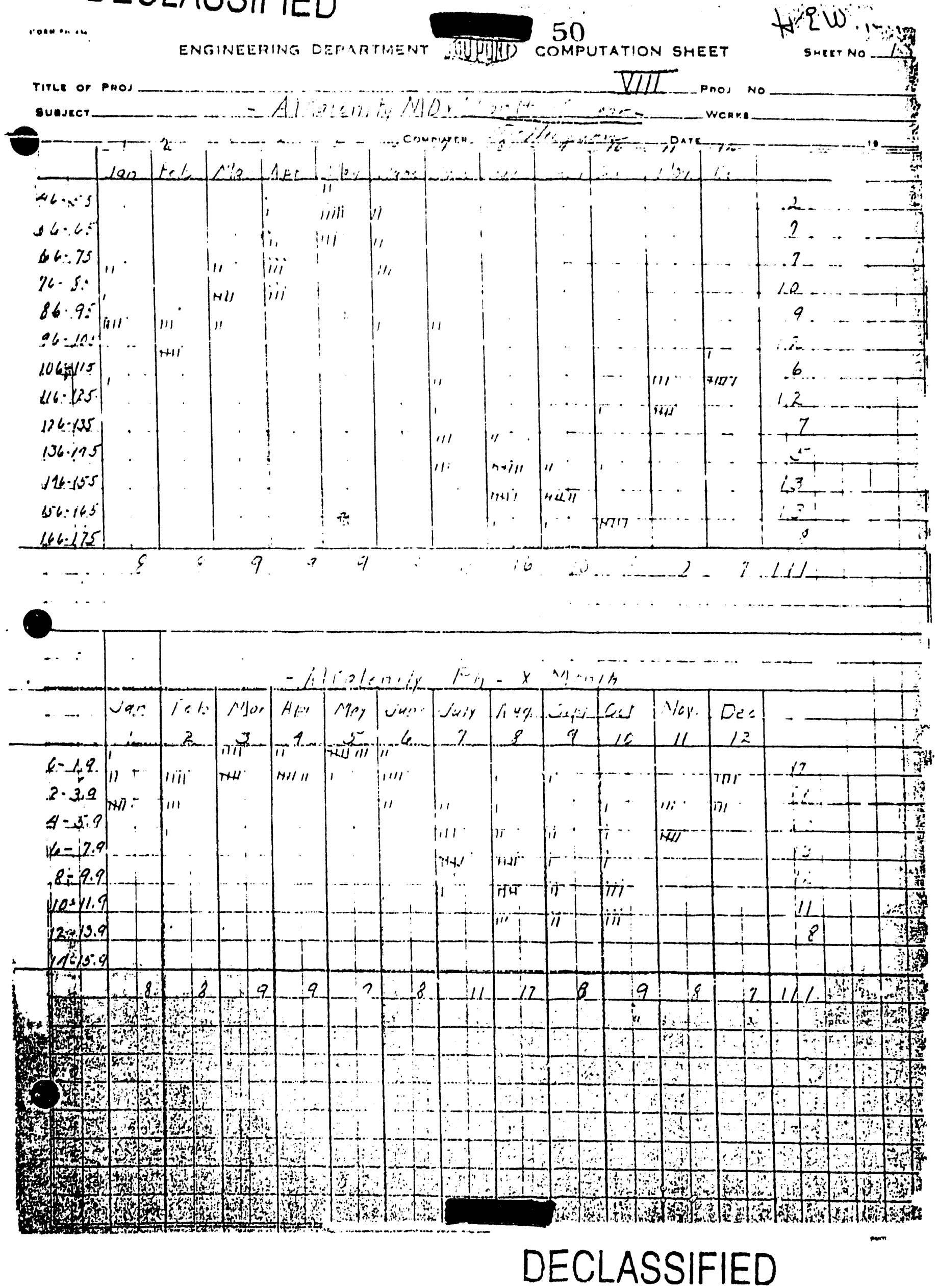




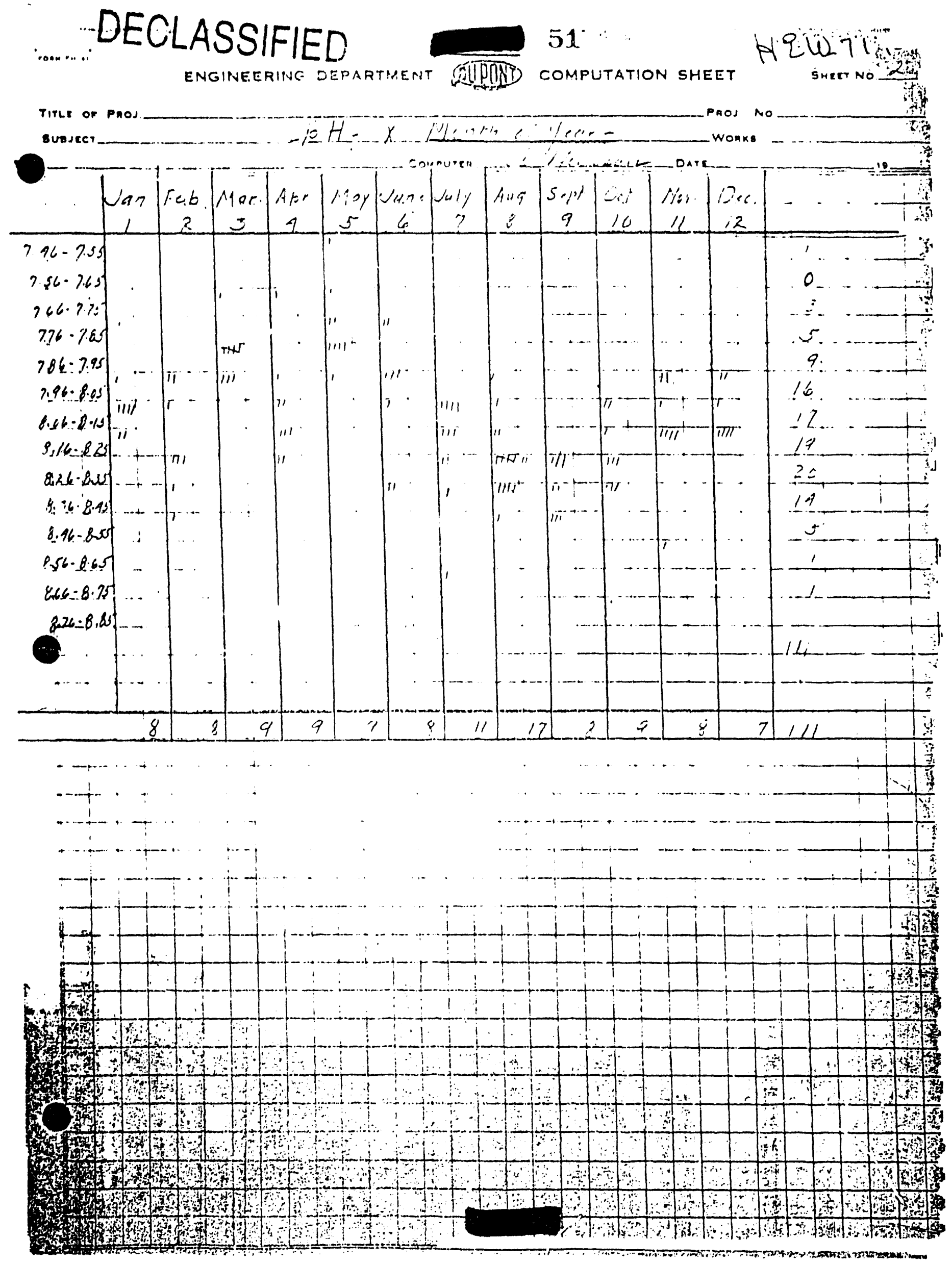

DECLASSIFIED 


\section{DECLASSIFIED}

r.om.....

ENGIREERING DEPARTMENT (TIUPOND COMPUTATION SHEET

TITLE OP PAOS

SUguect Ietol selés y Mlarífear
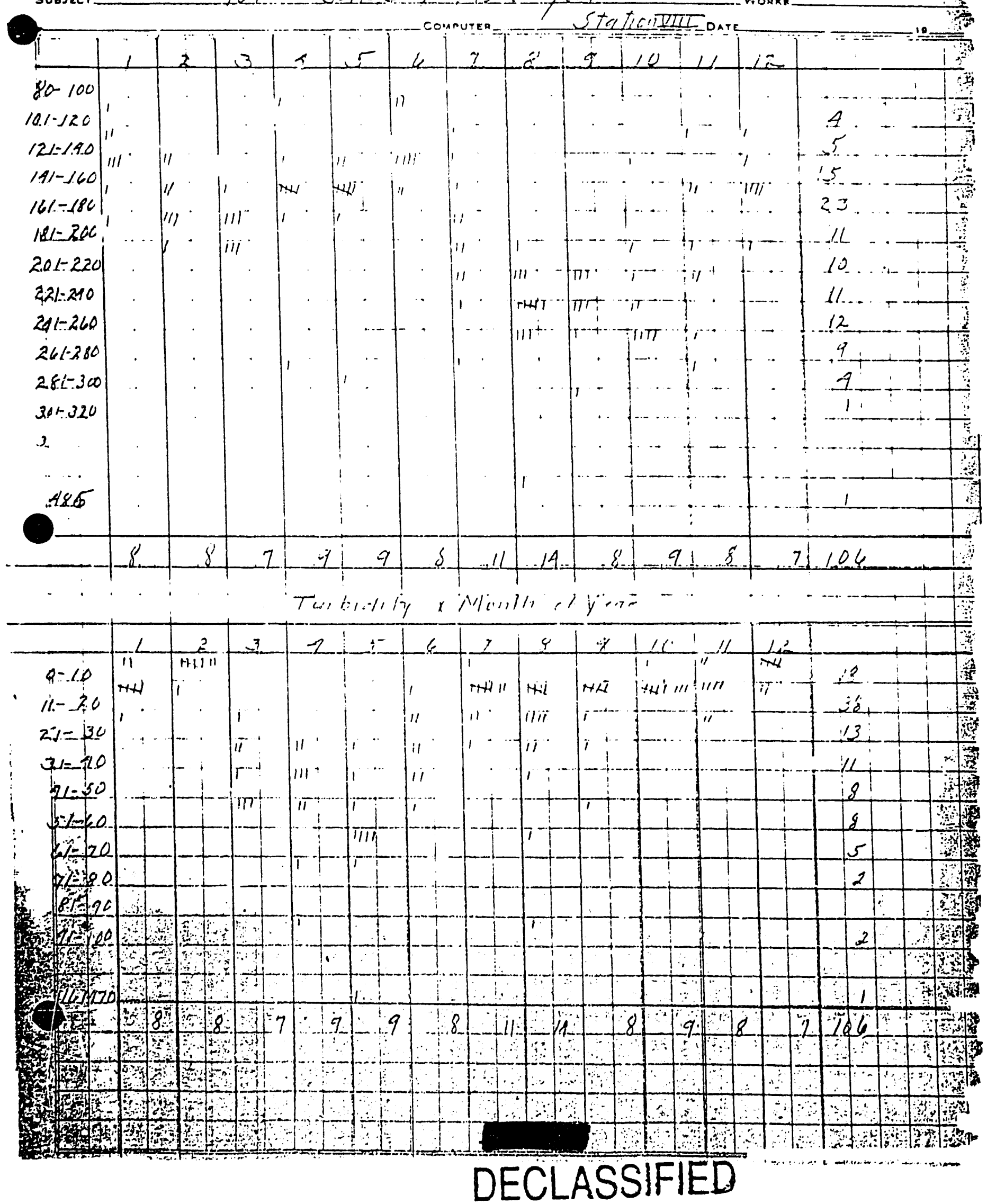


\section{0킼SS $\forall 70 \exists \square$}

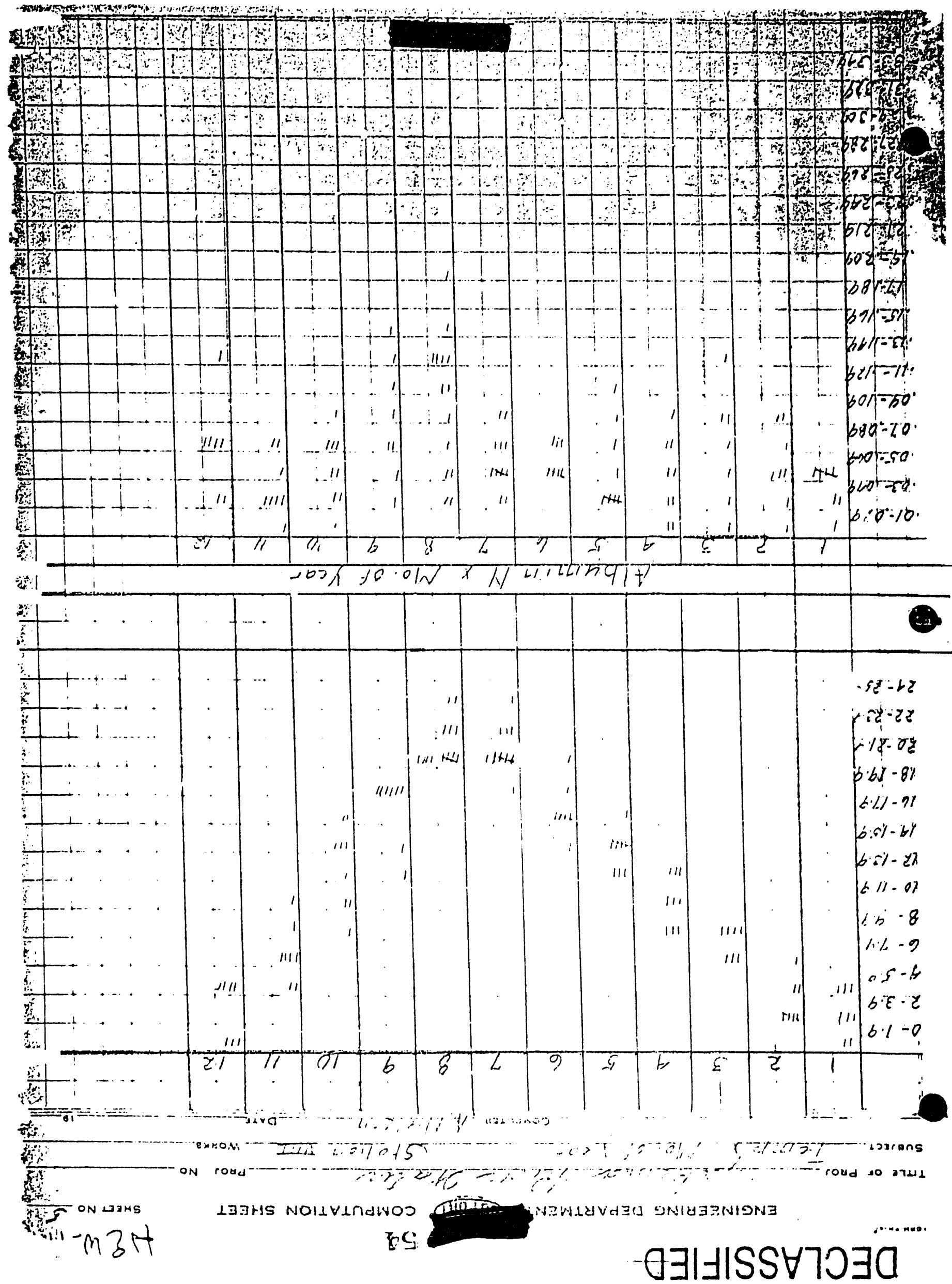



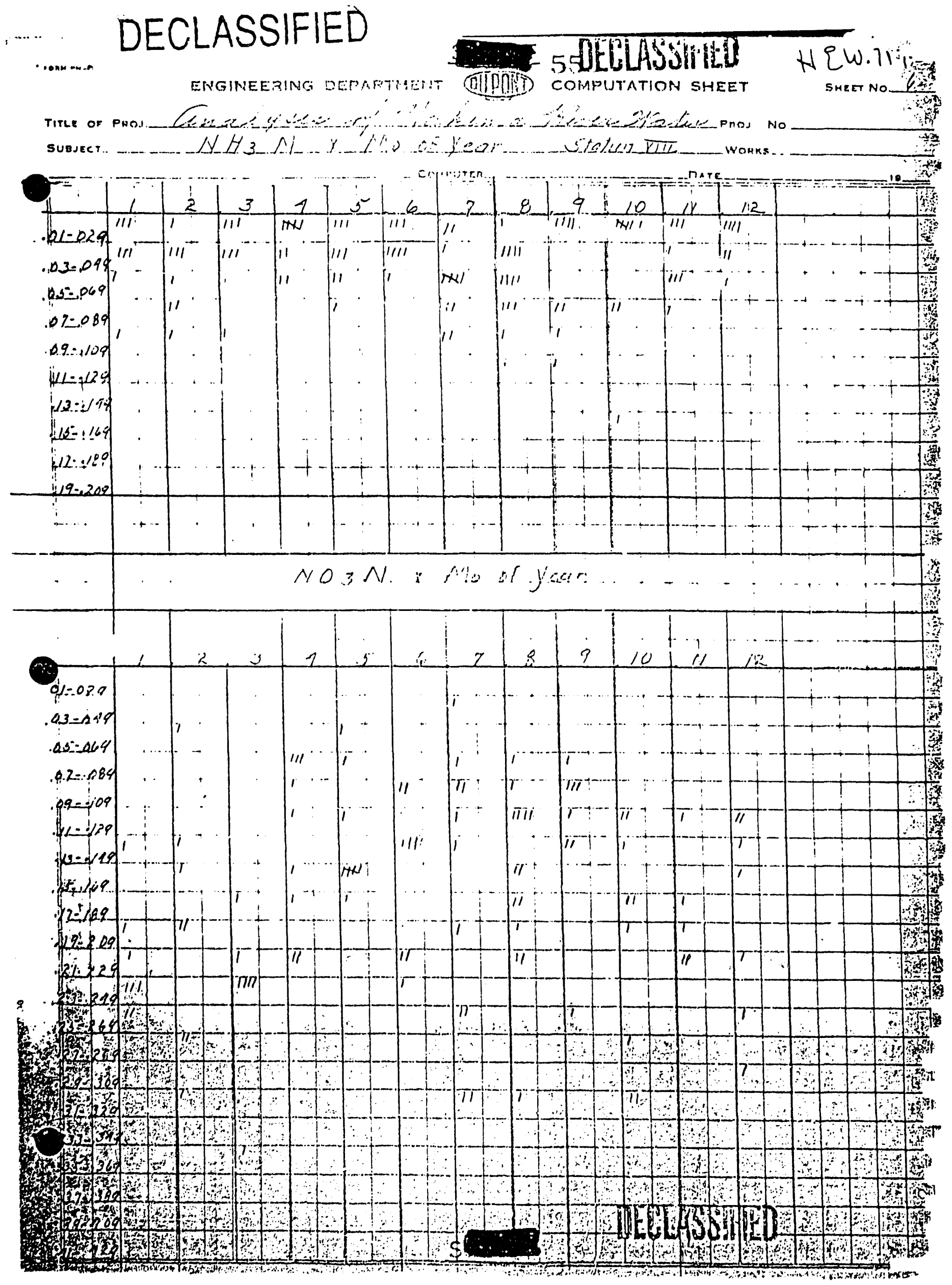

DECLASSIFIED 


\section{व키키S $\forall 70 \exists \square$}

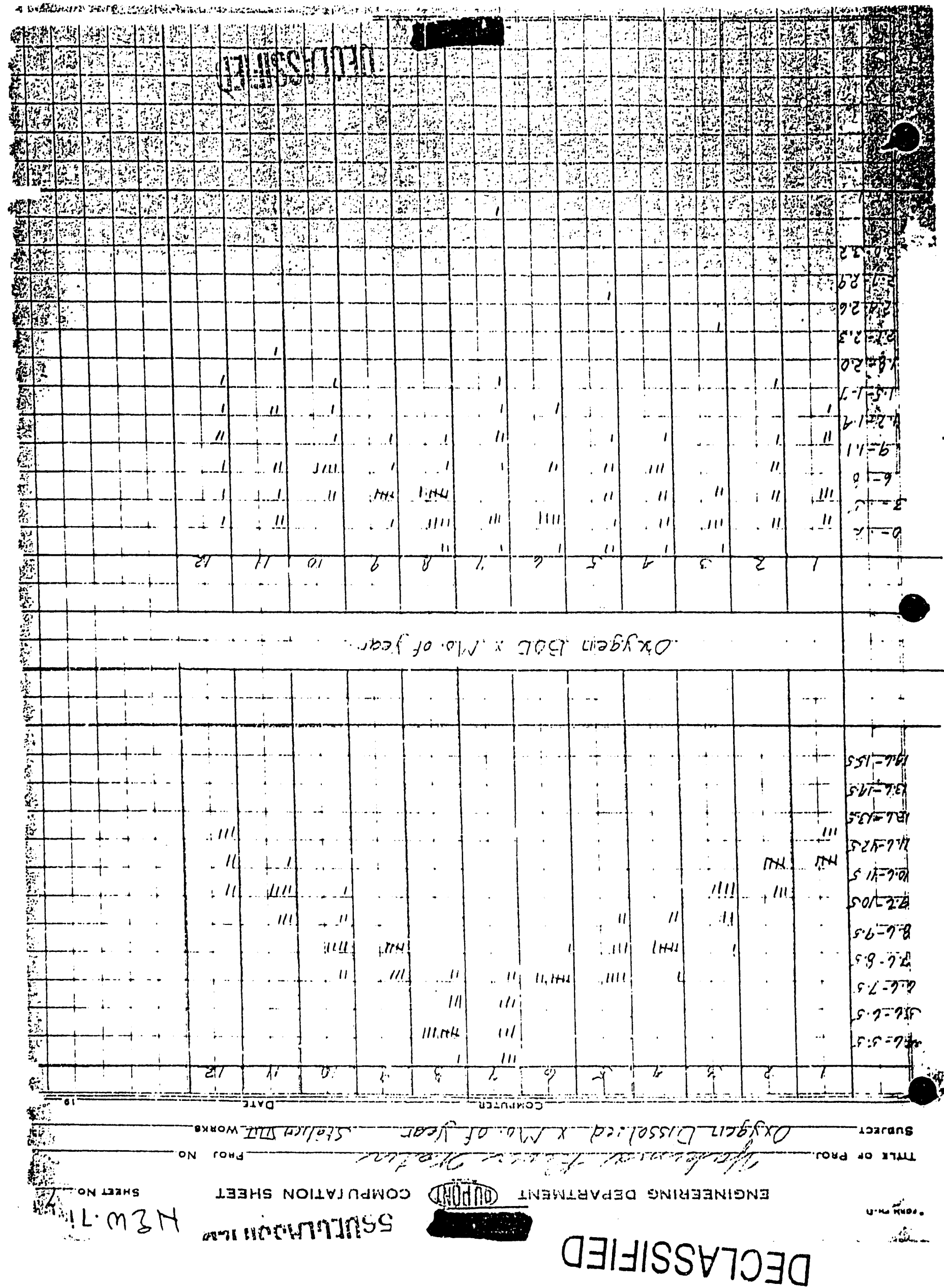



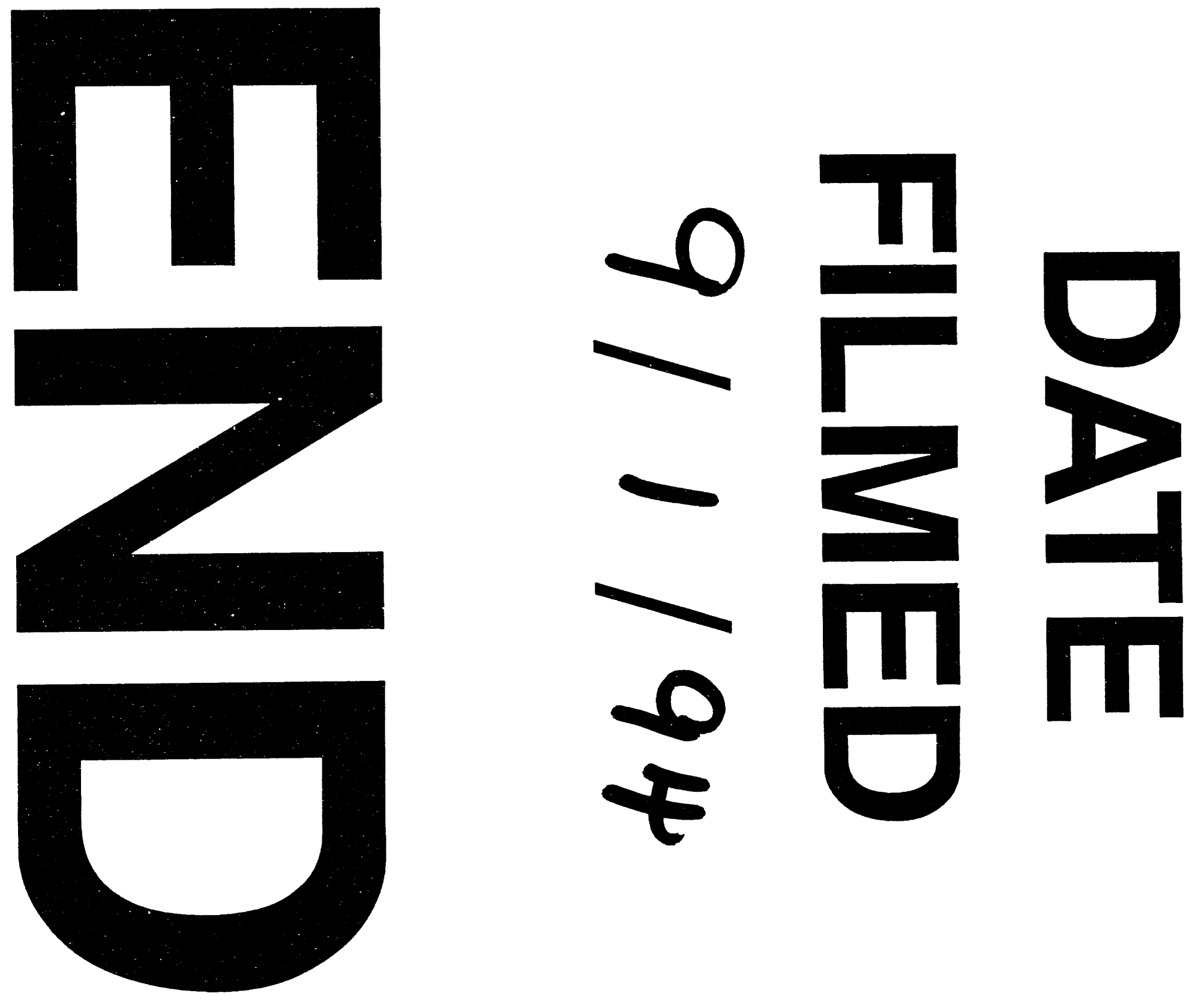


$$
-
$$

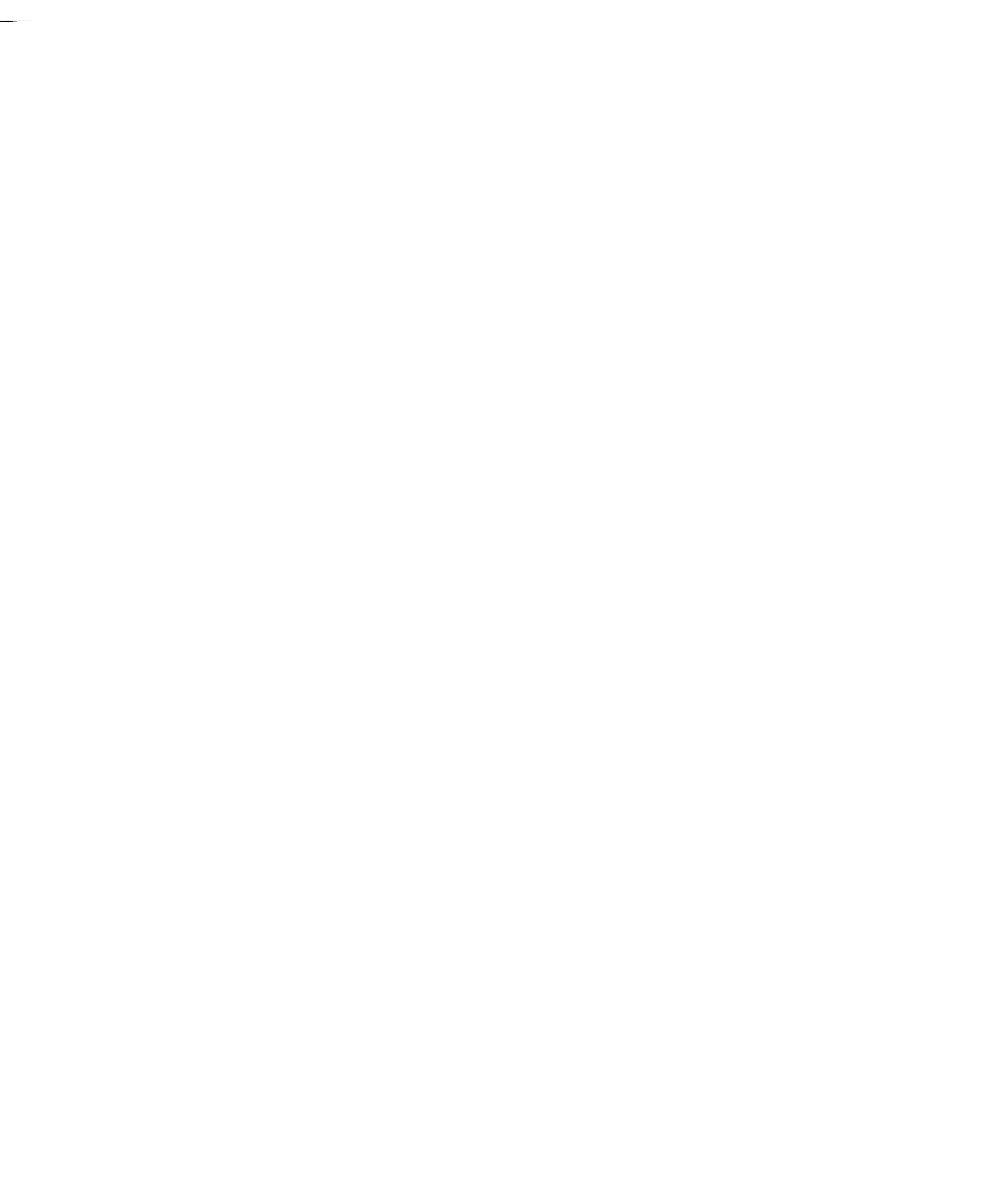

